\title{
HALL EFFECT MODELING IN FEM SIMULATORS AND COMPARISON TO EXPERIMENTAL RESULTS IN SILICON AND PRINTED SENSORS
}

\author{
A Thesis \\ presented to \\ the Faculty of California Polytechnic State University, \\ San Luis Obispo
}

\author{
In Partial Fulfillment \\ of the Requirements for the Degree \\ Master of Science in Electrical Engineering
}

by

Leonardo Alexander Frem

June 2016 
(C) 2016

Leonardo Alexander Frem

\section{ALL RIGHTS RESERVED}




\section{COMMITTEE MEMBERSHIP}

TITLE:

Hall Effect Modeling in FEM Simulators and Comparison to Experimental Results in Silicon and Printed Sensors

AUTHOR: $\quad$ Leonardo Alexander Frem

DATE SUBMITTED: June 2016

COMMITTEE CHAIR: Tina H. Smilkstein, Ph.D.

Associate Professor of Electrical Engineering

COMMITTEE MEMBER: Jane Zhang, Ph.D.

Professor of Electrical Engineering

COMMITTEE MEMBER: Malcolm G. Keif, Ph.D.

Professor of Graphic Communication 


\section{ABSTRACT}

Hall Effect Modeling in FEM Simulators and Comparison to Experimental Results in Silicon and Printed Sensors

Leonardo Alexander Frem

Finite element method simulation models for thin-film semiconductor-based Hall sensors were developed using secondary data in order to understand their behavior under strong magnetic fields. Given a device geometry and charge carrier density and mobility, the models accurately calculated sensor resistance, Hall voltage under a normally-incident constant magnetic field, and expected offset from a population of Hall devices. The model was successfully matched against data from integrated chip Hall sensors from St. Jude Medical. Additionally, the feasibility of creating Hall effect devices with common carbon ink was explored experimentally. The material properties obtained from testing these ink-based devices through the Van der Pauw method were added to the simulation model to analyze validity of the collected data.

Keywords: Finite element method (FEM) model, Hall sensors, printed electronics. 


\section{ACKNOWLEDGMENTS}

I would like to acknowledge and give special thanks to the people listed below (in no particular order); without their help, this project would not have been possible. Their support was a constant source of motivation which lead to a successful defense of this thesis.

- Frank Wei - SJM Technical Advisor

- Dr. Tina Smilkstein - Committee Chair

- Dr. Malcolm Keif - Committee Member

- Dr. Jane Zhang - Committee Member

- Bryce Beatty - Master's Candidate, Printed Electronics and Functional Imaging

- Nikki Bennett - Fiancé

- The Frem and Agundez families 


\section{TABLE OF CONTENTS}

Page

LIST OF TABLES

ix

LIST OF FIGURES

$x i$

CHAPTER

1 Introduction: The Hall Sensor 1

1.1 Basics of Hall Sensor Physics 2

2 COMSOL Multiphysics Simulations 6

2.1 Developing the Magnetoconductivity Model 7

2.2 Validation of the 2D Magnetoconductivity Model with COMSOL 11

2.3 Simulation of SJM's Sensor Ideal Model 14

$\begin{array}{ll}\text { 2.3.1 Global Parameters } & 17\end{array}$

$\begin{array}{ll}\text { 2.3.2 Geometry } & 17\end{array}$

$\begin{array}{lll}2.3 .3 & \text { Materials } & 18\end{array}$

$\begin{array}{lll}2.3 .4 & \text { Physics } & 18\end{array}$

$\begin{array}{lll}2.3 .5 & \text { Mesh } & 20\end{array}$

$\begin{array}{lll}2.3 .6 & \text { Study } & 21\end{array}$

$\begin{array}{lll}2.3 .7 & \text { Results } & 22\end{array}$

2.4 Gaussian Junction Depth 24

2.4.1 Simulation Setup $\quad 26$

2.4.2 Simulation Results and Discussion 28

2.5 Ramping Gaussian Junction Depth 30

2.5.1 Simulation Setup 31 
2.5.2 Simulation Results and Discussion

2.6 Hall Effect Temperature Dependence Progress 34

2.6.1 Simulation Setup and Results 36

$\begin{array}{lll}2.7 & \text { Future Work } & 39\end{array}$

3 Conductive Ink Sensor $\quad 41$

3.1 Introduction to Printed Electronics 41

3.2 Initial Printed Hall Sensor Considerations 43

$\begin{array}{lll}3.3 & \text { First Printed Sensors } & 45\end{array}$

3.4 Helmholtz Coils $\quad 46$

3.5 Van der Pauw Method $\quad 49$

3.5.1 Resistivity and Sheet Resistance $\quad 50$

3.5.2 Hall Voltage, Sheet Density and Majority Carrier Mobility 52

3.5.3 Analysis of the Van der Pauw Method 54

3.6 Neodymium Magnet Pair Test 57

$\begin{array}{lll}\text { 3.6.1 Test Fixture Setup } & 58\end{array}$

$\begin{array}{lll}\text { 3.6.2 Testing and Results } & 60\end{array}$

$\begin{array}{ll}\text { 3.6.2.1 Preliminary Testing } & 60\end{array}$

3.6.2.2 Offset Reduction via Oversized Contacts 62

3.6.2.3 Sensors with Silver Contacts 64

3.6.2.4 Results from the Van der Pauw Method Revisited 65

$\begin{array}{lll}3.7 & \text { Future Work } & 67\end{array}$

4 Semiconductor Hall Sensor $\quad 70$

$\begin{array}{lll}\text { 4.1 Motivation and Current Progress } & 70\end{array}$ 
4.2 Future Work

5 Summary of Results $\quad 74$

$\begin{array}{lr}\text { REFERENCES } & 80\end{array}$

APPENDIX: SEMICONDUCTOR HALL DEVICE FABRICATION PROCEDURES

85 


\section{LIST OF TABLES}

Table

Table 2-2. Histogram bin frequency distribution with respective offset values.

Table 2-3. Offset statistical summary of $\mathrm{TtoB}$ and LtoR ramping functions with respect to Gaussian random noise standard deviation and ramp slope.

Table 2-4. Effects on sensor offset from changes in standard deviation and ramp from $1 \%$ to $10 \%$.

Table 2-5. Magnetoconductivity models used for COMSOL simulations.

Table 3-1. Approximate graphite material properties [27], [28], [29, p. 61]. ${ }^{\dagger}$ Sheet resistance was obtained from manufacturer's datasheet [26].

Table 3-2. Resistance measurements using the Van der Pauw method. The first column, Configuration, refers to the terminals of the Hall sensor. For example, configuration 12,34 has a current being sinked through terminal 1 and 2, while measuring voltages from 3 and 4 . In all cases, the current applied was $1 \mathrm{~mA}$. The last column refers to the calculated percent difference between each reciprocal terminal configurations (i.e. 12,34 and 34,12 are a pair of reicprocal terminal configurations).

Table 3-3. Hall voltage measurements under the influence of magnetic fields. The first column refers to the terminals which were used for the 
biasing current. The second and third column refer to positive and negative fields applied, respectively. The last column represents the difference between columns two and three, which represents the resulting Hall voltage for that particular configuration.

Table 3-4. Estimated and experimental results for the Van der Pauw method, assuming the ink approximately behaves like graphite [29]. ${ }^{\dagger}$ Sheet resistance was obtained from manufacturer's datasheet [26].

Table 3-5. Hall voltage data for the Van der Pauw method. The calculated Hall voltage from this table is $98 \mu \mathrm{V}$ at $527 \mathrm{G}$.

Table 3-6. Comparison of Van der Pauw method results from using the Helmholtz coil pair and the neodymium magnets with silver contacts on the sensors. $15 \mu \mathrm{m}$ is the assumed PET sensor thickness.

Table 5-1. Histogram bin frequency distribution with respective offset values.

Table 5-2. Comparison of Van der Pauw method results from using the Helmholtz coil pair and the neodymium magnets with silver contacts on the sensors. $15 \mu \mathrm{m}$ is the assumed PET sensor thickness. 


\section{LIST OF FIGURES}

Figure

Figure 1-1. Schematic representing an n-type Hall effect system. I is the injected constant current. $B$ is the constant magnetic field, perpendicular to the surface of the conductor. The interaction between the current and magnetic field will yield an accumulation of charge carriers transverse to the current direction and hence a Hall voltage $V_{H} . F_{m}$ and $F_{e}$ indicate the magnetic force and electric field force, respectively [2].

Figure 2-1. Cross-shaped Hall device used by Sun and Kosel [7].

Figure 2-2. Sun and Kosel's potential distribution inside the Hall device (a) without and (b) with a $5 \mathrm{~T}$ magnetic field. The color bar indicates the strength of the voltage surface potential in volts. The current flows from left to right [7].

Figure 2-3. Sun and Kosel's Hall voltage as a function of the magnetic field calculated analytically and by FEM. The inset shows the error between the results from these two methods [7].

Figure 2-4. COMSOL Hall device geometry. Units in $\mu \mathrm{m}$.

Figure 2-5. Hall device surface potential with $5 \mathrm{~T}$ magnetic field.

Figure 2-6. Hall voltage obtained via COMSOL FEM simulation.

Figure 2-7. Example of COMSOL's Model Builder window.

Figure 2-8. Resulting Hall device geometry used in COMSOL. Image has been blurred due to NDA. 
Figure 2-9. COMSOL Physics applied to Hall device geometry. The purple area represents where Current Conservation is present.

Figure 2-10. (a) Mesh built for entire geometry. (b) Close-up of bottom-left corner of mesh to show details. The mesh consists of 20448 domain elements and 624 boundary elements.

Figure 2-11. Sensor resistance vs. electron mobility with different electron density values.

Figure 2-12. Sensor Hall voltage at constant 1.5T magnetic field vs. electron mobility with different electron density values. Target value is reached with electron mobility value of $0.0432 \mathrm{~m}^{2} /(\mathrm{V} \mathrm{s})$. Note that all electron density lines overlap, hence proving that this magnetoconductivity model disregards electron density for Hall voltage calculation.

Figure 2-13. Sensor offset with respect to varying electron density and mobility values. The Sensor Offset axis is not a mistake. The extremely small variation in offset is most likely due to symmetrical mesh element misalignment.

Figure 2-14. Output of Random function. Notice that the random distribution is centered around 1. The different planes or slices shown represent the distinct spatial variations introduced via a random seed, which represents the 3rd argument for this function.

Figure 2-15. Example of conductivity model affected by discretized ion implantation imperfections. random_num(167) $=0.66667$ denotes the 
random seed used. A different seed produces a different conductivity distribution from the one seen above. The color bar on the right represents the conductivity value in $\mathrm{S} / \mathrm{m}$.

Figure 2-16. Histogram distribution for sensor offset values. The numbers in the $\mathrm{x}$-axis denote bin numbers as per Table 2-2.

Figure 2-17. Carrier concentration profile [16, Fig. 2] showing the advantage of using a $7^{\circ}$ tilt in comparison to no tilt.

Figure 2-18. (a) TtoB ramping conductivity example. (b) LtoR ramping conductivity example. random_num indicates the seed used for the Gaussian noise. The Gaussian has a standard deviation of $10 \%$. The ramp function has a slop of $10 \%$ of the original conductivity.

Figure 2-19. Summary of Hall voltage vs. temperature simulations. Equations displayed represent trend lines calculated by Excel. BetaT_Vhall was the only simulation that approached our expected results.

Figure 2-20. Summary of results for sensor resistance vs. temperature. Most magnetoconductivity models follow the increasing trend of mu_eT_global, which exception of NoTempCo and BettaT.

Figure 3-1. Comparison between printed and conventional electronics technologies [23].

Figure 3-2. Printed electronics expected market cost (2016-2018) [24].

Figure 3-3. Examples of sensor printed in uncoated cardboard. Left:

9x9 mm sensor. Right: $18 \times 18 \mathrm{~mm}$ sensor. 
Figure 3-4. Helmholtz coils used during experiments. Each coil has 400 turns, can withstand a maximum of $1 \mathrm{~A}$ of current, and have an average coil diameter of $155 \mathrm{~mm}$. The outer diameter measures 165 $\mathrm{mm}$ and the inner diameter $145 \mathrm{~mm}$. Note that these coils are hobbyist grade [31].

Figure 3-5. (a) Cross-section of Helmholtz coil pair and the resulting magnetic field lines with matched current direction. (b) Contours showing the uniformity of the magnitude of the magnetic field near the Helmholtz coil pair. Inside the central "octopus", the field is within $1 \%$ of its central value B0. The eight contours are for field magnitudes of 0.5 B0, 0.8 B0, 0.9 B0, 0.95 B0, 0.99 B0, 1.01 B0, 1.05 B0, and $1.1 \mathrm{~B} 0[32]$.

Figure 3-6. COMSOL Helmholtz coil pair geometry used. Average coil diameter of $155 \mathrm{~mm}$. The outer diameter measures $165 \mathrm{~mm}$ and the inner diameter $145 \mathrm{~mm}$. To maximize the uniformity of magnetic field, the coils are half a radius away from each other, i.e. $38.75 \mathrm{~mm}$.

Figure 3-7. COMSOL Helmholtz coil pair simulation using parameters from the coils used for experiments. The approximate field strength in the middle of the coils is $150 \mu \mathrm{T}$.

Figure 3-8. Diagram showing the terminal configuration numbering convention for Van der Pauw method method and calculations. 
Figure 3-9. (a) Helmholtz coil test setup. The triple power supply provides power to each of the coils and the biasing current for the sensor. (b) Sensor placement on the Helmholtz coil.

Figure 3-10. Offset sampling over time. The sample number corresponds to the time the offset was sampled, i.e. sample 1 was the first offset measurement and 15 the last.

Figure 3-11. Close-up from one of the sensors printed on a glass substrate. The scratches come from probing and handling the sensor. Note that the scratches did not completely remove the ink from the glass substrate.

Figure 3-12. Magnet pair test setup. If the measurement required a magnetic field, the magnets were placed 5 or $7 \mathrm{~cm}$ away from the sensor, depending on desired field strength. If no magnetic field was required, then the magnets were removed. The sensor is connected to horizontal current biasing and vertical voltage measurement or vice-versa. The $\mathrm{G}$ represents the Gaussmeter placement.

Figure 3-13. Magnet pair test fixture setup. The silver cubes are the magnets. The device in the middle is the constructed Gaussmeter. The white cutouts serve as the $5 \mathrm{~cm}$ markers and also prevent the magnets from collapsing onto each other when placed at this spot. Not shown is the sensor base, which fits in the middle slot.

Figure 3-14. Magnetic field lines visualization for an attracting pair of magnets [38]. 
Figure 3-15. Preliminary test for $18 \mathrm{~mm}$ glass sensor biased at $1 \mathrm{~mA}$.

The highly linear responses showed promising results for future indepth tests.

Figure 3-16. Preliminary test 2 . Results from holding the biasing current at $8 \mathrm{~mA}$ and modifying the magnetic field strength by modifying the positioning of both cube magnets. The sensor yielded completely different offset voltage when compared to Figure 3-15.

Similarly, the voltage/field slope flipped polarity.

Figure 3-17. Picture showing damaged sensors from metal contact bonding attempts.

Figure 3-18. Sensor on PET film with silver contacts. This device was the most effective at eliminating the offset drift.

Figure 3-19. $18 \mathrm{~mm}$ sensor with silver contacts tested at $100 \mathrm{mV}$ bias.

Figure 3-20. $9 \mathrm{~mm}$ sensor with silver contacts tested at $5 \mathrm{~mA}$ bias.

65

Figure 3-21. Simulation based on results obtained from the neodymium Van der Pauw method test.

Figure 3-22. Block diagram of CMOS integrated linear Hall sensory microsystem [39].

Figure 4-1. Cross-section of Hall sensor device in silicon chip.

Figure 4-2. Individual chip with 8 distinct Hall devices. Mask \#1 (white) is used for dopant diffusion. Mask \#2 (blue) helps setting the metal contacts, and Mask \#3 (magenta) connects the metal contacts to vias 
towards the end of each chip for ease of access to each sensor. Nand p-type refer to the doping polarity in that specific region.

Figure 4-3. Dimensions of all designed Hall devices. d8 denotes the separation between the edge of the octagon and its hole. d10 denotes the horizontal and vertical separation between the sensor and its contact pads. d11 denotes the size of the internal square.

Figure 4-4. Layered exposure masks for a single wafer. Several Hall devices were designed to test performance of different geometries. compared to Figure 3-15. Similarly, the voltage/field slope flipped polarity.

Figure 5-1. Sun and Kosel's Hall voltage as a function of the magnetic field calculated analytically and by FEM. The inset shows the error between the results from these two methods [7].

Figure 5-2. Hall voltage obtained via COMSOL FEM simulation.

Figure 5-3. Sensor resistance vs. electron mobility with different electron density values.

Figure 5-4. Sensor Hall voltage at constant 1.5T magnetic field vs. electron mobility with different electron density values. Target value is reached with electron mobility value of $0.0432 \mathrm{~m}^{2} /(\mathrm{V} \mathrm{s})$. Note that all electron density lines overlap, hence proving that this magnetoconductivity model disregards electron density for Hall voltage calculation. 
Figure 5-5. Example of conductivity model affected by discretized ion implantation imperfections. random_num(167) $=0.66667$ denotes the random seed used. A different seed produces a different conductivity distribution from the one seen above. The color bar on the right represents the conductivity value in $\mathrm{S} / \mathrm{m}$.

Figure 5-6. Histogram distribution for sensor offset values. The numbers in the $\mathrm{x}$-axis denote bin numbers as per Table 2-2.

Figure A-1. P-type silicon wafer.

Figure A-2. Oxide layer grown on silicon wafer. 86

Figure A-3. Positive photoresist applied to wafer. 86

Figure A-4. Exposing photoresist to UV light with a mask aligner. 87

$\begin{array}{ll}\text { Figure A-5. Positive photoresist developed and washed away. } & 87\end{array}$

Figure A-6. Selective etching of oxide film. 88

Figure A-7. Photoresist completely stripped from wafer. 88

Figure A-8. n-type dopant on wafer. $\quad 88$

Figure A-9. Diffusion of n-type dopant on wafer by pre-deposition. 90

Figure A-10. Clean wafer with n- and p-type dopant. 90

Figure A-11. Diffusion of n-type dopant on wafer by pre-deposition. 90

Figure A-12. Diffusion of n-type dopant on wafer by pre-deposition. 91

Figure A-13. Diffusion of n-type dopant on wafer by pre-deposition. 91

Figure A-14. Diffusion of n-type dopant on wafer by pre-deposition. 91 


\section{Introduction: The Hall Sensor}

Hall effect sensors, which are based on magnetic phenomena, are one of the most commonly used sensing technologies today. These sensors are primarily employed as current sensors by detecting the magnetic field emanating from a specific conductor and serve many other low-power applications. One of St. Jude Medical's (SJM) pacemakers involves a Hall-effect sensor for the purpose of sensing strong magnetic fields like the ones generated when performing magnetic resonance imaging (MRI). Pacemakers typically have leads connected to the heart, and, in the presence of a magnetic field, they might couple to the field, which causes the leads to start heating up. The goal of this Hall sensor is to detect these magnetic fields and activate circuitry to prevent the leads from affecting the pacemaker's behavior and hence affecting the patient's health. Patients can someday rely on this sensor to prevent a magnetic field from causing serious harm or even death to the patient. Hence, it is imperative to fully understand the behavior of sensor under specific conditions to avoid any potential harm to patients with pacemakers. According to Reuters, "In 1993, there were about 121,300 pacemaker implantations in the U.S. By 2009 , that number was 188,700 - a 56 percent increase [1]." As time progresses, it seems that more people in the U.S. need pacemakers, and hence the importance of this research.

SJM wants a fitting model for their current Hall sensor so that they can do more exploration in a simulated environment and thus make faster, more accurate and more complete design decisions before manufacturing devices. 
Having a working detailed model allows SJM to consider all parameters that might influence the behavior of the Hall sensor. This project provides SJM with a reliable, and adjustable, 2D model of their Hall sensor to assist in SJM's analysis. The 2D model simulates the constant electromagnetic field acting on the sensor and predicts its electrical behavior given the sensor parameters and material properties, such as electron mobility, charge carrier density, sensor biasing voltage or current and even temperature. A 2D model was preferred over a 3D model due to computation time, and ease of use. Additionally, to build-up to a more complex model, it is typically better to start with a simpler model.

\subsection{Basics of Hall Sensor Physics}

The Hall effect works under the physics of electric current in conductors. Electric current typically consists of movement of charge carriers, which can be negative or positive charge, electrons or holes, respectively. In the presence of a magnetic field, these charge carriers experience a force commonly known as Lorentz force. In the absence of such magnetic field, charge carriers follow the path of least resistance, typically a straight line in a conductor. However, if a magnetic field is perpendicular to the direction of the current flow, the charge carriers are deflected from their original straight path, causing an asymmetric accumulation of charge on

the edges of the conductor. The asymmetric accumulation of charges gives rise to a voltage potential, better known as Hall voltage. 
The interaction between charges and the magnetic Lorentz force results in an uneven or anisotropic conductivity. The force acting on a single charge carrier can be expressed as

$$
\boldsymbol{F}=q(\boldsymbol{E}+(\boldsymbol{v} \times \boldsymbol{B}))
$$

where $\boldsymbol{F}$ is the force vector deflecting the charged carrier, $q$ is the charge of the carrier, $\boldsymbol{E}$ is the electric field vector, $\boldsymbol{v}$ is the instantaneous drift velocity vector of the carrier and $\boldsymbol{B}$ is the magnetic field vector. Drift velocity represents the flow velocity of a particle due to an electric field. The term $q E$ is better known as electric field force, while $q \boldsymbol{v} \times \boldsymbol{B}$ represents the magnetic force $\boldsymbol{F}_{\boldsymbol{m}}$.

In a solid conductor, the current can be expressed in terms of the drift velocity as

$$
\boldsymbol{I}=\boldsymbol{j} \times A=n q \boldsymbol{v} w d
$$

where $\boldsymbol{j}$ is the current density and $n$ is the number of charge carriers. $A$ denotes the cross-section area of the conductor, which in turn can be represented as $A=$ $w d$, where $w$ and $d$ are the width and thickness of the conductor, respectively. The drift velocity $\boldsymbol{v}$ can then be rewritten as

$$
v=\frac{\mathbf{I}}{n q w d}
$$




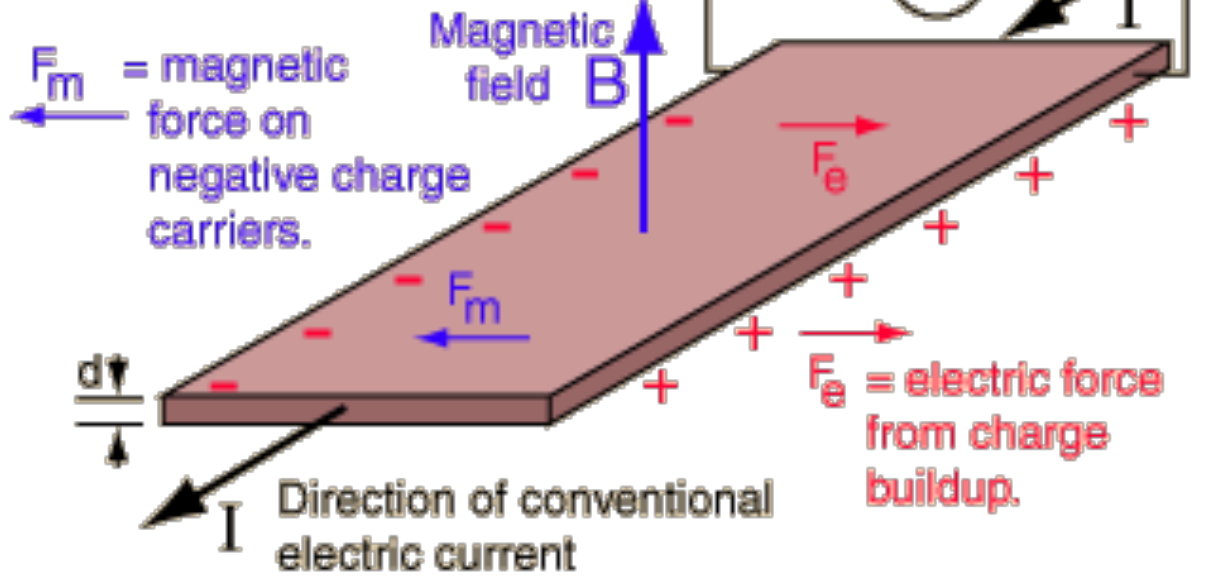

Figure 1-1. Schematic representing an n-type Hall effect system. $I$ is the injected constant current. $B$ is the constant magnetic field, perpendicular to the surface of the conductor. The interaction between the current and magnetic field will yield an accumulation of charge carriers transverse to the current direction and hence a Hall voltage $V_{H} . F_{m}$ and $F_{e}$ indicate the magnetic force and electric field force, respectively [2].

The deflection of moving charge carriers due to the magnetic field causes accumulation of charges of opposite sign at the edges of the conductor, creating the Hall field $E_{H}$. When magnetic and electric field forces are equivalent $\left(F_{m}=F_{e}\right)$, equilibrium is achieved as described by

$$
q \boldsymbol{v} \times \boldsymbol{B}=\frac{V_{H} q}{d}
$$

$V_{H}$ represents the Hall voltage caused by the electric field of the accumulated charge carriers. Solving for the Hall voltage, and using the current in terms of drift velocity equation

$$
V_{H}=\frac{I B}{n q d}
$$


Considering that n-type semiconductors are more commonly used, and the respective charge carriers are negative (electrons), the previous equation can be modified to

$$
V_{H}=-\frac{I B}{n e d}
$$

where e represents the elementary electric charge for an electron $1.602 \times 10^{19} \mathrm{C}$. 


\section{COMSOL Multiphysics Simulations}

The Hall voltage equation (6) presented in the previous chapter represents a very simple model for a very complex problem given that Hall sensors can vary in geometric shape and materials used for fabrication. Additionally, semiconductors change behavior on external factors such as stress and temperature [3]. Finite element method (FEM), also known as finite element analysis (FEA), provides the ease to break down a complex problem into a simpler one. To do so, FEM presents a method for numerical solution of diverse fields that involve boundary value problems for partial differential equations. Some of these involve elastic, thermal, fluid flow, and electrostatic problems, amongst other types. FEM divides a problem or structure into smaller, simpler parts, called finite elements. The FEM connects these elements together, and the field quantity, depending on the relevant physics for the problem in question, becomes interpolated over the structure in a piecewise fashion, which gives rise to a set of algebraic equations at the nodes of the finite elements [4], [5].

COMSOL Multiphysics is an FEM simulation software. COMSOL provides users with diverse modules to model and simulate any physics-based system, including electrostatics problems, such as determining an adequate model for the Hall sensor. Specifically, COMSOL's AC/DC module can simulate static electric and magnetic fields [6]. 


\subsection{Developing the Magnetoconductivity Model}

Although COMSOL has the capability of simulating 3D objects, performing a single simulation can take a long time, especially with computers with lesser processing capabilities. Thus, making a 2D model of SJM's Hall sensor a good start. That being said, COMSOL does not have a direct method of including an even magnetic field in a 2D domain. However, COMSOL allows material properties, such as electron mobility and density, to vary spatially [6], and as previously mentioned, the interaction between charges and the magnetic Lorentz force results in a spatially uneven or anisotropic conductivity in a Hall sensor. Therefore, the magnetic field and its effect on the Hall sensor can still be modeled with this software using an adequate magnetoconductivity model. Sun and Kosel [7] derivation of the magnetoconductivity model is explained next.

The conductivity $\sigma$ of an isotropic and homogeneous conductor is given by

$$
\sigma \equiv \frac{1}{\rho}=\left(n_{e} \mu_{e}+n_{h} \mu_{h}\right) q
$$

where $\rho$ represents the resistivity, $n_{e}$ and $n_{h}$ represent electron and hole densities, $\mu_{e}$ and $\mu_{h}$ represent electron and hole mobility, respectively. The conductivity tensor of an isotropic conductor in 3D space can be represented as

$$
\boldsymbol{\sigma}=\left(n_{e} \mu_{e}+n_{h} \mu_{h}\right) q\left[\begin{array}{lll}
1 & 0 & 0 \\
0 & 1 & 0 \\
0 & 0 & 1
\end{array}\right]
$$

As previously noted, Hall devices are commonly made from n-type semiconductors because the electron mobility is much greater than that of holes. Hence, the above equation simplifies further to 


$$
\frac{1}{\rho}=n_{e} \mu_{e} q
$$

To describe conductivity dependent on magnetic fields, direct integration of the Boltzmann equation $[8, p .109]$ yields the following formula for current density

$$
\boldsymbol{j}=\boldsymbol{\sigma}_{\mathbf{0}}\left(\boldsymbol{E}+\frac{\boldsymbol{j} \times \boldsymbol{H}}{n_{e} e}\right)
$$

where $\sigma_{0}=n_{e} q \mu$ represents the conductivity in an n-type semiconductor in the absence of magnetic field, and $\boldsymbol{\mu}$ represents the electron mobility tensor. For isotropic conductors, the mobility tensor components are identical in all 3 spatial axes

$$
\boldsymbol{\mu}=\mu_{e}\left[\begin{array}{lll}
1 & 0 & 0 \\
0 & 1 & 0 \\
0 & 0 & 1
\end{array}\right]
$$

Via Ohm's law, the current density becomes

$$
\boldsymbol{j}=\boldsymbol{\sigma}(\boldsymbol{H}) \cdot \boldsymbol{E}
$$

Hence, solving for the magnetoconductivity tensor

$$
\boldsymbol{\sigma}(\boldsymbol{H})=\frac{n_{e} e}{\frac{1}{\boldsymbol{\mu}}+\boldsymbol{H}}
$$

where the magnetic field matrix $\boldsymbol{H}$ is defined as

$$
\boldsymbol{H}=\left[\begin{array}{ccc}
0 & -H_{z} & H_{y} \\
H_{z} & 0 & -H_{x} \\
-H_{y} & H_{x} & 0
\end{array}\right]
$$

$H_{x}, H_{y}$ and $H_{z}$ represent magnetic field components in the $x-, y-$, and z-directions, respectively. Considering that magnetic fields perpendicular to Hall sensors dominate in the overall the Hall effect, and that these devices usually consist of 
thin-film structures to maximize the obtained Hall voltage, the 2D magnetoconductivity model can be reduced to

$$
\boldsymbol{\sigma}(\boldsymbol{H})=\frac{\mu_{e} n_{e} q}{1+\left(\mu_{e} H_{z}\right)^{2}}\left[\begin{array}{ccc}
1 & -\mu_{e} H_{z} & 0 \\
\mu_{e} H_{z} & 1 & 0 \\
0 & 0 & 1
\end{array}\right]
$$

Sun and Kosel present this magnetoconductivity and test their model by applying it to the geometry shown below, in Figure 2-1, to analyze the Hall effect via finiteelement modelling [7].

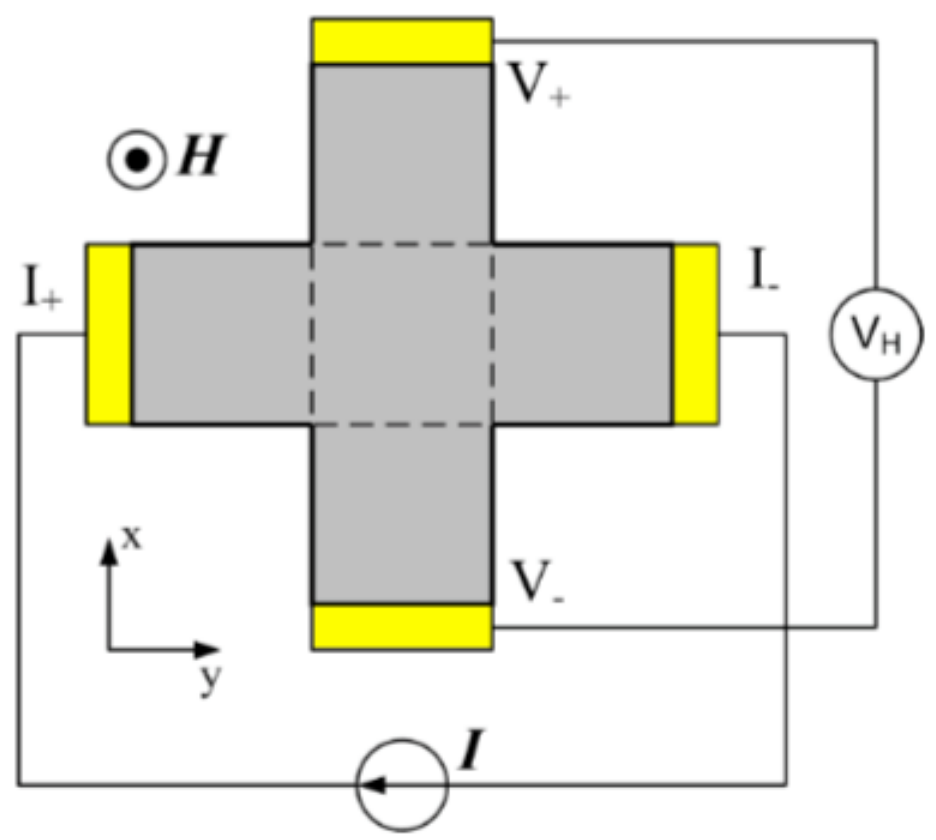

Figure 2-1. Cross-shaped Hall device used by Sun and Kosel [7].

$100 \mu \mathrm{A}$ serves as the biasing current $I . V_{H}$ represents the sensor's measured Hall voltage. $H$ shows the magnetic field flows out of this document. The gray area represents a semiconductor electron mobility of $4.55 m^{\wedge} 2 /(V \cdot s)$, and electron density of $2.55 \times 10^{22} \mathrm{~m}^{-3}$. The yellow area represents gold contacts with electron mobility of $5.3 \times 10^{\wedge} 3 \mathrm{~m}^{\wedge} 2 /(V \cdot s)$ and electron density of $5.9 \times 10^{28} \mathrm{~m}^{-3}$. The Hall voltage equation (5) states that due to the high electron density content in gold the 
Hall effect will not manifest in these gold contacts. The dimensions of said geometry are as follows, the arms are $30 \times 10 \mu \mathrm{m}^{2}$, and the intercross region has an area of $10 \times 10 \mu \mathrm{m}^{2}$. Applying a $5 \mathrm{~T}$ magnetic field yields the potential distribution as shown below, in Figure 2-2. Further, sweeping the magnetic field from $-5 \mathrm{~T}$ to $+5 \mathrm{~T}$ yields the data as seen in Figure 2-3. Sun and Kosel find that the FEM simulation only diverges from the analytical results from 1 to $3 \%$.

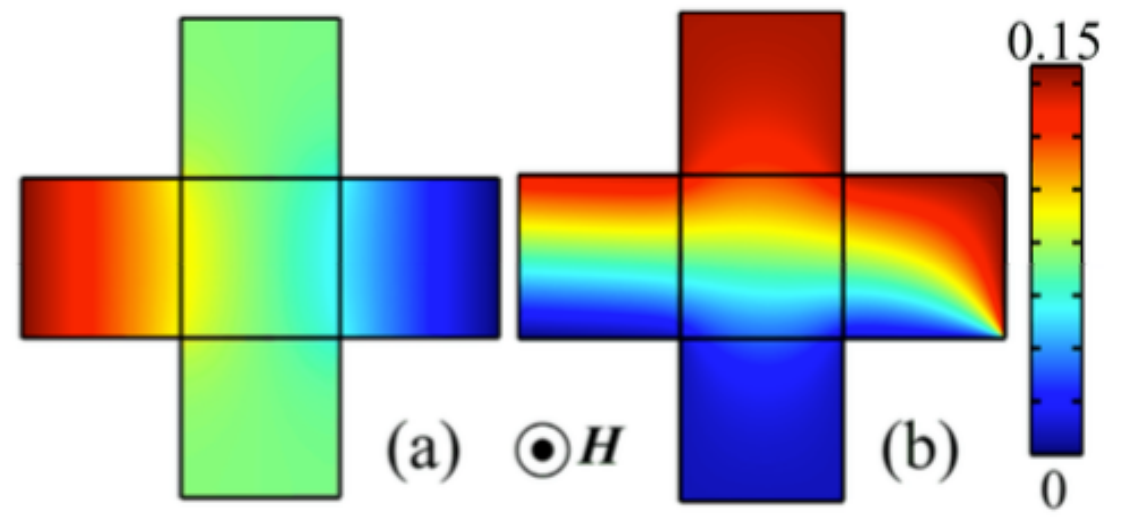

Figure 2-2. Sun and Kosel's potential distribution inside the Hall device (a) without and (b) with a $5 \mathrm{~T}$ magnetic field. The color bar indicates the strength of the voltage surface potential in volts. The current flows from left to right [7]. 


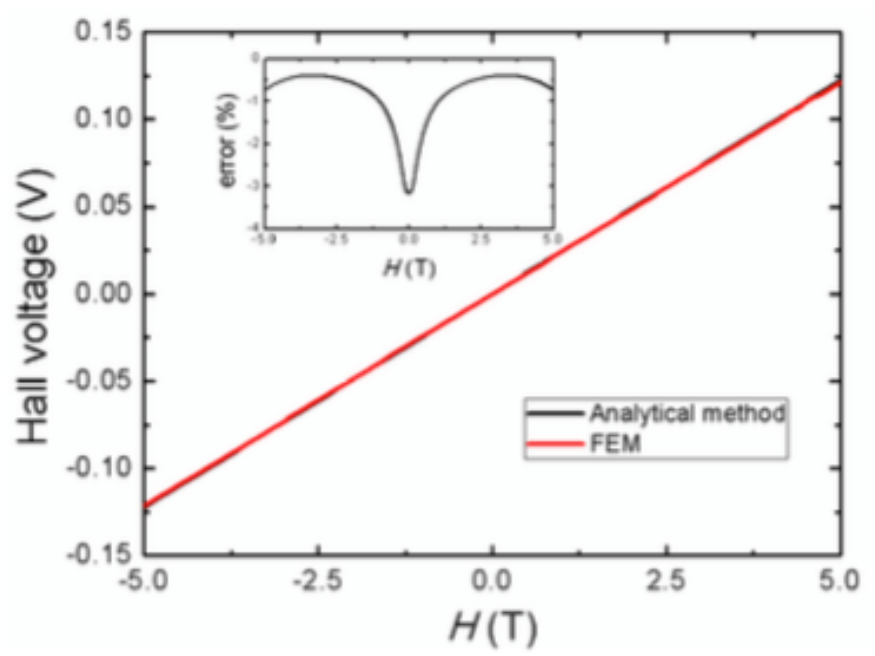

Figure 2-3. Sun and Kosel's Hall voltage as a function of the magnetic field calculated analytically and by FEM. The inset shows the error between the results from these two methods [7].

2.2 Validation of the 2D Magnetoconductivity Model with COMSOL

Since Sun and Kosel supported that their model was accurate via a comparison with analytical calculations. Hence, the first step becomes to show that COMSOL can replicate the above results. The Hall device geometry was replicated via COMSOL, as seen below, in Figure 2-4. For an example with detailed instructions, refer to steps outlined in sections 2.3.1-2.3.7. 


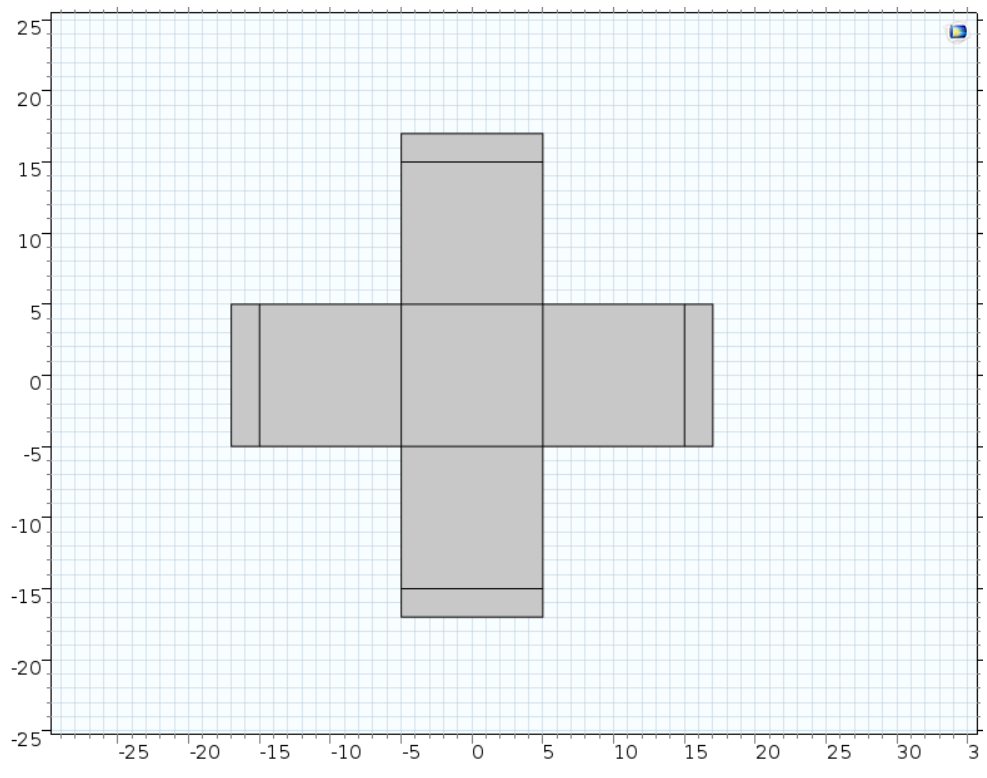

Figure 2-4. COMSOL Hall device geometry. Units in $\mu \mathrm{m}$.

COMSOL needs a device thickness to extrude the object shown above to measure parameters such as resistance. Therefore, thickness of the sensing device was assumed to be $0.1 \mu \mathrm{m}$. Next, the material properties for the sensing and gold contacts were set. The anisotropic magnetoconductivity was set as per (15). Finally, the magnetic field, $\mathrm{H}_{\mathrm{z}}$, was swept from $-5 \mathrm{~T}$ to $+5 \mathrm{~T}$ yielding the results as seen in Figure 2-5 (a) and (b), and Figure 2-6. 


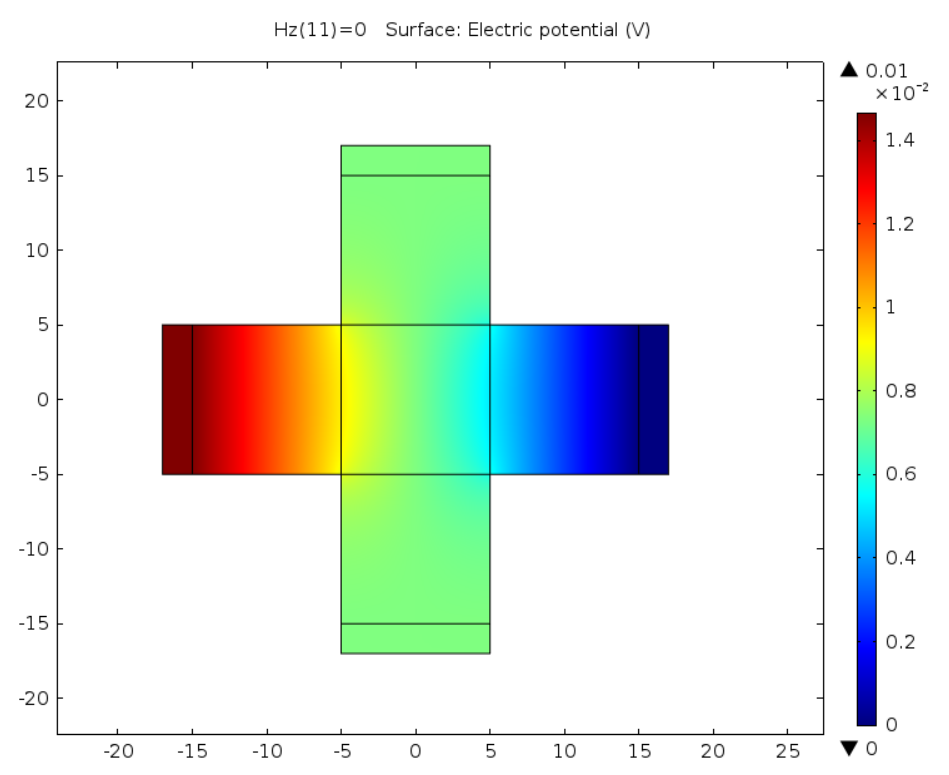

(a)

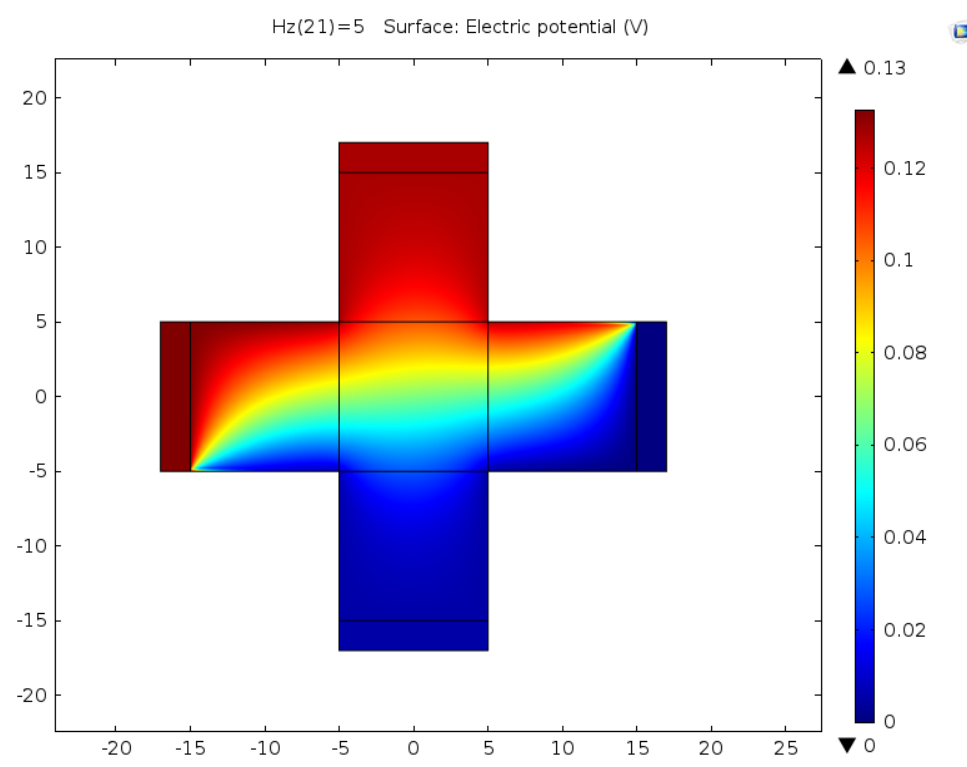

(b)

Figure 2-5. Hall device surface potential with $5 \mathrm{~T}$ magnetic field.

The result shown in Figure 2-5 shows a discrepancy between COMSOL results and those from Sun and Kosel. In Figure 2-2, the surface potential is not evenly distributed across the left arm of the sensor. Further, as previously mentioned, the gold contacts should minimize the Hall effect, and the voltage distribution should 
be as shown on Figure 2-5. Regardless of this minor discrepancy, the Hall voltage as function of the magnetic field was obtained from COMSOL, as seen in Figure 2-6, which matches Sun and Kosel's results. Hence this validates COMSOL as an appropriate FEM software for analyzing the Hall effect on SJM's Hall sensor.

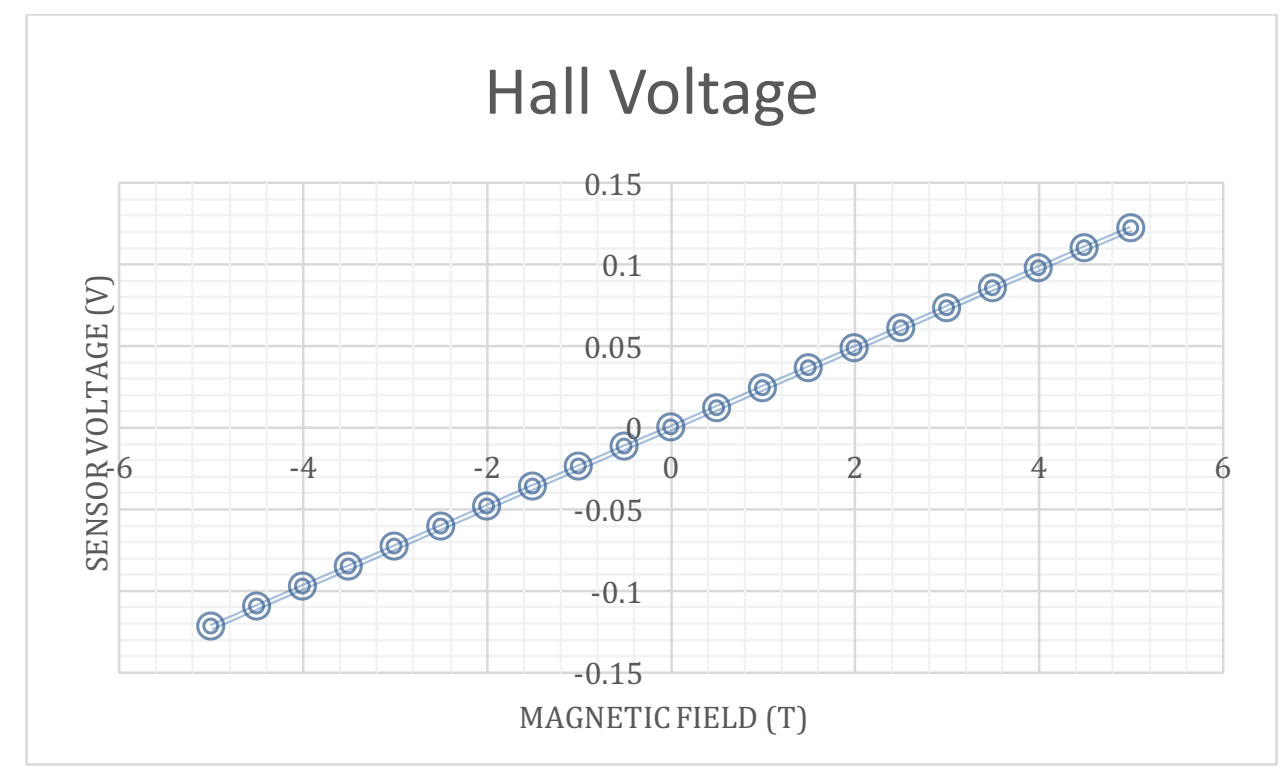

Figure 2-6. Hall voltage obtained via COMSOL FEM simulation.

\subsection{Simulation of SJM's Sensor Ideal Model}

The knowledge of SJM's Hall device's material properties, sensor operating parameters and its behavior greatly assisted in creating a model close to the real sensor. The known sensor parameters are summarized in the table below. Please note that due to a non-disclosure agreement and protection of intellectual properties, all specific values for SJM's Hall sensor have been erased. 
Table 2-1. SJM Hall sensor parameters and operating characteristics. Note that specific values have been deleted due to non-disclosure agreements and protection of intellectual properties.

\begin{tabular}{cccc}
\hline Parameter & Symbol & Value & Unit \\
Electron mobility & $\mu_{e}$ & Estimated at & $\mathrm{m}^{2} /(\mathrm{V} \mathrm{s})$ \\
Electron density & $n_{e}$ & Estimated at & $\mathrm{cm}^{-3}$ \\
Sensor length & $l$ & $\mu \mathrm{m}$ \\
Sensor width & $w$ & $\mu \mathrm{m}$ \\
Sensor thickness & $d$ & & $\mu \mathrm{m}$ \\
Sensor resistance & $R_{\text {Hall }}$ & & $\Omega$ \\
Temperature coefficient & $\alpha$ & & $\%{ }^{\circ} \mathrm{C}$ \\
Biasing voltage & $V_{\text {bias }}$ & & $\mathrm{V}$ \\
Offset voltage & $V_{0}$ & Estimated at & $\mathrm{mV}$ \\
Hall voltage at 1.5 $\mathrm{mT}$ & $V_{\text {Hall }}$ & Estimated at & $\mu \mathrm{V}$ \\
\hline
\end{tabular}

The resistance is measured from one of the edges of the sensor to the opposite end. Biasing voltage refers to the voltage used to provoke a current of electrons that will be deflected via Lorentz Force (1). The offset represents the measured voltage when no magnetic field affect the sensor due to manufacturing imperfections. Ideally, the offset of the sensor should be $0 \mathrm{~V}$. However, manufacturing imperfections make this offset a finite number. Similarly, at a 
magnetic field of $1.5 \mathrm{mT}(15 \mathrm{G})$, the sensor responds with an appropriate Hall voltage. With these parameters in mind, a COMSOL simulation was developed with nominal sensor parameters.

Subsequent subsections (2.3.1-2.3.7) describe in detail how the simulation was setup, which includes Global Parameters, Geometry, Materials, Physics, Mesh, and Study type, followed by Results. Any bold font henceforth denotes a COMSOL function or term from the Model Builder window. An example of this window is show in Figure 2-7.

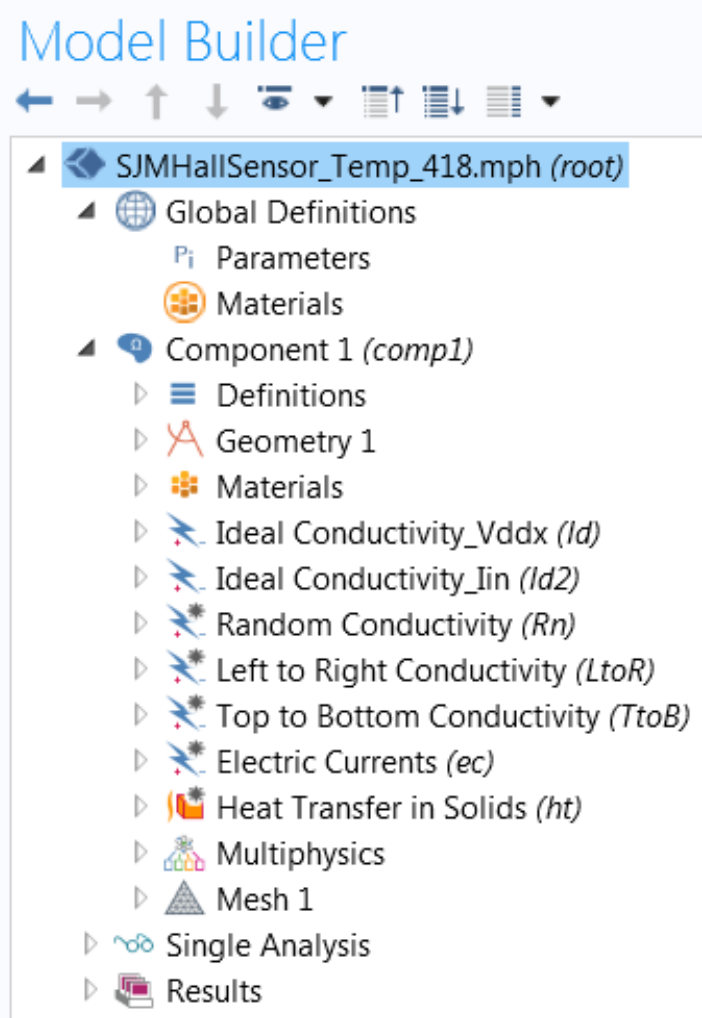

Figure 2-7. Example of COMSOL's Model Builder window. 


\subsubsection{Global Parameters}

Global Parameters are analogous to global variables; they can be manipulated or read by every entity in COMSOL. The material properties parameters from Table 2-1 were input to COMSOL in the form of Parameters from Global Definitions.

\subsubsection{Geometry}

Geometry gives the user diverse tools to model the shape of interest. The sensor Geometry was constructed by making a 100x56 $\mu \mathrm{m}$ Rectangle, with base centered at $(0,0)$ in the $x-y$ plane. Then, this shaped was copied and rotated by $90^{\circ}$ with the Rotate function, with the center of rotation at the origin $(0,0)$. The original shape was conserved. Finally, these two rectangles were joined together with COMSOL's Form Union function, which makes the two rectangles a single entity or geometry in COMSOL. The resulting geometry is shown below, in Figure 2-8.

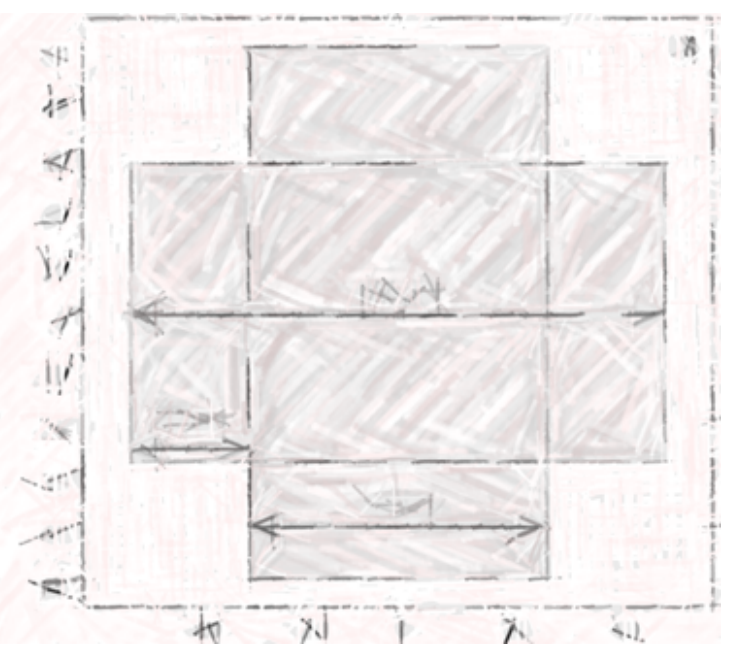

Figure 2-8. Resulting Hall device geometry used in COMSOL. Image has been blurred due to NDA. 


\subsubsection{Materials}

Materials requests the relevant material properties for the physics being simulated. COMSOL has an internal library of material properties. In this case, COMSOL's Silicon was selected, which imported relative permittivity of silicon of 11.7.

\subsubsection{Physics}

In Physics, the user can import and tune whichever type of physics might apply to the simulation in question. One of the features of COMSOL is the simplicity in coupling physics together, simplifying complex simulations. However, since this simulation involves a user-defined equation, physics coupling might not be as straight-forward. Nonetheless, only Electric Currents physics was imported. This physics was applied to the geometry presented in Figure 2-8. As mentioned in last simulation for the validation of the magnetoconductivity model (15), even though this was a 2D simulation, COMSOL's Electric Current physics extrudes the geometry to a desired Out-of-plane thickness to make calculations, such as resistance instead of sheet resistance, possible. The first physics to consider from COMSOL's Electric Current is Current Conservation.

Current Conservation will be applied to the whole geometry from Figure 2-8. This physics introduces the continuity equation for charge conservation [9], current density [10], and electric field as gradient [11], namely

$$
\nabla \cdot \boldsymbol{J}=Q_{j}
$$




$$
\begin{gathered}
J=\boldsymbol{\sigma} \boldsymbol{E}+\boldsymbol{J}_{\boldsymbol{e}} \\
\boldsymbol{J}=-\nabla \mathrm{V}
\end{gathered}
$$

The electrical conductivity was modified to anisotropic with the magnetoconductivity model (15). The constitutive relation was relative permittivity [12], with silicon's relative permittivity of 11.7 .

The next physics used was Electric Insulation, which states that there is no current density normal to the Hall sensor edges that are not considered terminals. In mathematical terms, Electric Insulation is presented as such

$$
\mathbf{n} \cdot \boldsymbol{J}=0
$$

Initial Value for voltage throughout the sensor was set to zero. However, this will be overridden by Terminals and Floating Potentials, as described next. The leftmost edge of the sensor was considered a voltage Terminal with the required biasing voltage. Similarly, the rightmost edge was considered Ground, which implies that the voltage at this point is zero. The top and bottommost terminals were considered two distinct Floating Potentials with zero current and, due to sensor symmetry, initial value for voltage of half of the biasing voltage. The relevant equation for this physics is shown below. All physics applied are summarized in Figure 2-9.

$$
\int_{\partial \Omega}-\mathbf{n} \cdot \boldsymbol{J} d S=0
$$




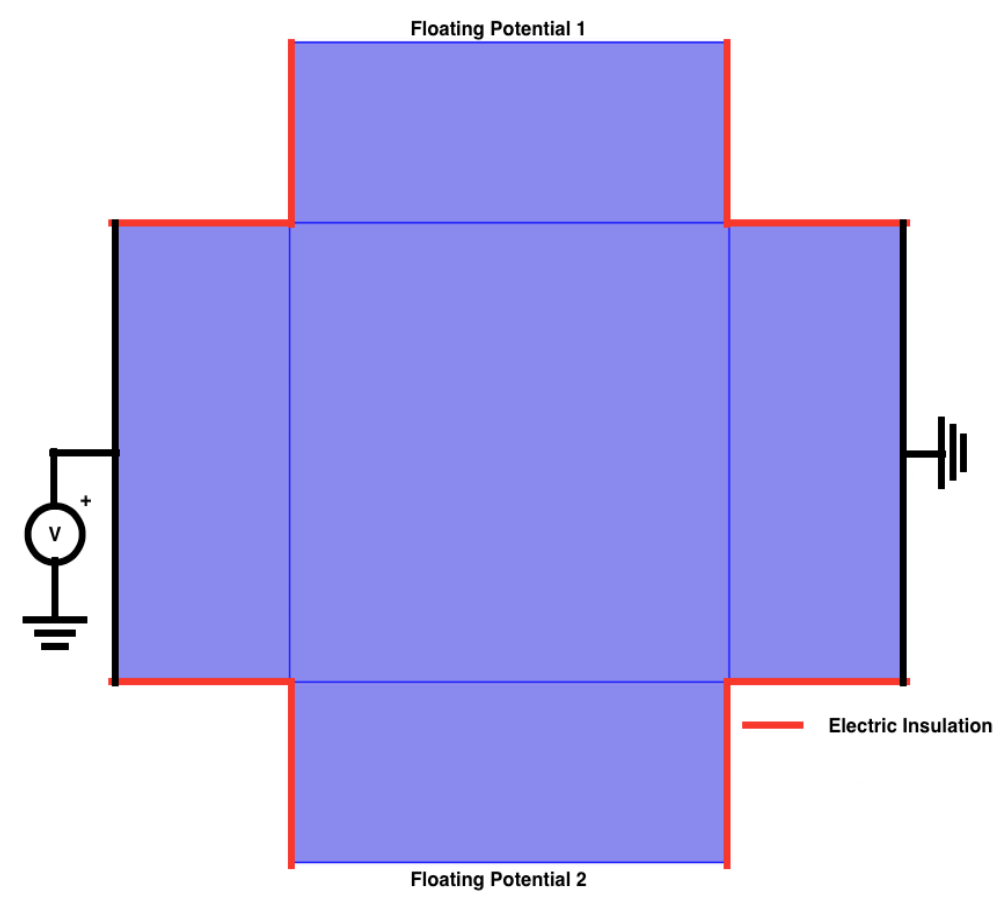

Figure 2-9. COMSOL Physics applied to Hall device geometry. The purple area represents where Current Conservation is present.

\subsubsection{Mesh}

Mesh defines the amount and size of domain and boundary elements. Even though the triangular mesh is the most commonly used type of mesh, different physics are optimized with different mesh types. As a rule of thumb, starting with a coarse mesh can save a lot of time, since the software has to compute less solutions. A Free Triangular Mesh was selected, with maximum element size of $1 \mu \mathrm{m}$, minimum element size of $0.002 \mu \mathrm{m}$, maximum element growth rate of 1.1 , curvature factor of 0.2 , and resolution of narrow regions of 1 . This yields an extremely fine mesh of 20448 domain elements and 624 boundary elements, which helps with simulation accuracy. The resulting Mesh is shown in Figure 2-10 (a) and (b). 


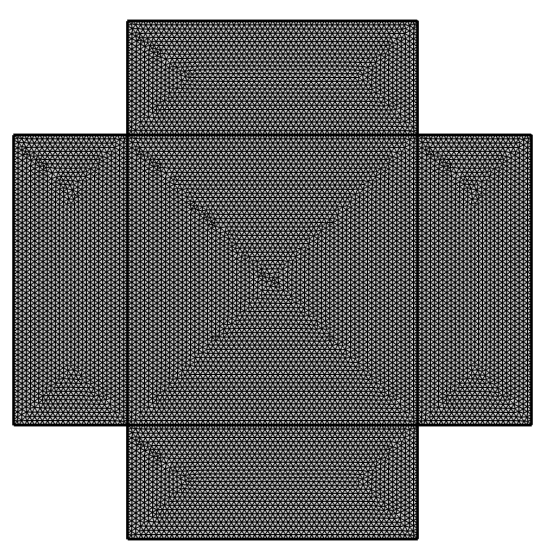

(a)

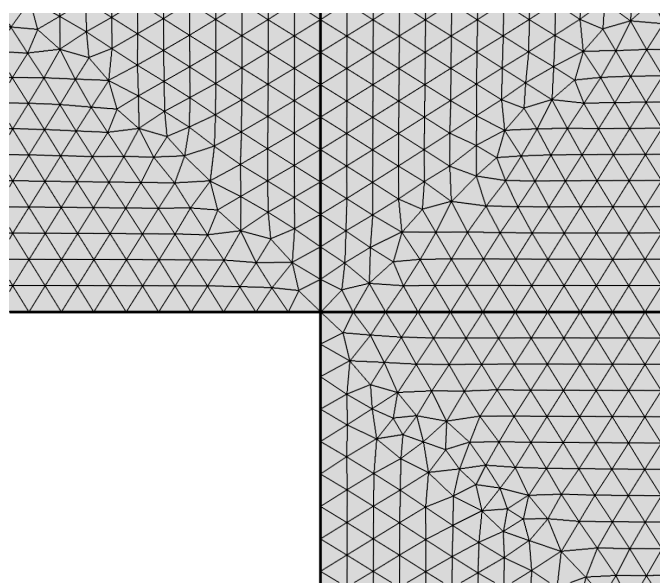

(b)

Figure 2-10. (a) Mesh built for entire geometry. (b) Close-up of bottom-left corner of mesh to show details. The mesh consists of 20448 domain elements and 624 boundary elements.

\subsubsection{Study}

Study gives the user freedom to select what type of analysis to perform on the model. For example, for the Electric Currents physics, the typical preset studies are Frequency Domain, Small-Signal Analysis, Stationary, and Time Dependent. Furthermore, Study dictates the order in which the solver computes solutions and allows to perform Parametric Sweeps. The goal was to reach $\Omega$ without magnetic field, $\quad \mu \mathrm{V}$ at $1.5 \mathrm{mT}$ and a sensor offset of $\mathrm{mV}$. To achieve a sensor resistance of $\Omega$, a COMSOL Parametric Sweep Stationary study was made to analyze the relationship between sensor resistance, without magnetic field, and electron mobility and/or density. Similarly, to achieve the desired Hall voltage of $\mu \mathrm{V}$ at $1.5 \mathrm{mT}$, an additional COMSOL study was performed to analyze the relationships between Hall voltage, at fixed magnetic field, and electron mobility and/or density. The electron mobility and density were swept with a 
Parametric Sweep while probing the resistance of the sensor and the Hall voltage at a few different magnetic fields.

\subsubsection{Results}

To obtain the desired sensor parameters, Global Evaluations for resistance and Hall voltage were required. For resistance, the Terminal voltage was divided by the Terminal current, a value calculated automatically by COMSOL. The difference between Floating Potentials 1 and 2 yield the Hall voltage, or offset in the absence of magnetic fields. Part of the tabulated results from these Global Evaluations yielded the relationship between sensor resistance and electron mobility and density (Figure 2-11), as well as the Hall voltage at a constant magnetic field with respect to changing electron mobility and density (Figure 2-12), and finally offset with respect to these two material properties (Figure 2-13).

Figure 2-11 shows an inverse relationship between sensor resistance and electron mobility; this stems from the conductivity equation (7). In the other hand, Figure 2-12 shows that the Hall voltage is independent of electron density, but highly dependent on electron mobility. According to these two graphs, choosing electron mobility of $0.04245 \mathrm{~m}^{2} /(\mathrm{V} \mathrm{s})$ and electron density of $1.956 \times 10^{23} \mathrm{~m}^{-3}$ yield the desired results for device resistance and Hall voltage response to $1.5 \mathrm{mT}$. Nonetheless, Figure 2-13 shows that sensor offset is not affected by changes in electron density nor mobility, and offset remains constant around $60 \mathrm{nV}$. Since the 
offset is measured from Floating Potentials (20), the small discrepancies in offset most likely arise from the mesh elements being misaligned.

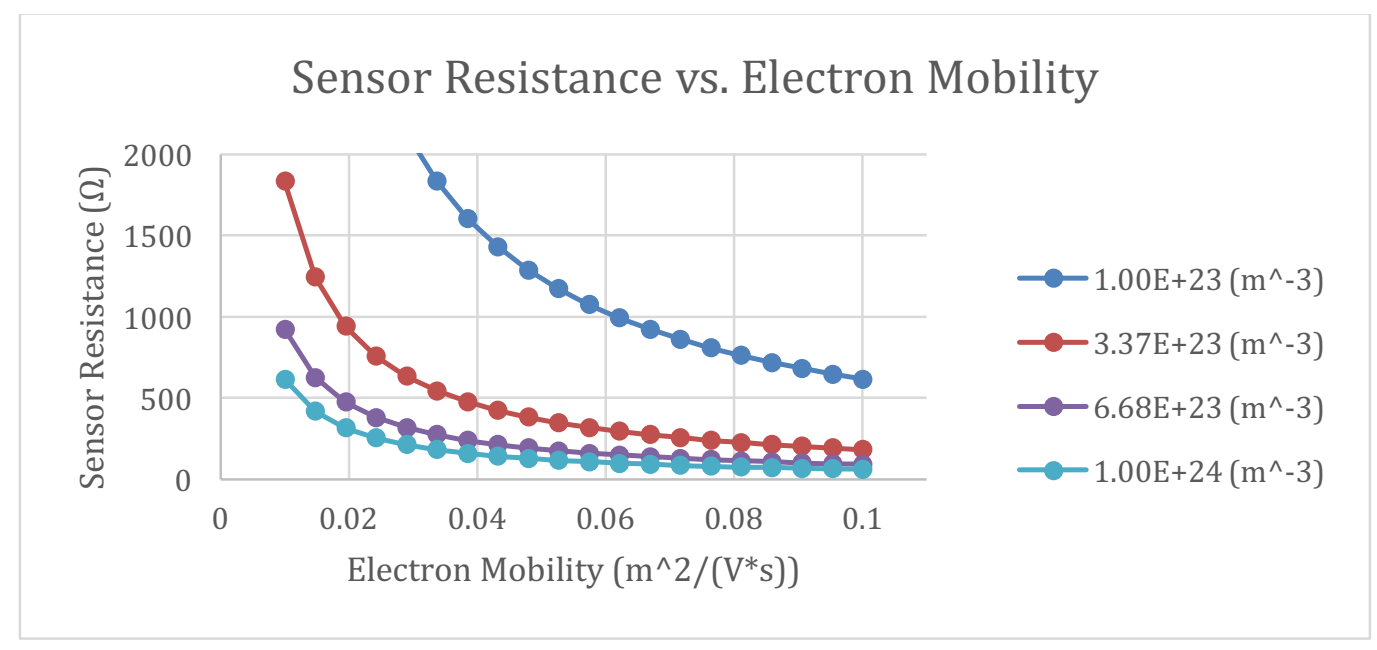

Figure 2-11. Sensor resistance vs. electron mobility with different electron density values.

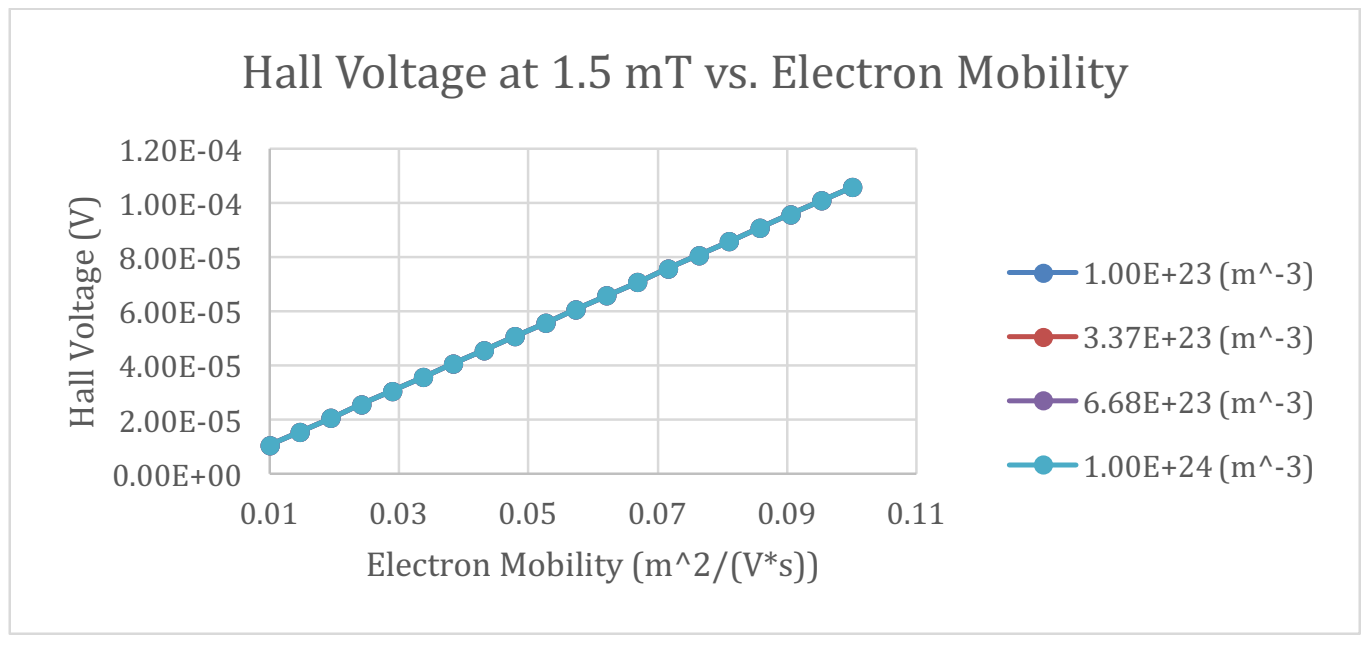

Figure 2-12. Sensor Hall voltage at constant $1.5 \mathrm{~T}$ magnetic field vs. electron mobility with different electron density values. Target value is reached with electron mobility value of $0.0432 \mathrm{~m}^{2} /(\mathrm{V} \mathrm{s})$. Note that all electron density lines overlap, hence proving that this magnetoconductivity model disregards electron density for Hall voltage calculation. 


\section{Sensor Offset vs. Electron Mobility}

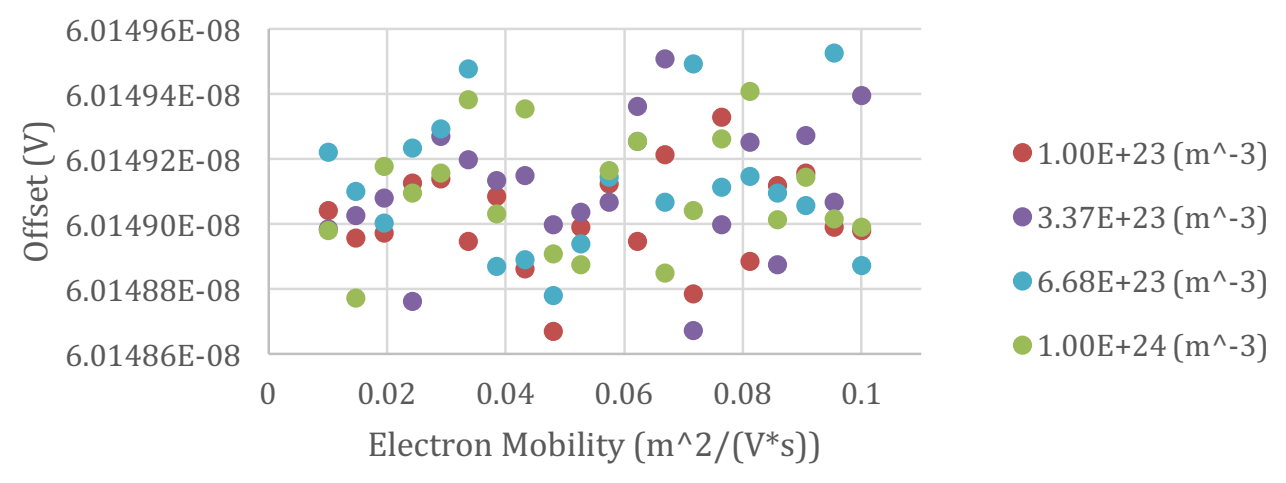

Figure 2-13. Sensor offset with respect to varying electron density and mobility values. The Sensor Offset axis is not a mistake. The extremely small variation in offset is most likely due to symmetrical mesh element misalignment.

Two out of the three targets were reached. Namely, a Hall sensor resistance of $\Omega$ and Hall voltage response to $1.5 \mathrm{mT}$ of $\mu \mathrm{V}$ was reached. The electron mobility and density values match the ranges as specified by SJM. However, sensor offset has not yet been achieved. Offset stems from sensor and hence fabrication imperfections. To attain a better understanding of the offset, fabrication imperfections must be somehow accounted for in the COMSOL model.

\subsection{Gaussian Junction Depth}

Even though transistor fabrication has increased in reliability over the past few decades, imperfections in transistors are still present. One of these imperfections arises when doping silicon via ion implantation. 
During ion implantation, ions penetrate semiconductor crystals to depths of thousands of angstroms (hundreds of nanometers). Thousands of interactions can occur in this process. Furthermore, the incident and target ions have masses of the same order of magnitude. It is possible for the incident ion to be scattered at a large angle relative to its incident velocity. As a result, nuclear interactions cannot be treated as a continuum. Instead, they must be treated as a series of discrete events. The angle at which the ion is scattered will depend on the impact parameter on the masses and relative positions of the two ions. This means that the result of any interaction depends on all of the interactions that occurred previously, back to the first atomic layer of the solid. Since the ions are uniformly distributed over the surface of the wafer as they enter, a statistical distribution of depths will result. Gaussian distributions can be used to model the range of depths that an ion might reach. Thus, the impurity concentration as a function of depth in an amorphous solid will be given by

$$
N(x)=\frac{\phi}{\sqrt{2 \pi} \Delta R_{p}} e^{-\frac{\left(x-R_{p}\right)^{2}}{2 \Delta R_{p}^{2}}}
$$

where $R_{p}$ is the projected range, $\Delta R_{p}$ is the standard deviation of the projected range, and $\phi$ is the dose $[13$, Ch. 6$]$.

It follows, then, to enhance the model of the Hall effect sensor due to the depth of ion implantation. The Gaussian distribution model for the depth of ion implantation 
might translate into a Gaussian distribution of the device conductivity. This means that the conductivity of the sensor can be modeled as Gaussian noise distributions areas within the sensor, and hence accounting for junction depth imperfections within the Hall sensor model. This model addition should lead to a more realistic voltage offset as opposed to the results obtained previously in the ideal model.

\subsubsection{Simulation Setup}

Steps outlined in sections 2.3.1-2.3.7 still apply for this new simulation, however some minor changes, explained next, were required to implement this Gaussian ion implantation behavior into COMSOL. The goal was to achieve a sensor offset of approximately $\mathrm{mV}$, and perform a Monte Carlo analysis on sensor offset population behavior. To do so, the sensor geometry discretized by dividing it into $1 \mu \mathrm{m}^{2}$ squares and each of these squares exhibited slightly different conductivity behavior based on a random value obtained from COMSOL's Random function.

The built-in Random function was set to follow a normal (Gaussian) type of distribution. Furthermore, the function was centered around a mean of 1 , since the values resulting from Random represent a certain percentage variation of the sensor's innate conductivity $\sigma_{0}$ in each of the squares that the geometry was divided. A Global Parameter for the standard deviation of this distribution was initialized. Random uses a user-defined number of arguments. In this case, three different arguments were required, as explained next. The output of the Random function is seen in Figure 2-14. 

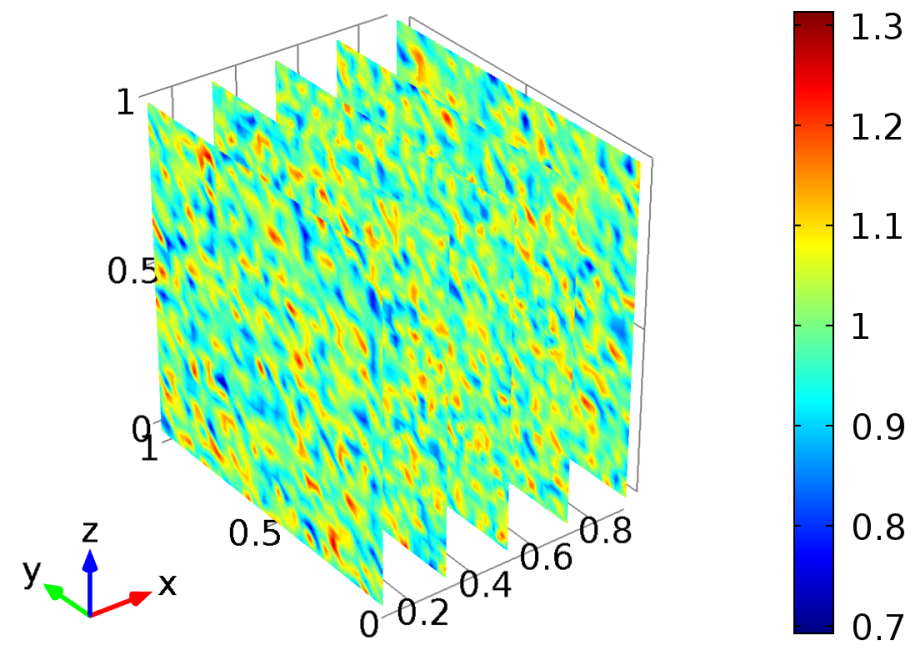

Figure 2-14. Output of Random function. Notice that the random distribution is centered around 1. The different planes or slices shown represent the distinct spatial variations introduced via a random seed, which represents the $3^{\text {rd }}$ argument for this function.

First, under Component $>$ Definitions, a Variable was initialized for the area size that would represent a different conductivity based on the Gaussian distribution. Additional variables for discrete spatial variation in the $x$ - and $y$-directions were required, using the ceiling function, to emulate distinct ion implantation imperfections in each of the squares that the sensor was divided into, as shown below

$$
X, Y(x, y)=\operatorname{ceil}\left(\frac{x, y}{\text { GridSize }}\right)
$$

where $X$ and $Y$ are discrete values of $x$ and $y$, respectively, and GridSize represents the size of the side of the square used for the discretization of the Hall sensor geometry $\left(1 \mu \mathrm{m}^{2}\right)$. To attempt to replicate sensor population behavior, a 
random seed Global Parameter was introduced, which allowed different sensor spatial variations for Monte Carlo simulations. The spatial variations and random seed represent three distinct arguments for Random. The resulting conductivity distribution can be seen in Figure 2-15. Finally, a Parametric Sweep was performed on the standard deviation of the Gaussian conductivity from 1 to $20 \%$ and on 250 different random seed values. Results for this simulation are shown in next section.

\subsubsection{Simulation Results and Discussion}

The sensor offset and the sensor's population behavior was reached with the results from this simulation according e-mail conversations and meeting notes with SJM Analog IC Design Engineer and Technical Advisor for this project, Frank Wei [14]. Figure 2-16 and Table 2-2 show a histogram distribution of the Monte Carlo analysis results on the sensor offset. The data's mean is $249 \mu \mathrm{V}$, with a standard deviation of $1.78 \mathrm{mV}$. 


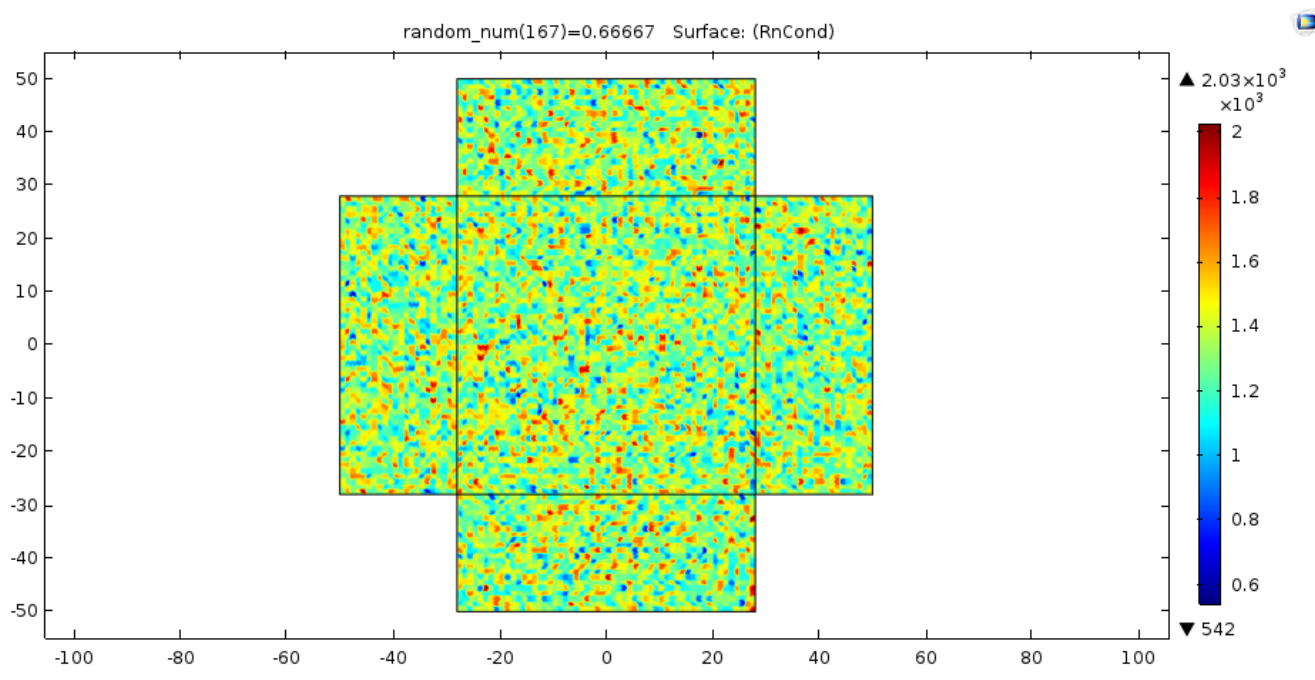

Figure 2-15. Example of conductivity model affected by discretized ion implantation imperfections. random_num(167) $=0.66667$ denotes the random seed used. A different seed produces a different conductivity distribution from the one seen above. The color bar on the right represents the conductivity value in $\mathrm{S} / \mathrm{m}$.

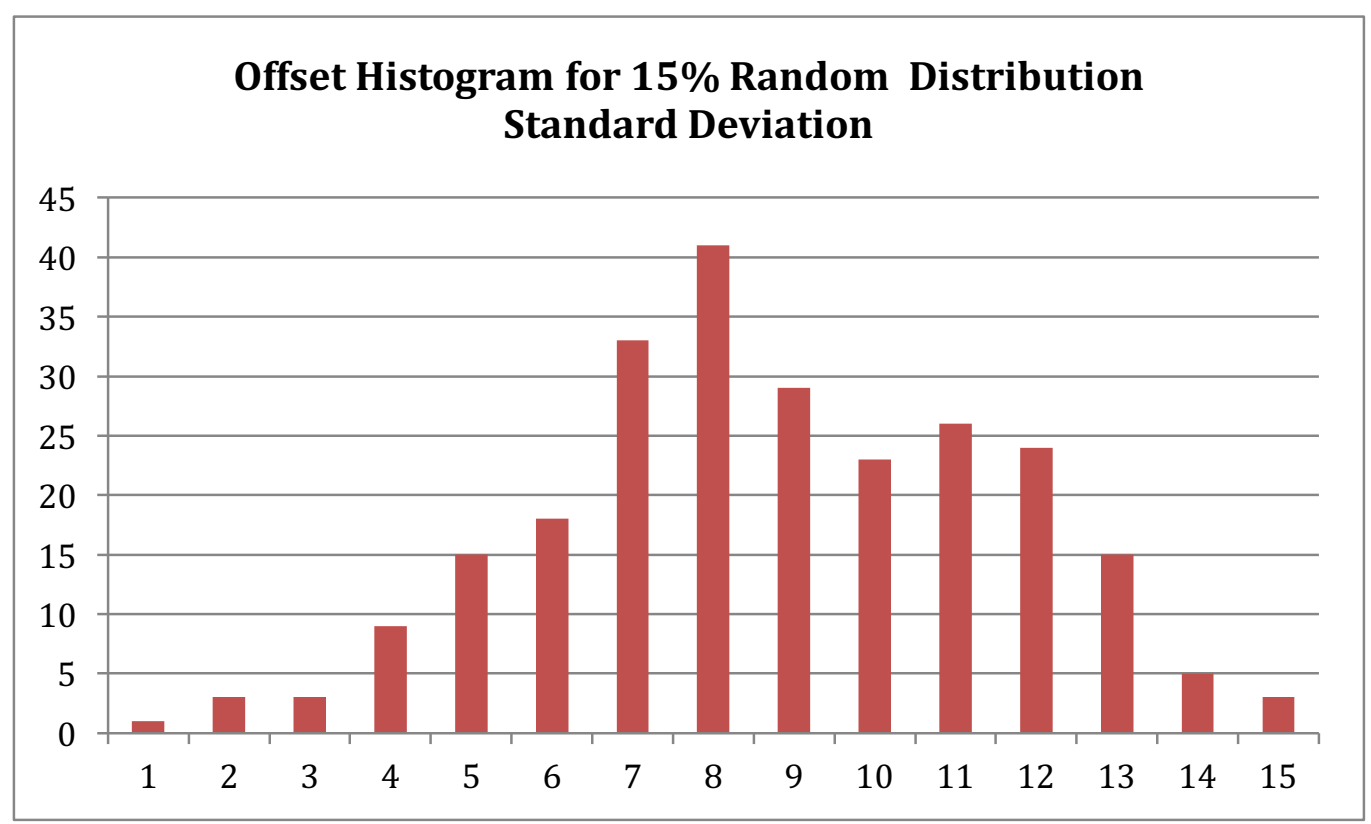

Figure 2-16. Histogram distribution for sensor offset values. The numbers in the $x$-axis denote bin numbers as per Table 2-2. 
Table 2-2. Histogram bin frequency distribution with respective offset values.

\begin{tabular}{|c|c|c|}
\hline \multicolumn{3}{|c|}{ Offset } \\
\hline Bin \# & Bins (V) & Frequency \\
\hline 1 & -0.00432 & 1 \\
\hline 2 & -0.0037 & 3 \\
\hline 3 & -0.00307 & 3 \\
\hline 4 & -0.00244 & 9 \\
\hline 5 & -0.00182 & 15 \\
\hline 6 & -0.00119 & 18 \\
\hline 7 & -0.00056 & 33 \\
\hline 8 & 6.47E-05 & 41 \\
\hline 9 & 0.000692 & 29 \\
\hline 10 & 0.001318 & 23 \\
\hline 11 & 0.001945 & 26 \\
\hline 12 & 0.002572 & 24 \\
\hline 13 & 0.003199 & 15 \\
\hline 14 & 0.003826 & 5 \\
\hline 15 & 0.004453 & 3 \\
\hline & More & 2 \\
\hline
\end{tabular}

\subsection{Ramping Gaussian Junction Depth}

Considering that ion implantation usually happens at an angle to prevent ion channeling deeper into the wafer [15], [16, Fig. 2] it was hypothesized that the implantation might be stronger in one end of the sensor and weaker at the other end. For this reason, it was decided to implement a ramp function to the conductivity; meaning that the conductivity of one end of the sensor should steadily increase/decrease as the other end of the sensor is approached, while still presenting the Gaussian distribution in conductivity to match similar results presented in Figure 2-17. Two cases of tilting were explored; the top-to-bottom 
(TtoB) and the left-to-right (LtoR) ramps. With these conditions, the offset voltage of the Hall sensor should be greater for the top-to-bottom ramping conductivity.

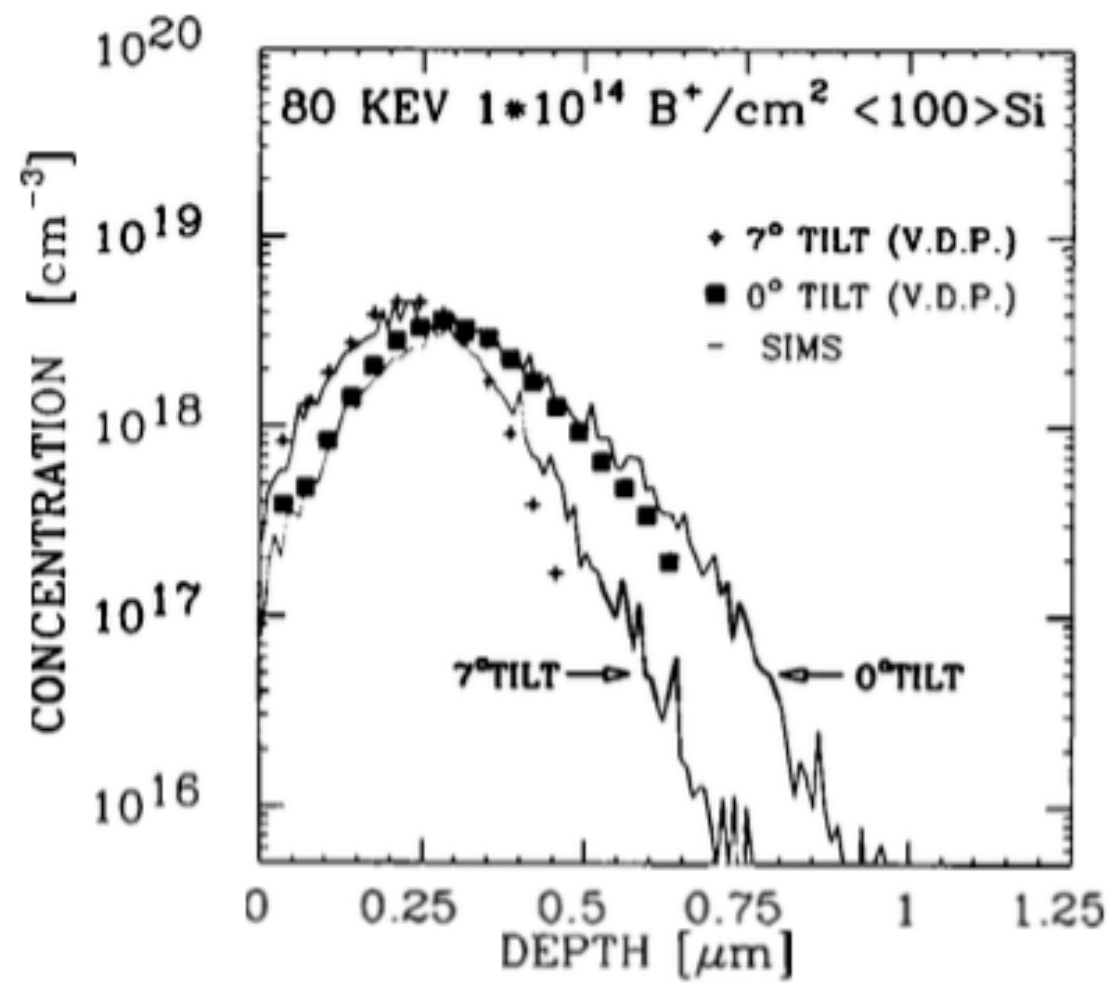

Figure 2. Carriers and chemical concentration profiles for $7^{\circ}$ and $0^{\circ}$ boron implants into a $\langle 100\rangle$ silicon target at a fluence of $1 \times 10^{14} \mathrm{~cm}^{-2}$.

Figure 2-17. Carrier concentration profile [16, Fig. 2] showing the advantage of using a $7^{\circ}$ tilt in comparison to no tilt.

\subsubsection{Simulation Setup}

Two custom Analytic functions were added to COMSOL's Component Definitions. The Analytic function provides a user-defined expression while still controlling arguments and plot parameters. In this case, the following expression was utilized 


$$
\mathrm{X}, \mathrm{YRamp}(\mathrm{x}, \mathrm{y})=\frac{2(x, y)}{l} \sigma+1
$$

where $x$ and $y$ are spatial variables in the $x$-and $y$-axis, respectively; I denotes the sensor length, and $\sigma$ represents ramp slope. The plot parameters for this function, i.e. restrictions for the plot arguments, have a limits of $\pm 1 / 2$. This means that the ramp function will only work within the sensor geometrical parameters. XRamp $(x)$ yields the LtoR ramp, while YRamp(y) yields the TtoB one. Examples of these ramping conductivities can be found in Figure 2-18 (a) and (b).

In this case, the ramp and Gaussian standard deviations were both swept at $1 \%$ and $10 \%$ via a Parametric Sweep. Since this model is supposedly an addition to the Gaussian model, a Monte Carlo analysis is required to observe offset population behavior. Results from using $T$ toB and LtoR ramping conductivities are shown in the next section.

\subsubsection{Simulation Results and Discussion}

Figure 2-18 (a) and (b) show the TtoB and LtoR ramping conductivity results on the sensor. Table 2-3 summarizes statistics from the population of simulations for TtoB and LtoR ramping functions, while Table 2-4 presents the average offsets and percent difference caused by changes in the Gaussian or ramp percent deviations. In both ramp functions, the average offset is mostly dependent on Gaussian noise standard deviation. Surprisingly, if both variables are set to deviate by $10 \%$, the total percent change is less than the case of only modifying the Gaussian noise. For both ramp cases, the Gaussian ramp introduces a maximum 
of tens of microvolts. For this matter, the Gaussian ramp functions were not investigated further.

Table 2-3. Offset statistical summary of TtoB and LtoR ramping functions with respect to Gaussian random noise standard deviation and ramp slope.

\begin{tabular}{clll}
\hline $\begin{array}{c}\text { Gaussian-Ramp } \\
\text { (Std. Deviation \%-Ramp Slope \%) }\end{array}$ & Offset (V) & TtoB Offset (V) & LtoR Offset $(\mathrm{V})$ \\
\hline \multirow{2}{*}{$0.01-0.01$} & Average & $1.54942 \mathrm{E}-06$ & $1.53262 \mathrm{E}-06$ \\
& Std.Dev. & $2.99958 \mathrm{E}-05$ & $3.00125 \mathrm{E}-05$ \\
\hline \multirow{2}{*}{$0.01-0.1$} & Average & $1.59287 \mathrm{E}-06$ & $1.41822 \mathrm{E}-06$ \\
& Std.Dev. & $3.00612 \mathrm{E}-05$ & $3.01474 \mathrm{E}-05$ \\
\hline \multirow{2}{*}{$0.1-0.01$} & Average & $1.47643 \mathrm{E}-05$ & $1.50807 \mathrm{E}-05$ \\
& Std.Dev. & 0.000341757 & 0.000341766 \\
\hline \multirow{2}{*}{$0.1-0.1$} & Average & $1.15355 \mathrm{E}-05$ & $1.44801 \mathrm{E}-05$ \\
& Std.Dev. & 0.000342596 & 0.000341572 \\
\hline
\end{tabular}

Table 2-4. Effects on sensor offset from changes in standard deviation and ramp from $1 \%$ to $10 \%$.

\begin{tabular}{ccccc}
\hline Change in: & TtoB $\Delta$ Offset $(\mathrm{V})$ & $\begin{array}{c}\text { TtoB } \% \text { Diff. } \\
\text { vs. } 1 \%\end{array}$ & LtoR $\Delta$ Offset $(\mathrm{V})$ & $\begin{array}{c}\text { LtoR } \% \text { Diff. } \\
\text { vs. } 1 \%\end{array}$ \\
\hline Ramp & $4.35 \mathrm{E}-08$ & $3 \%$ & $1.14 \mathrm{E}-07$ & $7 \%$ \\
Gaussian & $1.32 \mathrm{E}-05$ & $853 \%$ & $1.35 \mathrm{E}-05$ & $884 \%$ \\
Both & $9.99 \mathrm{E}-06$ & $645 \%$ & $1.29 \mathrm{E}-05$ & $845 \%$
\end{tabular}


random num $=1$, StdDev $=0.1$, StdDevR $=0.1$ Surface: Electrical conductivity, xx component (S/m)

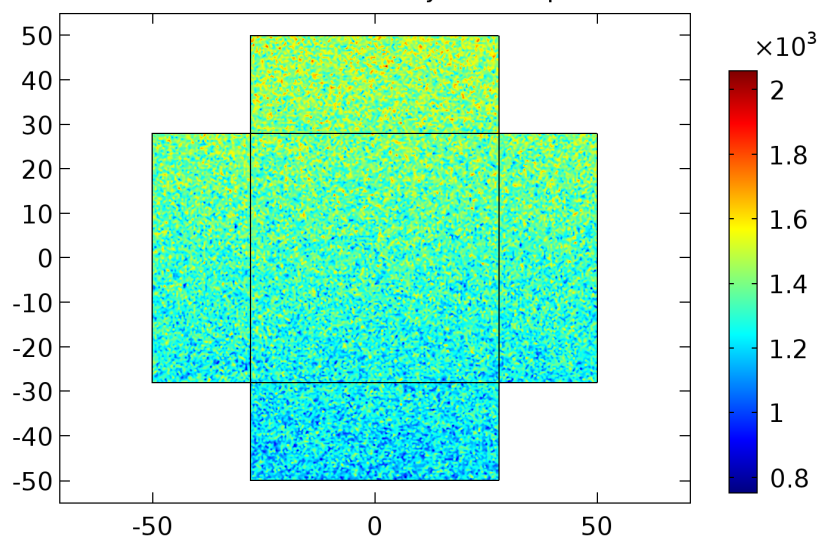

(a)

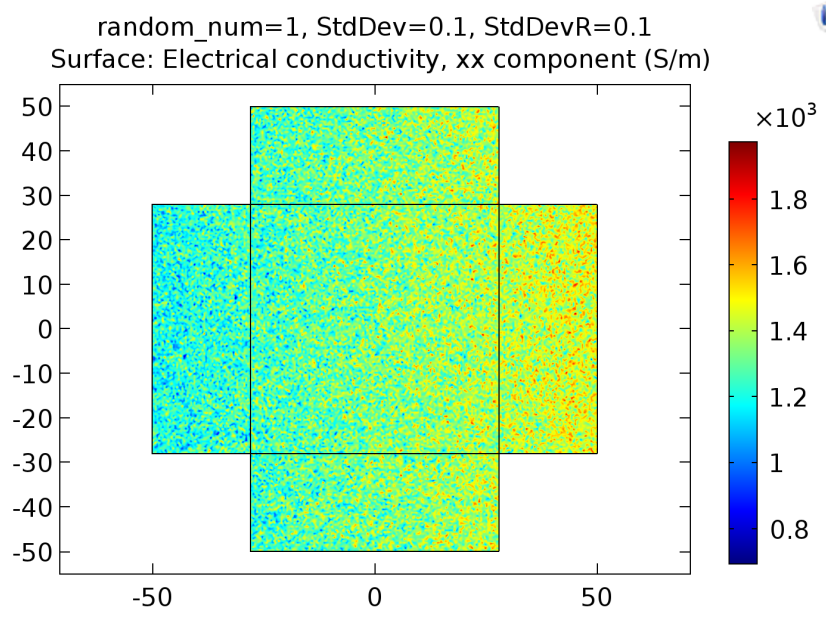

(b)

Figure 2-18. (a) TtoB ramping conductivity example. (b) LtoR ramping conductivity example. random_num indicates the seed used for the Gaussian noise. The Gaussian has a standard deviation of $10 \%$. The ramp function has a slop of $10 \%$ of the original conductivity.

\subsection{Hall Effect Temperature Dependence Progress}

Remembering that the Hall sensor is within the pacemaker which will be inside a human body, operation of the sensor based on temperature should also be 
considered. Normally, the core temperature of a human body ranges from $28^{\circ} \mathrm{C}$ to $44^{\circ} \mathrm{C}$, including extreme cases of hypo- and hyperthermia [17]. Hence, linearized conductivity should be applied to this model. As noted in the isotropic conductivity equation (8), a transversely isotropic material is characterized by a matrix whose diagonal terms are equal.

Resistance and temperature are directly proportional to each other in various conducting devices. This mathematical model is often referred to the linearized conductivity or linearized resistivity model and it is described by

$$
\sigma(T)=\frac{1}{\rho(T)}=\frac{\left(\mu_{e} n_{e}+\mu_{h} n_{h}\right) q}{1+\alpha\left(T-T_{r e f}\right)}
$$

where $\alpha$ is the temperature coefficient, typically in units of $\mathrm{ppm} /{ }^{\circ} \mathrm{C}, \mathrm{T}_{\text {ref }}$ refers to a reference temperature used when making conductivity measurements and it usually is room temperature $\left(24^{\circ} \mathrm{C}\right)$. Considering that electron mobility $\left(\mu_{\mathrm{e}}\right)$ and electron density $\left(n_{e}\right)$ are typically much greater than hole mobility and density $\left(\mu_{h}\right.$ and $n_{h}$, respectively), the latter terms can be ignored. Further, the magnetoconductivity model that gives rise to the Hall effect must be modified to include temperature dependence. One intuitive way to include this behavior to the model is by appending the temperature relationship to the magnetoconductivity model:

$$
\boldsymbol{\sigma}\left(H_{z}, T\right)=\frac{\mu_{e} n_{e} q}{\left(1+\left(\mu_{e} H_{z}\right)^{2}\right)\left(1+\alpha\left(T-T_{r e f}\right)\right)}\left[\begin{array}{ccc}
1 & -\mu_{e} H_{z} & 0 \\
\mu_{e} H_{z} & 1 & 0 \\
0 & 0 & 1
\end{array}\right]
$$

One can further imply that the linearized conductivity model leads to a more common relationship between temperature and resistance 


$$
R(T)=R_{0}\left(1+\alpha\left(T-T_{r e f}\right)\right)
$$

Hence, as temperature rises, the magnetoconductivity of the device decreases and resistance increases. Preliminary simulations show that Hall voltage seems to increase as well. However, according to theoretical calculations, B. Van Zeghbroeck mentions that the mobility of carriers is affected by temperature due to absorption or emission of acoustical phonons, with a resulting mobility proportional to $\mathrm{T}^{-3 / 2}$ in silicon [18], [19]. A phonon is a definite discrete unit or quantum of vibrational mechanical energy, just as a photon is a quantum of electromagnetic or light energy [20]. The Hall voltage equation, exchanging current via Ohm's law, results in

$$
V_{H}(T)=-\frac{V B}{R(T) n e d}
$$

Which shows that $V_{H} \propto T^{-3 / 2}$. Hence the simulation model must be modified accordingly to show a decrease in Hall voltage, but an increase in resistance as temperature rises.

\subsubsection{Simulation Setup and Results}

Noting that the FEM model's Hall voltage is governed by the term $\beta /\left(1+\beta^{\wedge} 2\right)$, where $\beta$ represents $\mu_{\mathrm{e}} \mathrm{H}_{\mathrm{z}}$, several temperature-dependent magnetoconductivity model variations were attempted through COMSOL and are summarized in the next graphs in Figure 2-19 and Figure 2-20. 


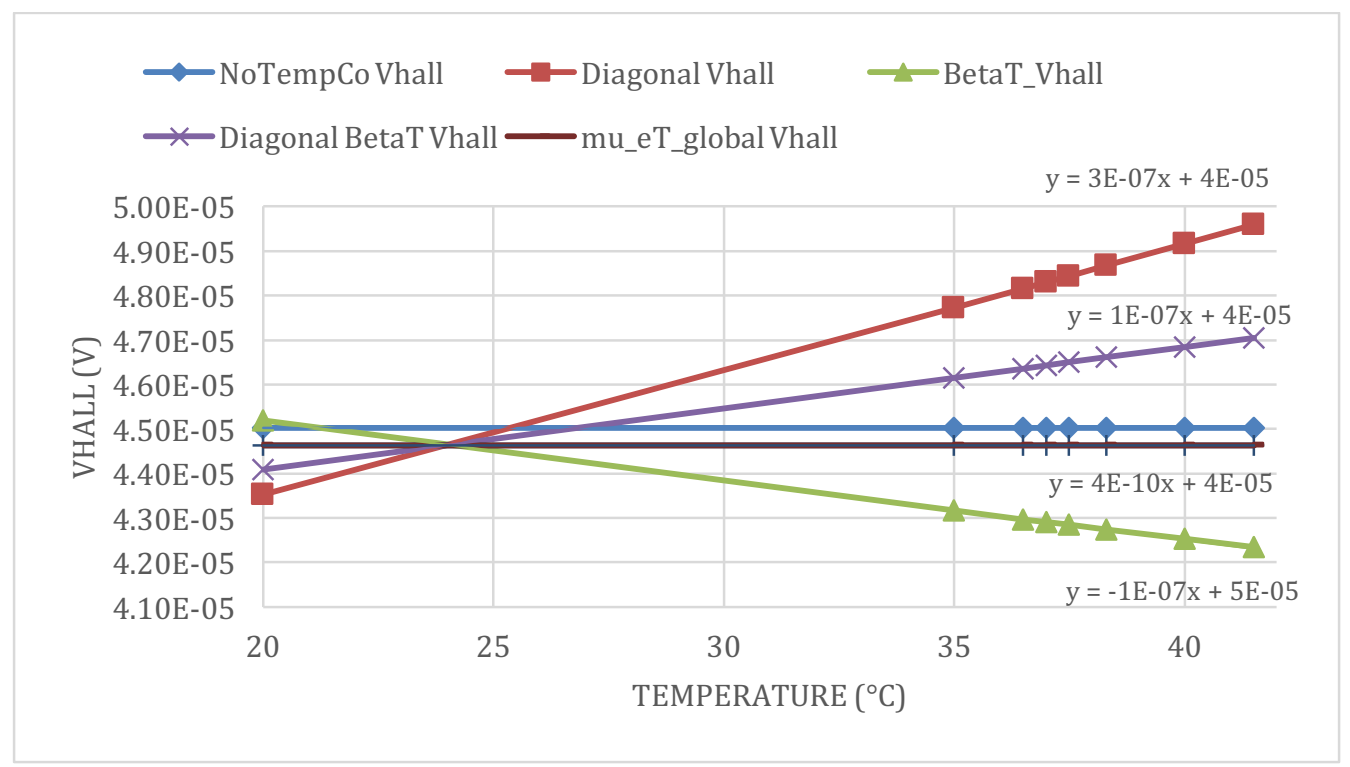

Figure 2-19. Summary of Hall voltage vs. temperature simulations. Equations displayed represent trend lines calculated by Excel. BetaT_Vhall was the only simulation that approached our expected results.

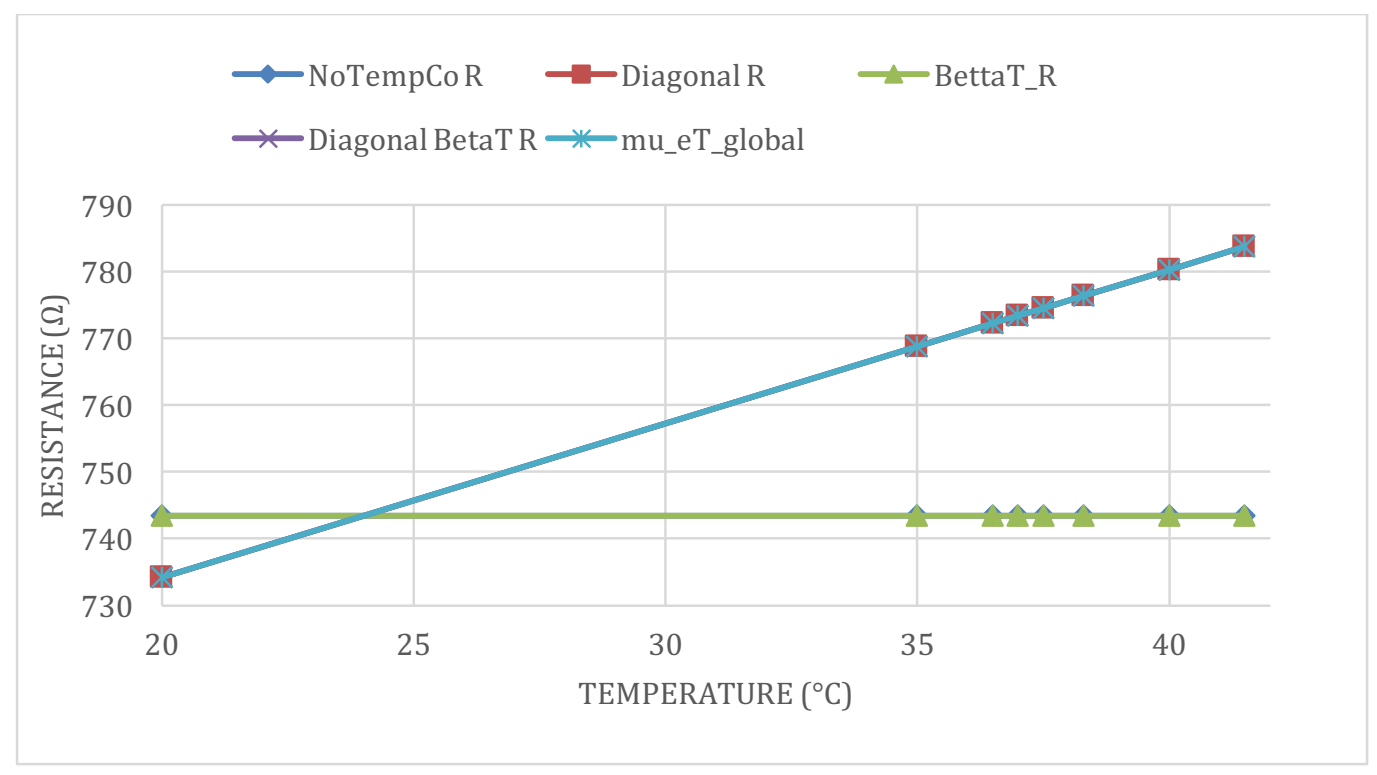

Figure 2-20. Summary of results for sensor resistance vs. temperature. Most magnetoconductivity models follow the increasing trend of mu_eT_global, which exception of NoTempCo and BettaT. 
Table 2-5. Magnetoconductivity models used for COMSOL simulations.

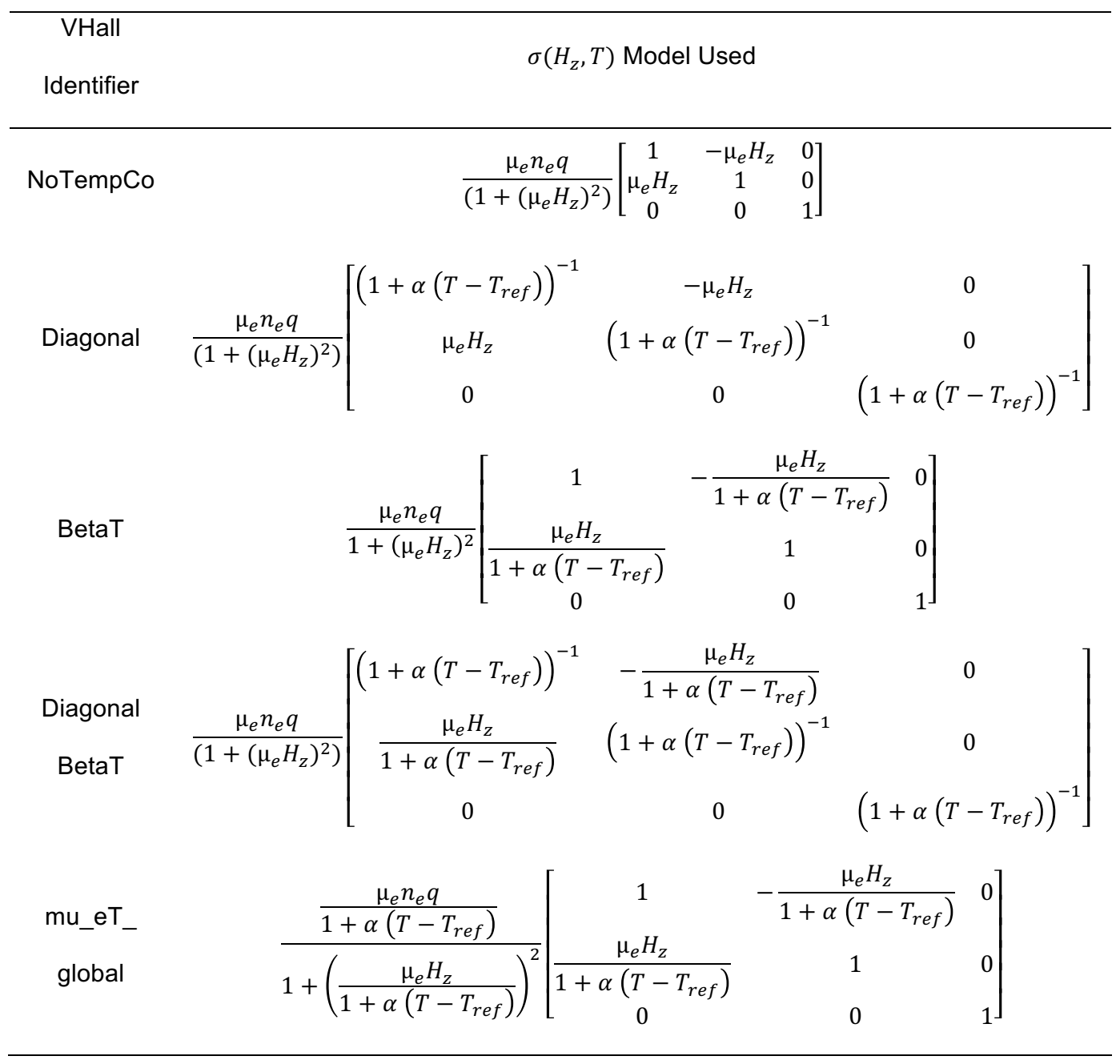

In Figure 2-19, the only model that significantly decreased with an increase temperature was the BetaT global model. Nonetheless, the resistance of this model seems to remain constant with an increase in temperature, as seen in Figure 2-20. This contradicts the initial hypothesis that resistance should increase with raising temperature. Hence, further modifications to the FEM magnetoconductivity model are required to match $\mathrm{V}_{\mathrm{H}} \propto \mathrm{T}^{-3 / 2}$. 


\subsection{Future Work}

Even though a 2D FEA model was favored over a $3 \mathrm{D}$ model due to lack of computing resources, in the end a $3 \mathrm{D}$ model will more accurately describe the overall behavior of the Hall sensor. This enhancement can be realized by modeling the Hall sensor inside the field of a multi-turn coil similar to the one used in the Helmholtz pair simulation (Figure 3-7).

More research should facilitate reaching the goal for sensor temperature dependency. A possible way to add temperature dependency to the model might be to enhance from 2D to 3D. Additionally, COMSOL has a Heat Transfer module, which can be coupled with the AC/DC module used in this project. The Heat Transfer module was not used in the 2D simulations since the conductivity of the device was customized, and hence affecting the physics coupling in unpredictable ways. Lastly, COMSOL has a Semiconductors module that could help once the model starts needing more intricate designs and complex atomic interactions.

Even though the current FEA Hall effect sensor simulation model applies only to SJM's sensor, the same magnetoconductivity model can be utilized to model alternative geometries. Contemporary literature on Hall effect devices seems to favor cross-shaped sensors [7], [39]. However, with more complex sensors and devices being researched and fabricated each day, simulations, such as the ones developed in this thesis, can be powerful tools for IC designers since it allows tuning of material properties and geometry for the theoretical device under 
investigation. This model provides an initial step on understanding the behavior of Hall effect devices. It also makes testing the effectiveness, and hence feasibility, of new Hall sensor geometries without having to physically build many prototype revisions. Every prototype iteration avoided with a simulation translates into saving of resources. 


\section{Conductive Ink Sensor}

In an attempt to research new technology while advancing the understanding of the Hall effect sensor, the printed electronics realm was explored. Thanks to the great help from Dr. Malcolm Keif and Bryce Beatty, from the Graphic Communication department at Cal Poly, different techniques for printing Hall sensors and their Hall effect sensitivity were explored.

\subsection{Introduction to Printed Electronics}

Printed electronics is an exciting new area for electronics manufacturing. Conductive and semiconductive materials, dielectrics, and other materials allow the manufacture of passive components, and sensors, among others. A printed electronic component typically consists of several layers of functional materials printed on top of each other.

Costs associated with mass-manufacturing printed electronics is considerably smaller in comparison to conventional electronics manufacturing. In the printing industry, the cost structure is different from traditional electronics technology, where labor and manufacturing techniques are the major costs in the end products due to the requirements of dedicated materials, doping compounds, encapsulation and so on for each type of devices. An extra advantage of PE is lower capital investment cost. It is estimated that a PE plant will cost $\$ 30$ million in comparison with $\$ 3$ billion for a silicon fabrication plant [21]. 
Nonetheless, low manufacturing costs comes at the price of performance. For a graphical comparison between, printed and conventional electronics, see Figure 3-1. Printed electronics have long switching losses and low integration density. However, given current consumer trends in the USA, with new versions of devices every year, printed electronics might someday become a more cost-effective manufacturing technique for this market. The printed electronics market is already valued at approximately 1 billion dollars. By 2016, until when the pilot phase is expected to last, market growth is likely to reach a volume of 5 billion dollars [22].

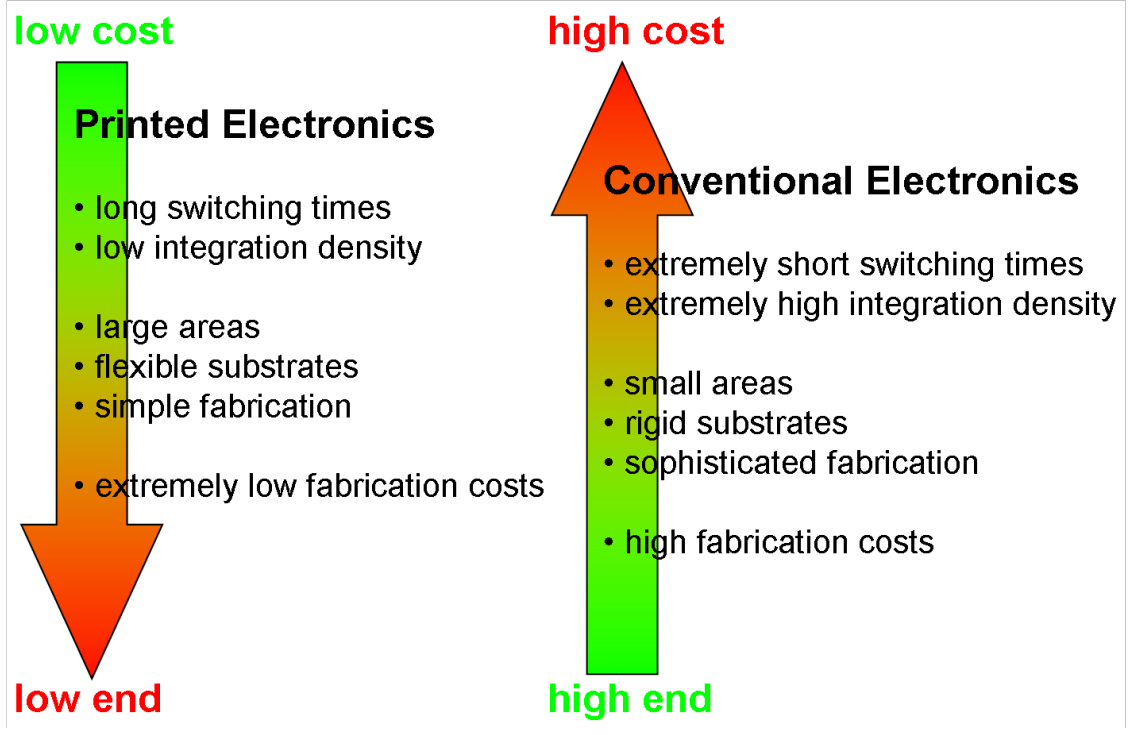

Figure 3-1. Comparison between printed and conventional electronics technologies [23]. 


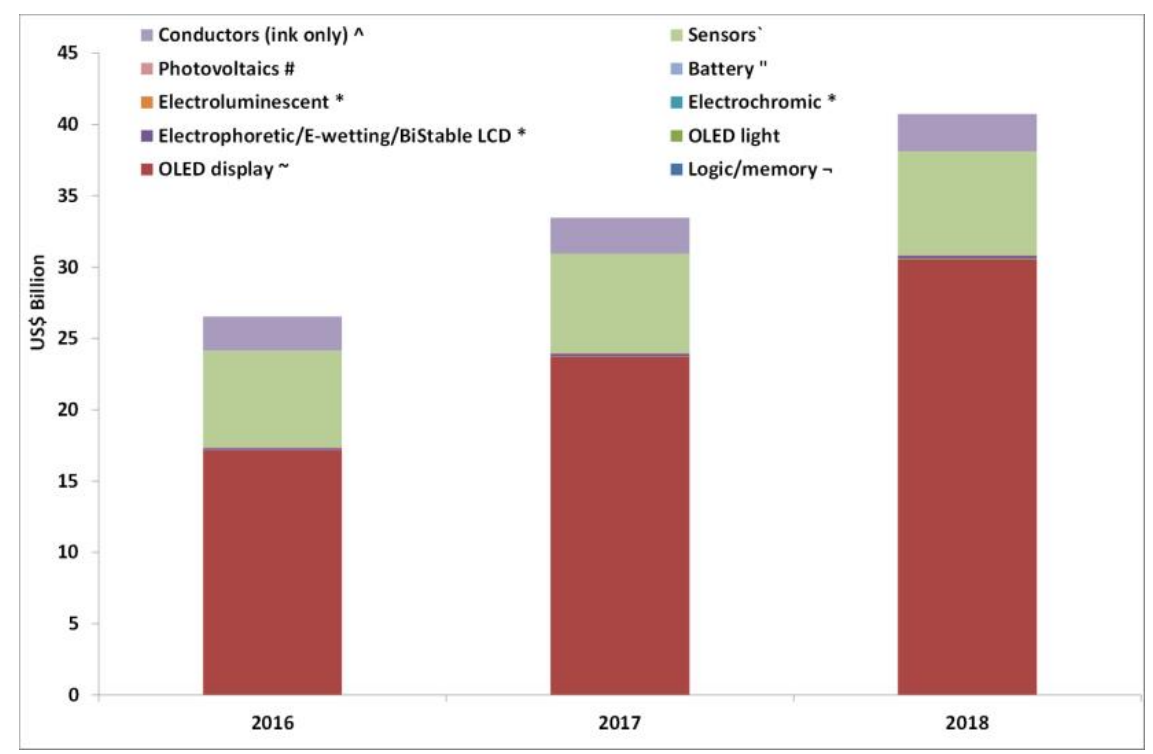

Figure 3-2. Printed electronics expected market cost (2016-2018) [24].

\subsection{Initial Printed Hall Sensor Considerations}

Recalling that the Hall voltage equation (5) is heavily dependent on the device thickness, the sensor thickness must remain as thin as possible while still allowing an even path for the flow of electrons. Although silver ink is typically used for printed electronics, its high charge carrier density of $1.070 \pm 0.001 \times 10^{28} \mathrm{~m}^{-3}[25]$ might make an impractical printed Hall sensor. An ink with a low charge carrier density should be used instead.

An alternative suggested by Dr. Keif was a heat curable carbon ink, with a sheet resistance of $120 \Omega /$ sq., at $25 \mu \mathrm{m}$ of thickness, yielding a resistivity of $3 \times 10^{-3} \Omega$ $m$ [26]. Due to unknown material properties such as charge carrier density and mobility, a point of reference is required for these two properties. Since the ink is 
carbon based, it was hypothesized that the ink should exhibit similar properties as graphite, which exhibits the following properties:

Table 3-1. Approximate graphite material properties [27], [28], [29, p. 61]. ${ }^{\dagger}$ Sheet resistance was obtained from manufacturer's datasheet [26].

\begin{tabular}{ccc}
\hline Property & Value & Unit \\
\hline Electron mobility & $20 \times 10^{3}$ & $\frac{\mathrm{cm}^{2}}{V \mathrm{~s}}$ \\
Hole mobility & $15 \times 10^{3}$ & $\frac{\mathrm{cm}^{2}}{\mathrm{Vs}}$ \\
Electron concentration & $5 \times 10^{18}$ & $\mathrm{~cm}^{-3}$ \\
Hole concentration & $5 \times 10^{18}$ & $\mathrm{~cm}^{-3}$ \\
Sheet resistance & 120 & $\Omega / \mathrm{sq}$ \\
\hline
\end{tabular}

These values give a reference for charge carrier density and mobility. For ease of printing, handling and to test the performance of two different sensor sizes, it was agreed to print $9 \times 9 \mathrm{~mm}$ and $18 \times 18 \mathrm{~mm}$ sensors, as shown in Figure 3-3.

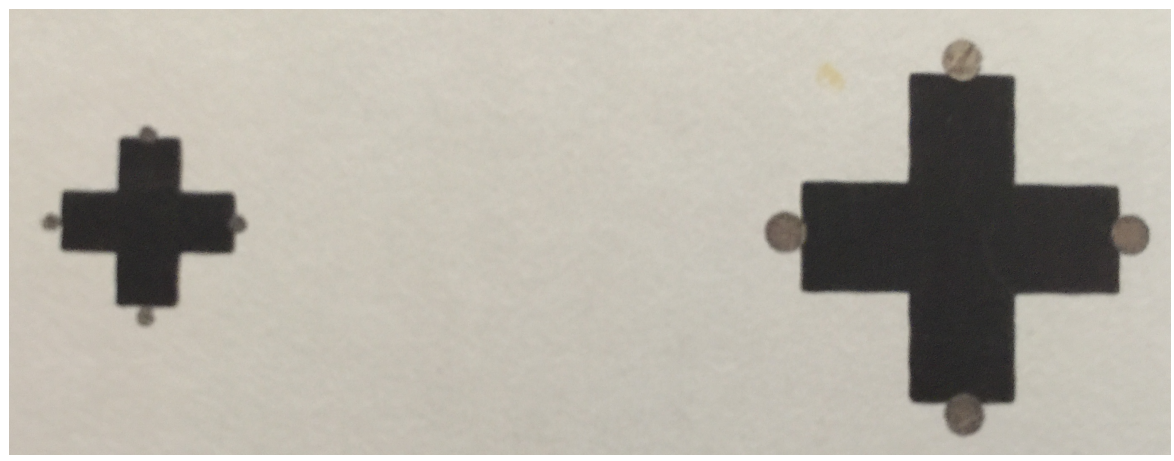

Figure 3-3. Examples of sensor printed in uncoated cardboard. Left: $9 \times 9 \mathrm{~mm}$ sensor. Right: 18x18 mm sensor. 


\subsection{First Printed Sensors}

The first Hall sensors were printed on different substrates, including paper, coated and uncoated cardboard, and polyethylene terephthalate (PET). For all cases, the resistance of the sensor was measured, and as preliminary testing, the Hall voltage was measured while placing the sensor near a strong neodymium magnet [30]. The exact magnetic field emanating from the magnet was not measured as Gaussmeters were not available and they are expensive tools. Initial sensors did not yield a measurable Hall voltage, and it was hypothesized that the thickness of the sensors hindered the Hall effect.

Recalling that the Hall voltage is inversely proportional to the thickness, an effort was made to make the sensor as thin as possible on the substrate. Further, since the sensor did not have contacts for testing, grabber test lead placement could lead to misleading results. Hence, a silver layer was placed on the edges of the sensor to distribute the test lead contact along the sensor's edge. Testing under these conditions yielded a clear jump in voltage when the neodymium magnet approached the sensor. At this point, it was decided to obtain a Helmholtz coil to have more control over the magnetic field. 


\subsection{Helmholtz Coils}

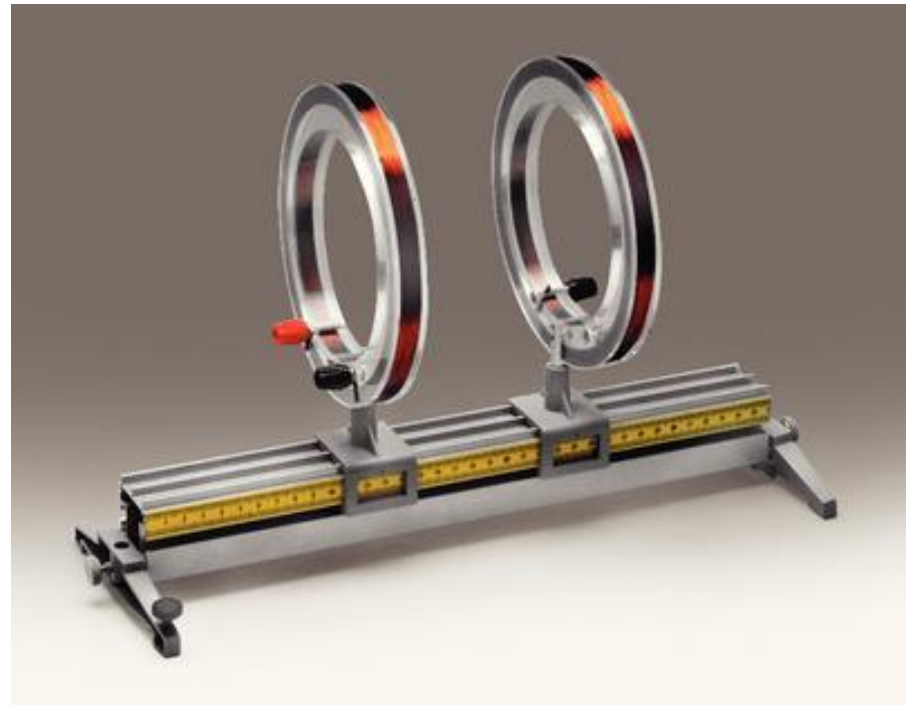

Figure 3-4. Helmholtz coils used during experiments. Each coil has 400 turns, can withstand a maximum of $1 \mathrm{~A}$ of current, and have an average coil diameter of $155 \mathrm{~mm}$. The outer diameter measures $165 \mathrm{~mm}$ and the inner diameter $145 \mathrm{~mm}$. Note that these coils are hobbyist grade [31].

Helmholtz coils (Figure 3-4) are large circular inductors whose magnetic field can be coupled to additional coils if the current is applied in the same direction in each coil. By right-hand rule, if the current flows clockwise around the coil, the inductor generates a magnetic field perpendicular to the radius of the coil, as exemplified in Figure 3-5 (a). Once coupled with other coils, the magnetic field in between the coils becomes uniform, as shown below in Figure 3-5 (b). 


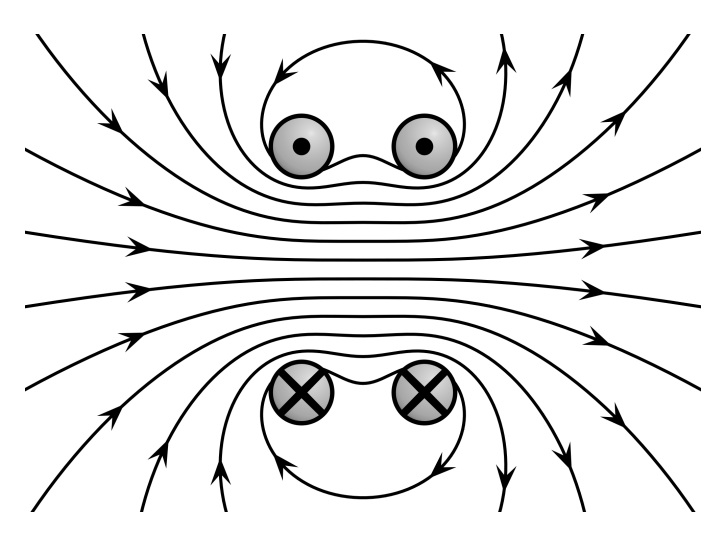

(a)

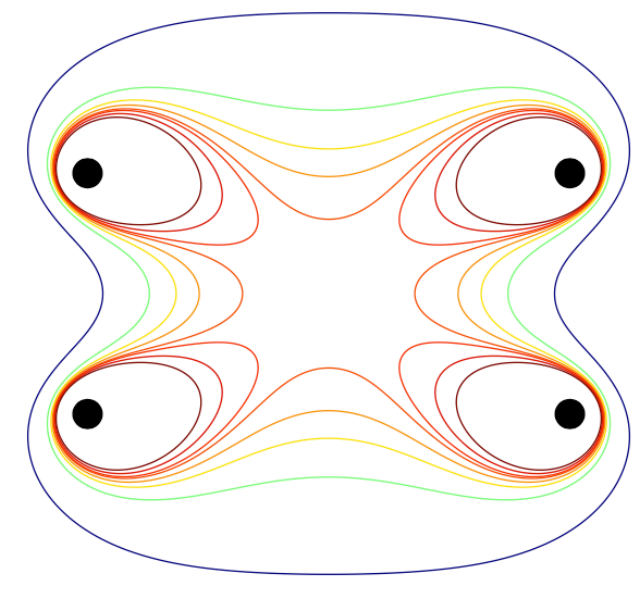

(b)

Figure 3-5. (a) Cross-section of Helmholtz coil pair and the resulting magnetic field lines with matched current direction. (b) Contours showing the uniformity of the magnitude of the magnetic field near the Helmholtz coil pair. Inside the central "octopus", the field is within $1 \%$ of its central value $\mathrm{B} 0$. The eight contours are for field magnitudes of $0.5 \mathrm{~B} 0,0.8 \mathrm{~B} 0,0.9 \mathrm{~B} 0,0.95 \mathrm{~B} 0,0.99 \mathrm{~B} 0$, $1.01 \mathrm{~B} 0,1.05 \mathrm{~B} 0$, and 1.1 B0 [32].

For two coupled coils, the magnetic field uniformity maximizes when the coils are placed half a radius away from each other. The strength of this configuration is determined with the equation below:

$$
\begin{gathered}
B\left(\frac{R}{2}\right)=\left(\frac{8}{5 \sqrt{5}}\right) \frac{\mu_{0} n I}{R}=\left(\frac{8}{5 \sqrt{5}}\right) \frac{\left(4 \pi \times \frac{10^{-7} T m}{A}\right)(400)(1 A)}{\frac{155 \times 10^{-3} m}{2}} \\
B\left(\frac{R}{2}\right)=2.32 m T
\end{gathered}
$$

where $R$ denotes the coil radius, $\mu_{0}$ represents the permeability of free space, $n$ is the number of turns and I the current through each of the coils [32]. Given equation (28), the maximum uniform attainable field strength given the coil pair used for experiments (Figure 3-4) is $2.32 \mathrm{mT}$ or $2.32 \times 10^{-7} \mathrm{~Wb} / \mathrm{cm}^{2}$. However, this is a 
theoretical value that does not account for the coil thickness. A quick COMSOL simulation with the given coil parameters shows that the approximate magnetic field strength in the center of the coils is approximately $150 \mu \mathrm{T}$, as seen below in Figure 3-7. The discrepancy in field most likely arises from the thickness of the coils not being taken into account for in (28). The Hall sensor is ready to be tested with the Helmholtz coils, but to fully characterize the sensor the Van der Pauw method was used.

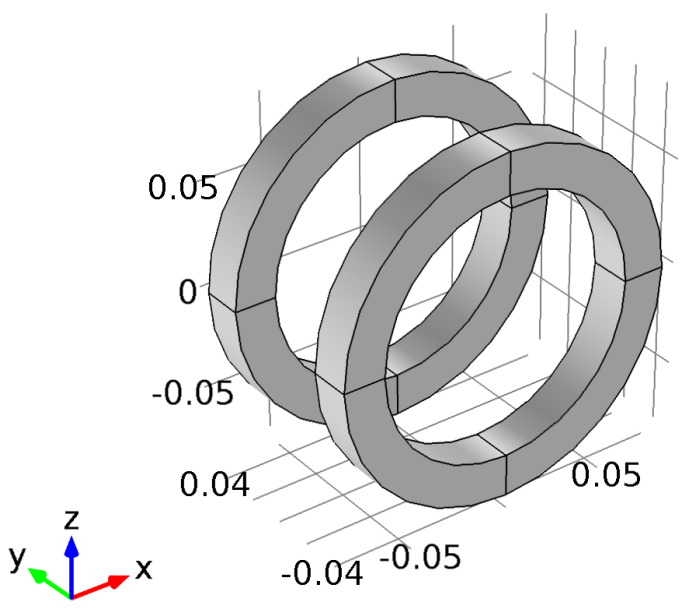

Figure 3-6. COMSOL Helmholtz coil pair geometry used. Average coil diameter of $155 \mathrm{~mm}$. The outer diameter measures $165 \mathrm{~mm}$ and the inner diameter $145 \mathrm{~mm}$. To maximize the uniformity of magnetic field, the coils are half a radius away from each other, i.e. $38.75 \mathrm{~mm}$. 


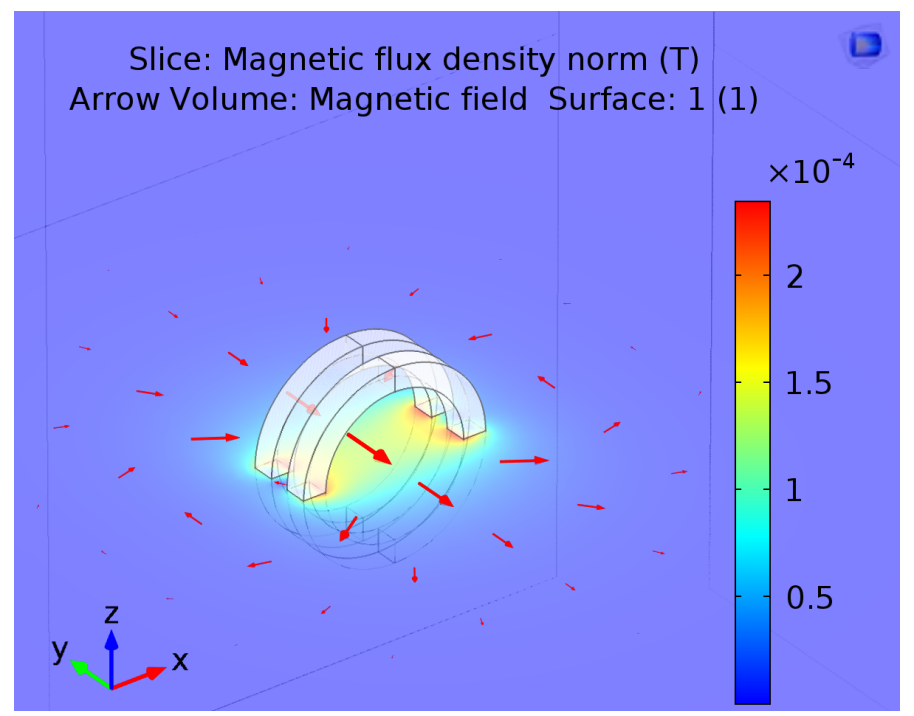

Figure 3-7. COMSOL Helmholtz coil pair simulation using parameters from the coils used for experiments. The approximate field strength in the middle of the coils is $150 \mu \mathrm{T}$.

\subsection{Van der Pauw Method}

The Van der Pauw method is a common technique used to determine resistivity and the Hall coefficient of different materials. The following conditions must be met in order to perform accurate measurements for a sample:

1. The shape of the sample must be flat and possess uniform thickness.

2. The sample must not have isolated holes within the sample.

3. The sample must be homogeneous and isotropic.

4. The contacts must be at the edges of the sample.

5. The contact area has to at least be an order of magnitude less than the entire sample area. 
The conditions above suggest that the sensors with silver contacts will not yield accurate measurements. Additionally, sensors were printed in acrylic and glass to further improve smoothness of the sensor, and hence improve Hall response.

\subsubsection{Resistivity and Sheet Resistance}

To calculate the resistivity of the sample, each of the four terminals of the Hall sensor were numbered in counter-clockwise direction, as shown below, in Figure 3-8.

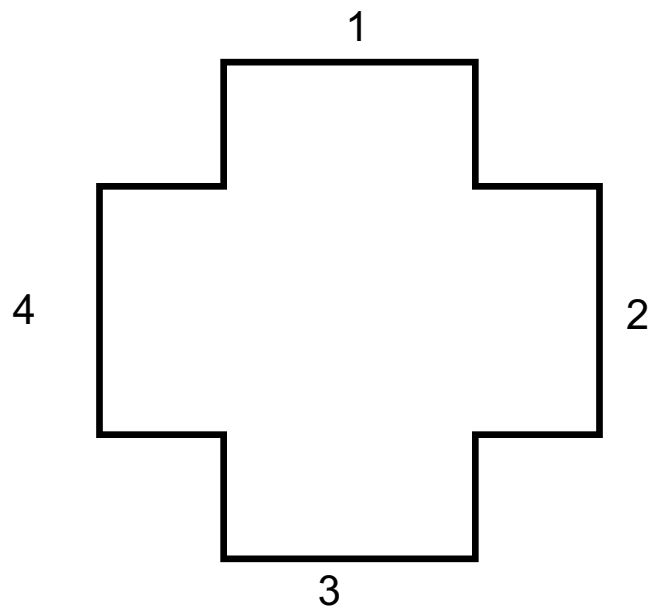

Figure 3-8. Diagram showing the terminal configuration numbering convention for Van der Pauw method method and calculations.

Resistance was calculated by applying a current through two adjacent terminals, while measuring the voltage in the other two terminals. The results are shown below in Table 3-2. The percent difference between reciprocal configurations is less than $3 \%$ in all cases, which indicates a good accuracy in measurements. The Van der Pauw formula [33] states 


$$
e^{-\pi R_{v} / R_{S}}+e^{-\pi R_{h} / R_{S}}=1
$$

where, $R_{v}$ and $R_{h}$ represent vertical and horizontal device resistance, respectively, and are calculated as such,

$$
\begin{aligned}
& R_{v}=\frac{R_{12,34}+R_{34,12}+R_{21,43}+R_{14,32}}{4} \\
& R_{h}=\frac{R_{23,41}+R_{41,23}+R_{32,14}+R_{14,32}}{4}
\end{aligned}
$$

The notation $R_{12,34}$ refers to terminal connections in the Hall sensor for the measurement in question (i.e., $R_{12,34}=V_{34} / l_{12}$ ). Sheet resistance $R_{s}$ is ideally calculated when $R_{v}$ and $R_{h}$ are equal. However, since this was not the case, $R_{a v g}$ was used instead. Hence, solving for $\mathrm{R}_{\mathrm{s}}$ in (29),

$$
R_{S}=\frac{\pi R_{\text {avg }}}{\ln (2)}
$$

Table 3-2. Resistance measurements using the Van der Pauw method. The first column, Configuration, refers to the terminals of the Hall sensor. For example, configuration 12,34 has a current being sinked through terminal 1 and 2 , while measuring voltages from 3 and 4 . In all cases, the current applied was $1 \mathrm{~mA}$. The last column refers to the calculated percent difference between each reciprocal terminal configurations (i.e. 12,34 and 34,12 are a pair of reicprocal terminal configurations).

\begin{tabular}{ccccc}
\hline Config. & $\mathrm{I}(\mathrm{mA})$ & $\mathrm{V}(\mathrm{mV})$ & $\mathrm{R}(\Omega)$ & $\begin{array}{c}\text { \%Diff. Rev. } \\
\text { Polarity }\end{array}$ \\
\hline 12,34 & 1 & -22.916 & 22.916 & -0.678 \\
34,12 & 1 & -23.072 & 23.072 & \\
\hline 21,43 & 1 & -22.14 & 22.14 & -2.198 \\
43,21 & 1 & -22.632 & 22.632 & \\
\hline 23,41 & 1 & -23.926 & 23.926 & \multirow{2}{*}{1.215} \\
41,23 & 1 & -23.637 & 23.637 & \\
\hline 32,14 & 1 & -23.825 & 23.825 & 2.001 \\
14,32 & 1 & -23.353 & 23.353 & \\
\hline
\end{tabular}


Results from Table 3-2 yield a sheet resistance of $105.09 \Omega / \mathrm{sq}$, which yields a $-12.44 \%$ difference when compared to the datasheet specification for the carbon ink of $120 \Omega / \mathrm{sq}$. To calculate further material electrical properties, the Helmholtz coil pair was required.

\subsubsection{Hall Voltage, Sheet Density and Majority Carrier Mobility}

Using the Helmholtz coil pair, the Hall sensor was mounted as shown in Figure 3-9. (a) Helmholtz coil test setup. The triple power supply provides power to each of the coils and the biasing current for the sensor. (b) Sensor placement on the Helmholtz coil. As previous Hall sensor simulations, a constant biasing current of $1 \mathrm{~mA}$ was applied horizontally, while measuring the potential difference vertically while in the presence of first positive and then negative magnetic fields. The difference between these two voltages for this specific configuration was noted. Afterwards, the procedure was repeated with vertical current and a horizontal voltage measurement. The results are shown below, in Table 3-3. Hall voltage measurements under the influence of magnetic fields. Taking the average of the voltage differences for each configuration yields a Hall voltage of $14 \mu \mathrm{V}$. Due to the wide range in measured potential differences from Table 3-3. Hall voltage measurements under the influence of magnetic fields. The first column refers to the terminals which were used for the biasing current. The second and third column refer to positive and negative fields applied, respectively. The last column represents the difference between columns two and three, which represents the resulting Hall voltage for that particular configuration., the measured Hall voltage 
should not be considered as conclusive evidence that the printed sensor works.

Regardless, majority carrier mobility and density was still calculated.

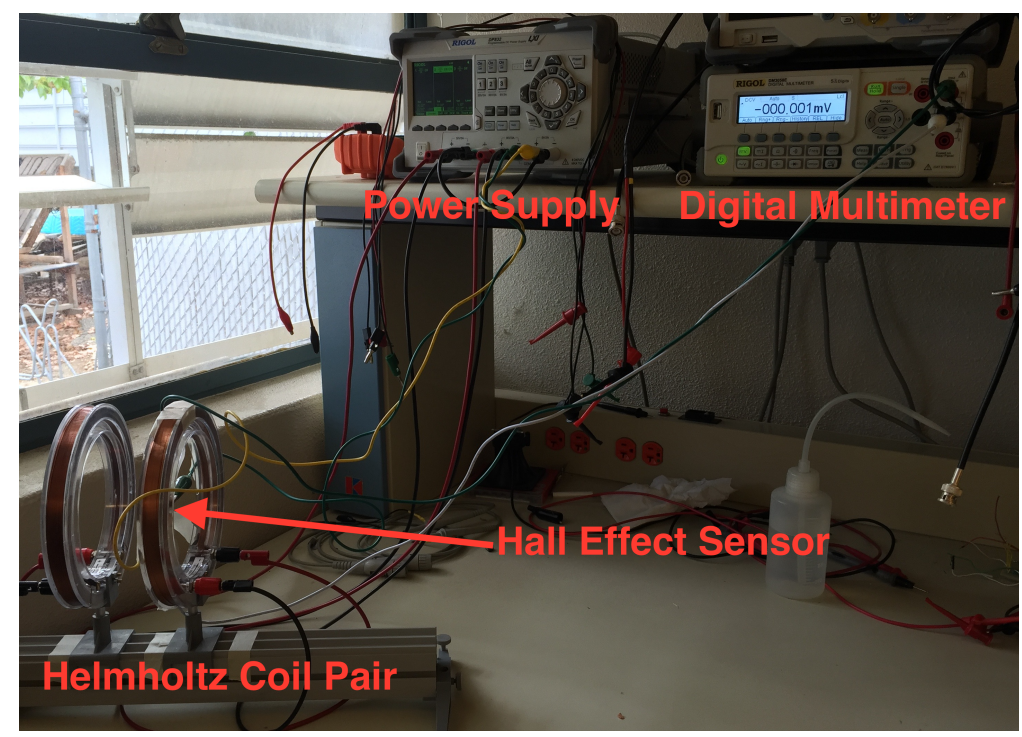

(a)

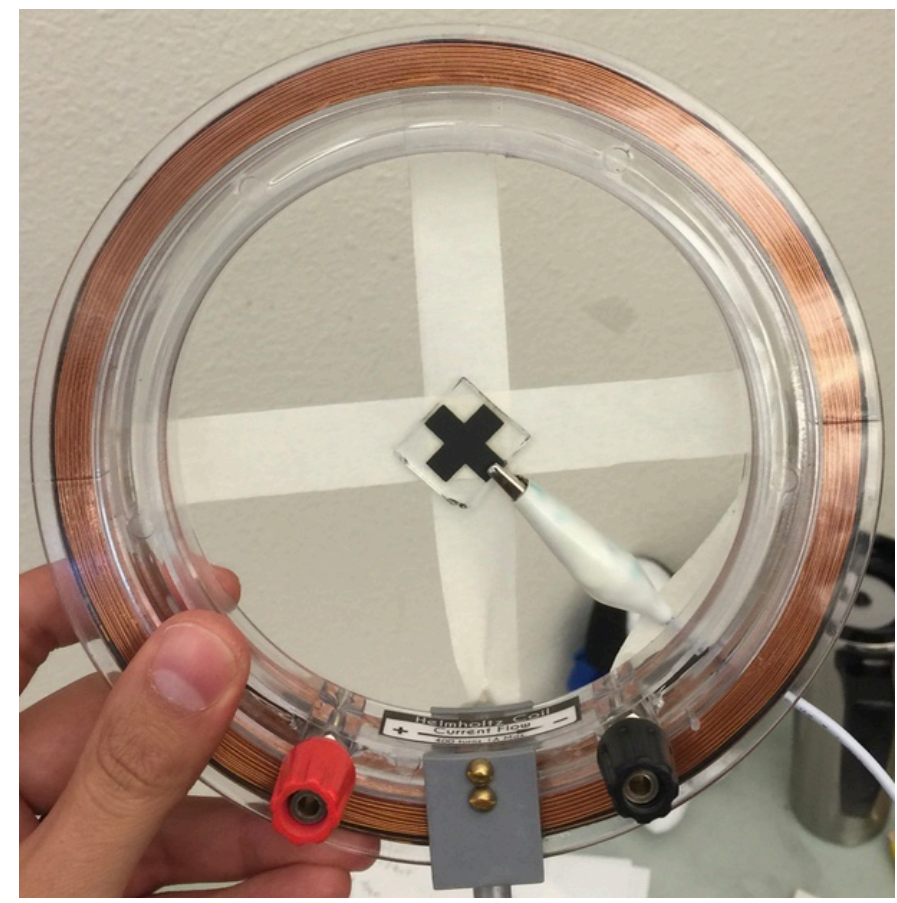

(b)

Figure 3-9. (a) Helmholtz coil test setup. The triple power supply provides power to each of the coils and the biasing current for the sensor. (b) Sensor placement on the Helmholtz coil. 
Table 3-3. Hall voltage measurements under the influence of magnetic fields. The first column refers to the terminals which were used for the biasing current. The second and third column refer to positive and negative fields applied, respectively. The last column represents the difference between columns two and three, which represents the resulting Hall voltage for that particular configuration.

\begin{tabular}{cccc}
\hline Config. & $\mathrm{P}(\mu \mathrm{V})$ & $\mathrm{N}(\mu \mathrm{V})$ & $\mathrm{VH}(\mu \mathrm{V})$ \\
\hline 13 & 418 & 391 & 27 \\
24 & 351 & 355 & -4 \\
31 & -314 & -354 & 40 \\
42 & -310 & -356 & 46 \\
\hline
\end{tabular}

The sheet charge carrier density, was then calculated from the Hall voltage equation (5)

$$
n_{s}=\frac{I B}{\mathrm{q}\left|\mathrm{V}_{H}\right|}=\frac{(1 \mathrm{~mA})\left(1.50 \times 10^{-8} \frac{\mathrm{Wb}}{\mathrm{cm}^{2}}\right)}{\left(1.602 \times 10^{-19} \mathrm{C}\right)|13.6 \mu V|}=6.87 \times 10^{12} \mathrm{~cm}^{-2}
$$

which yields a charge carrier density of $3.82 \times 10^{17} \mathrm{~cm}^{-3}$ since the measured sensor thickness was $18 \mu \mathrm{m}$. Further, treating the carbon ink as a semiconductor and using the conductivity equation (7), the majority carrier mobility is calculated as such,

$$
\begin{gathered}
\mu_{m}=\frac{1}{\mathrm{qn}_{s} R_{s}}=\frac{1}{\left(1.602 \times 10^{-19} \mathrm{C}\right)\left(6.87 \times 10^{12} \mathrm{~cm}^{-2}\right)(105.09 \Omega)} \\
\mu_{m}=8644 \frac{\mathrm{cm}^{2}}{V s}
\end{gathered}
$$

\subsubsection{Analysis of the Van der Pauw Method}

It was hypothesized that the conductive ink would exhibit similar properties to graphite. As seen in Table 3-4, there is an evident difference between graphite and 
the conductive ink. The sheet resistance was the experimental result that most closely resembles the estimated value. Considering that sheet resistance was the only value obtained directly from the manufacturer's datasheet [26], a small divergence from the manufacturer's ink curing instructions might yield slightly different properties. In this case, the manufacturer states that the sheet resistance of $120 \Omega / s q$ were measured at $25 \mu \mathrm{m}$. However, the printed sensor measured $18 \mu \mathrm{m}$ in thickness. This means that this $13 \%$ difference might have been caused by differences in thicknesses.

Despite the great percent difference between graphite and carbon ink material properties, the experimental data is still on the same order of magnitude compared to other elements, such as arsenic, phosphorous and boron [34, Ch. 2]. Nonetheless, due to the great discrepancy in measured Hall voltages, one should remain skeptical to the printed ink's effectiveness as a Hall sensor.

Table 3-4. Estimated and experimental results for the Van der Pauw method, assuming the ink approximately behaves like graphite [29]. ${ }^{\dagger}$ Sheet resistance was obtained from manufacturer's datasheet [26].

\begin{tabular}{ccccc}
\hline Property & Estimated & Experimental & Unit & $\%$ Diff. \\
& Value & Results & & \\
\hline Hole mobility & $15 \times 10^{3}$ & 8644 & $\frac{\mathrm{cm}^{2}}{V s}$ & $53.8 \%$ \\
Hole concentration & $5 \times 10^{18}$ & $3.82 \times 10^{17}$ & $\mathrm{~cm}^{-3}$ & $170 \%$ \\
Sheet resistance $^{\dagger}$ & 120 & 105 & $\Omega / \mathrm{sq}$ & $13 \%$ \\
\hline
\end{tabular}


Hall voltage measurements were difficult to obtain. The sensor's offset seemed to constantly drift positively or negatively at seemingly random intervals, as exemplified in Figure 3-10. This behavior is most likely temperature- and/or stressdependent [3], [35]. It was further noticed that the alligator clips used for probing the sensor were being extremely destructive to the sensor, as seen in Figure 3-11. Any minor vibration might have caused a scratch, which then translates into a change in offset. These issues might have introduced inaccuracies in measurements, and hence these results do not conclusively prove that the printed Hall sensor can sense magnetic fields. A possible solution might be to increase the magnetic field strength and obtain a Hall response larger than temperature drift. Additionally, mounting and fixing in place the sensor, as well as the alligator clips to a hard surface might increase control on the clips, reduce movement and reduce stress caused on the sensor.

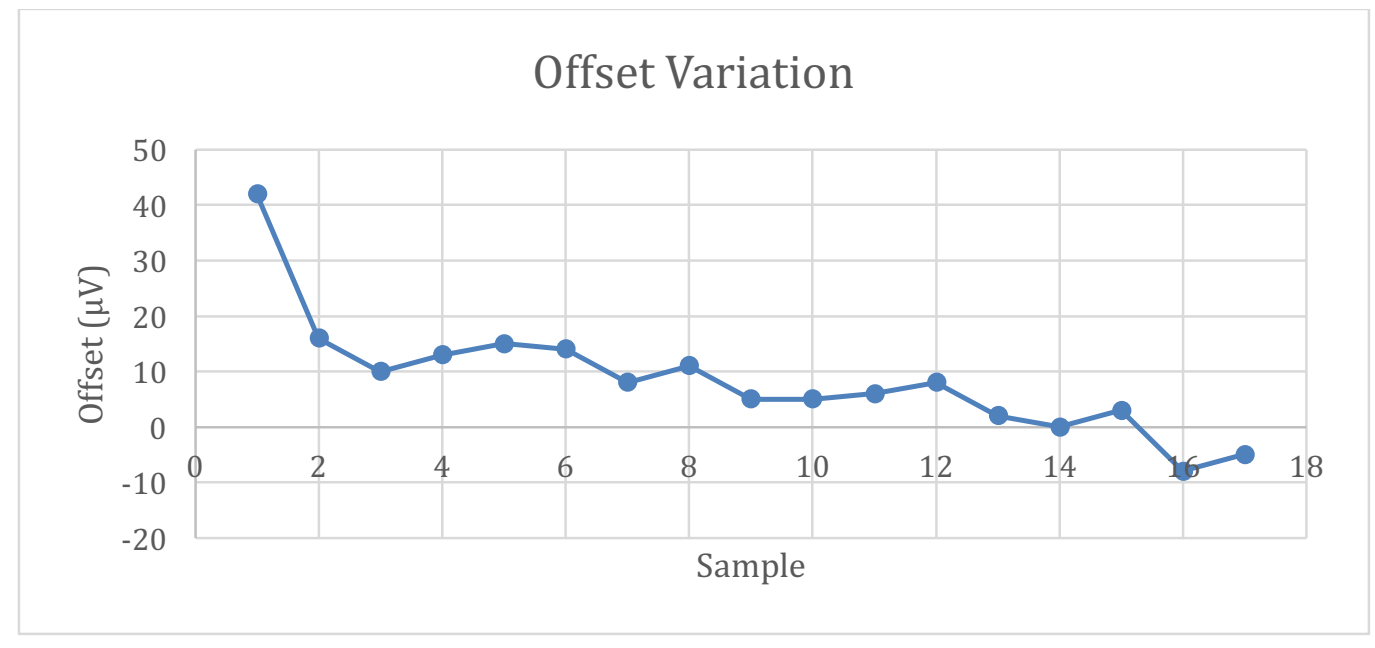

Figure 3-10. Offset sampling over time. The sample number corresponds to the time the offset was sampled, i.e. sample 1 was the first offset measurement and 15 the last. 


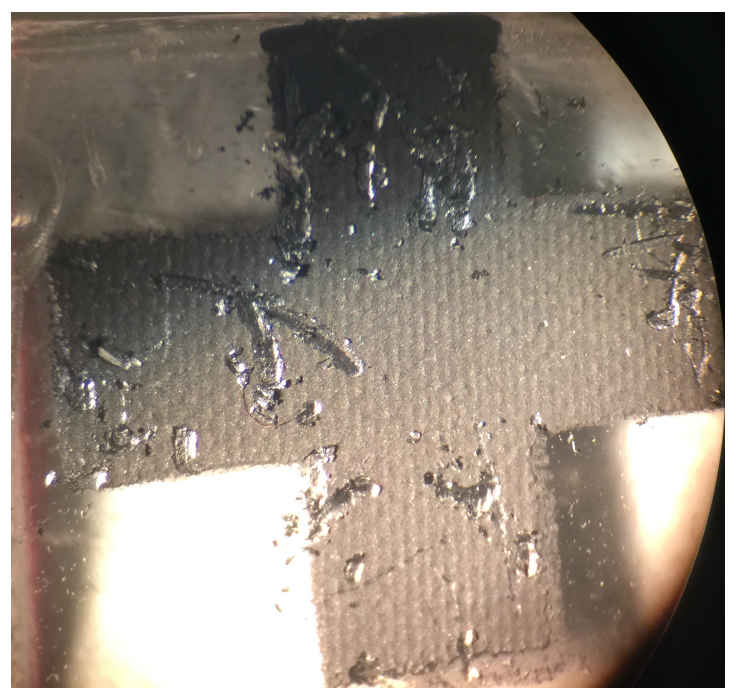

Figure 3-11. Close-up from one of the sensors printed on a glass substrate. The scratches come from probing and handling the sensor. Note that the scratches did not completely remove the ink from the glass substrate.

The Helmholtz coil, although it introduced more control to the magnetic field affecting the sensor, the strength of such field was limited as the Helmholtz coil simulation and hand calculations (28) showed. Hence, such small measurements were not attainable with the equipment available.

\subsection{Neodymium Magnet Pair Test}

A stronger field should, theoretically, show conclusively if the sensor responds or not to magnetic fields. Hence, a pair of 1" neodymium cube magnets [36] where used to introduce the printed sensor to a strong and relatively even magnetic field. 


\subsubsection{Test Fixture Setup}

In the absence of a Gaussmeter to measure magnetic fields, one was built according [37]. P/N A1302, a continuous-time ratiometric linear Hall effect sensor IC, has a sensitivity of approximately $1.3 \mathrm{mV} / \mathrm{G}$. Even though the magnets might overpower the constructed Gaussmeter, the ability to measure magnetic fields will greatly help for measuring the effects of weaker magnetic fields. The Gaussmeter was shunted as close as physically possible to the printed sensor to measure the magnetic field that the sensor experiences during measurements.

The sensor was fixed in one spot with the help of a clamp to ensure that the sensor was not being affected by subtle test lead movements, and hence introduce changes in voltage measurements. When the magnetic field was needed for testing, the two cube magnets were placed 5 or $7 \mathrm{~cm}$ away from the sensor in opposite directions, depending on the desired field strength. A diagram and a picture of the setup are shown below, in Figure 3-12 and Figure 3-13. A visualization for the expected magnetic field from the two magnets is shown in Figure 3-14. 


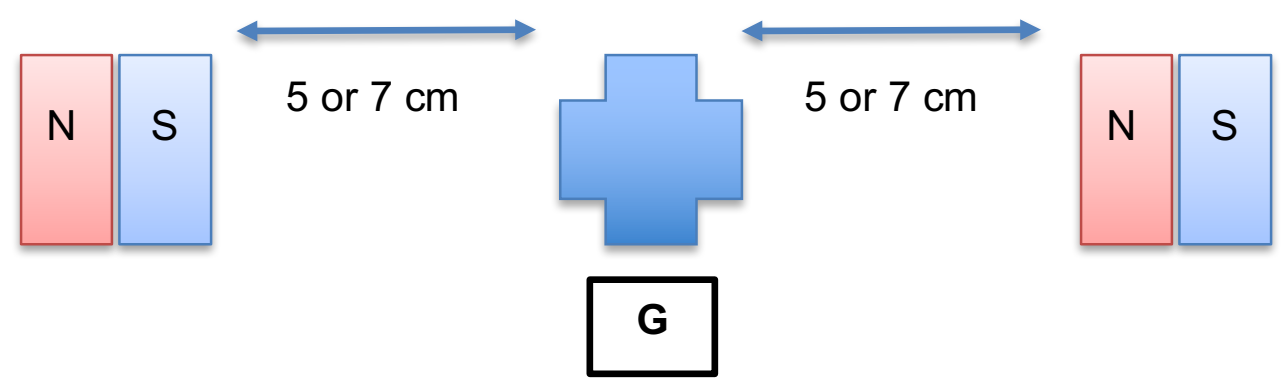

Figure 3-12. Magnet pair test setup. If the measurement required a magnetic field, the magnets were placed 5 or $7 \mathrm{~cm}$ away from the sensor, depending on desired field strength. If no magnetic field was required, then the magnets were removed. The sensor is connected to horizontal current biasing and vertical voltage measurement or vice-versa. The $\mathrm{G}$ represents the Gaussmeter placement.

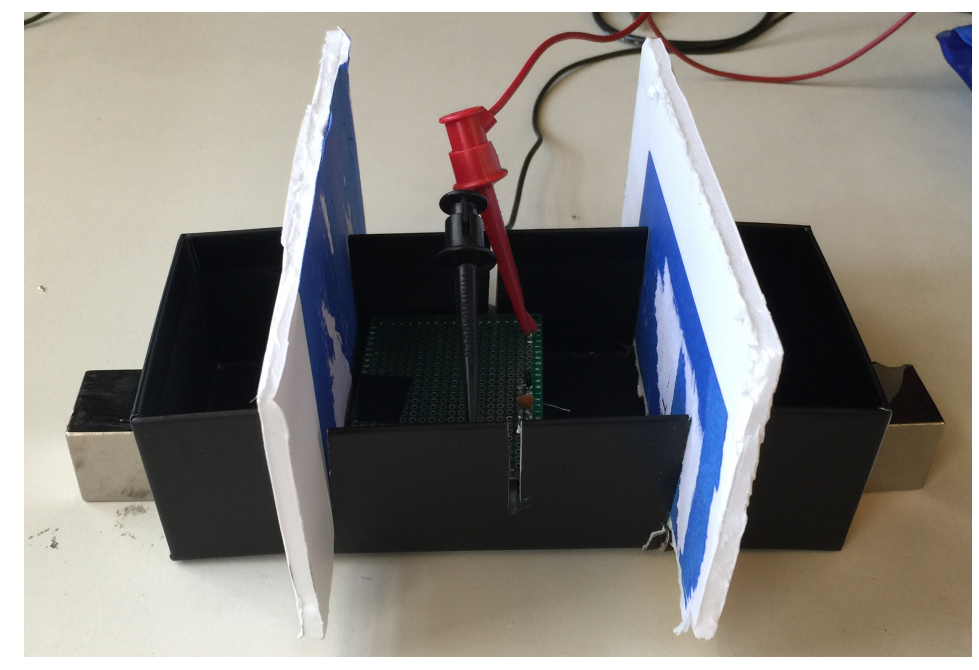

Figure 3-13. Magnet pair test fixture setup. The silver cubes are the magnets. The device in the middle is the constructed Gaussmeter. The white cutouts serve as the $5 \mathrm{~cm}$ markers and also prevent the magnets from collapsing onto each other when placed at this spot. Not shown is the sensor base, which fits in the middle slot. 


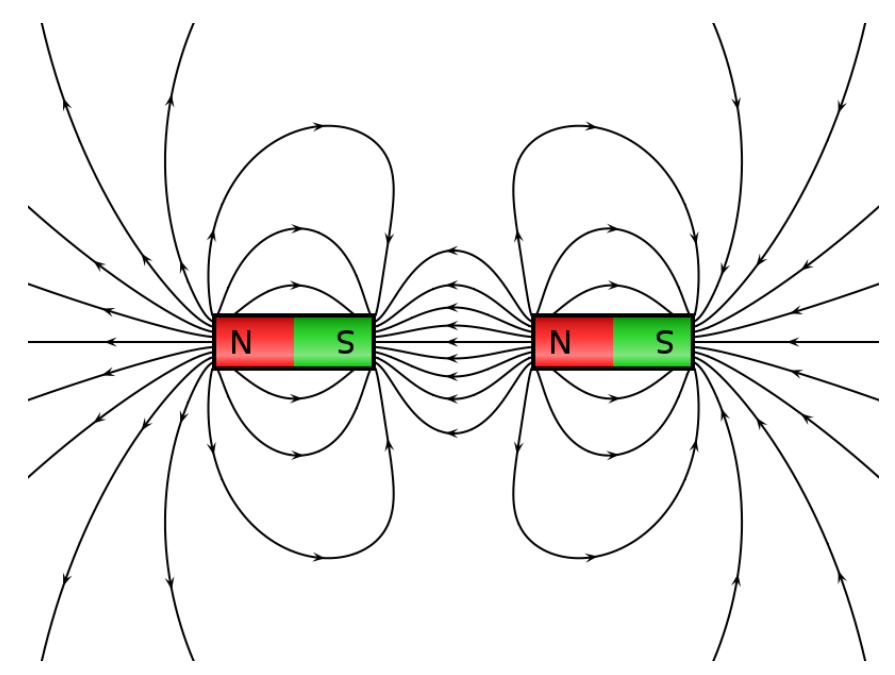

Figure 3-14. Magnetic field lines visualization for an attracting pair of magnets [38].

\subsubsection{Testing and Results}

Several printed sensor variants were tested, and they returned the data presented in this section. Since the previous Van der Pauw measurement took at least 4 hours to complete due to drifting offset voltages, it was decided to attempt a few preliminary tests before attempting the Van der Pauw method once again.

\subsubsection{Preliminary Testing}

First, the $18 \mathrm{~mm}$ Glass sensor was put through a preliminary test of shifting the cube magnets around the setup in Figure 3-13. The linearity of the results seemed promising for for future tests. Hence, more data points were taken in the second preliminary test, as shown in Figure 4-3. However, the sensor most likely was disturbed during testing, which probably caused a completely different kind of response. 


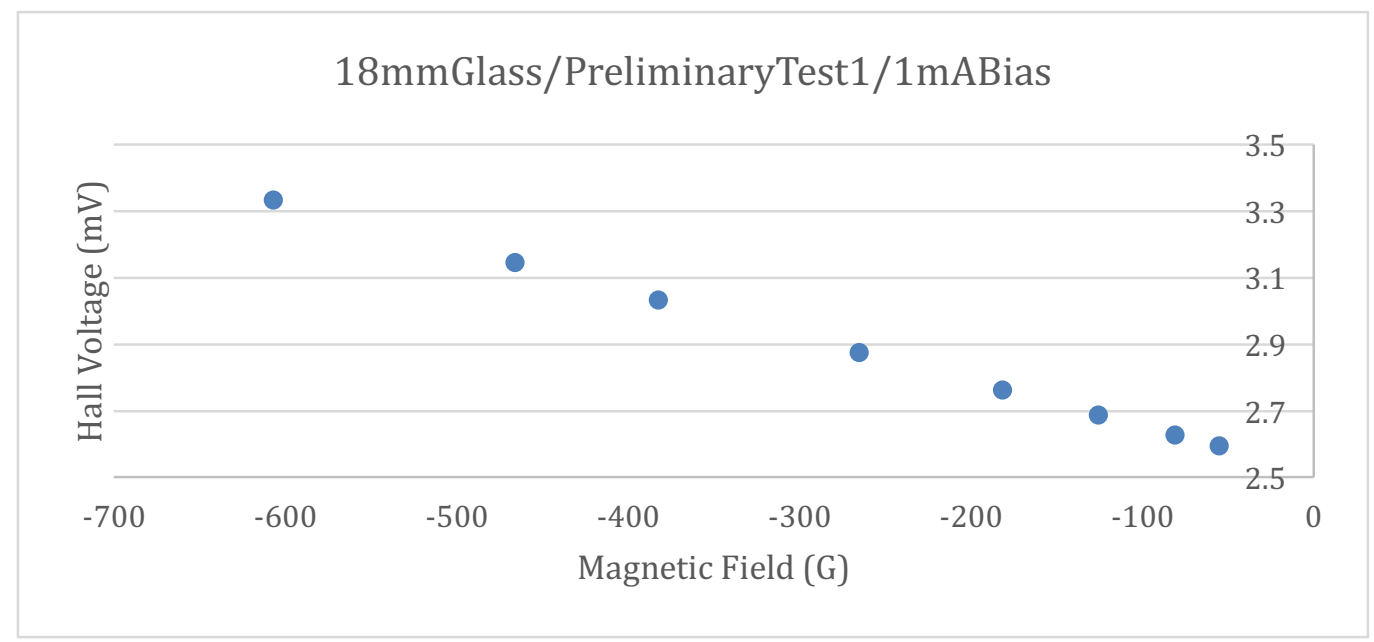

Figure 3-15. Preliminary test for $18 \mathrm{~mm}$ glass sensor biased at $1 \mathrm{~mA}$. The highly linear responses showed promising results for future in-depth tests.

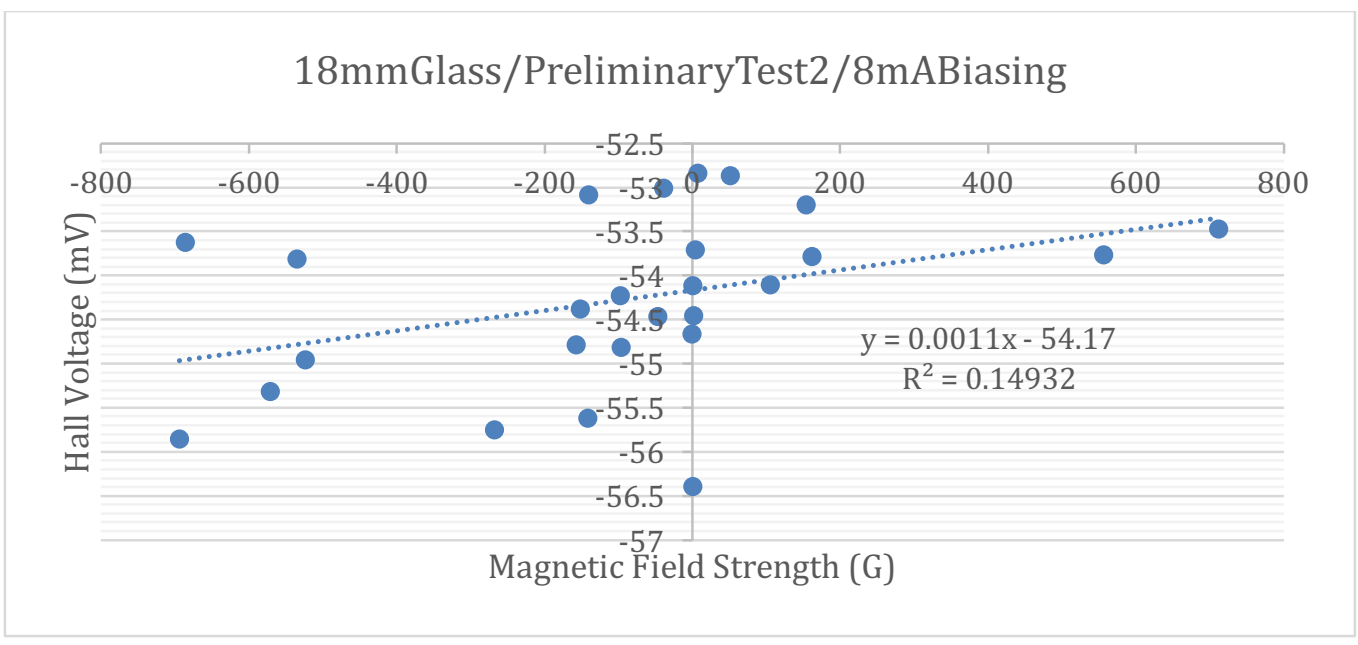

Figure 3-16. Preliminary test 2 . Results from holding the biasing current at $8 \mathrm{~mA}$ and modifying the magnetic field strength by modifying the positioning of both cube magnets. The sensor yielded completely different offset voltage when compared to Figure 3-15. Similarly, the voltage/field slope flipped polarity. 


\subsubsection{Offset Reduction via Oversized Contacts}

Current literature suggests that using contacts that cover the entirety of the sensors' edges are better for eliminating offset issues [39]. This might occur because the contact "averages" the voltage perceived at the edges of the sensor as opposed to multiple-point contacts from alligator clips. Hence, diverse bonding techniques were attempted in the hopes to place highly conductive material on the edges of the sensor to diminish offset issues. One of the attempts consisted of attaching copper tape to the edges of the sensors, and soldering over the tape to attempt to fuse the ink to copper tape. This attempt failed since the ink evaporated from the high temperature. Another attempt involved applying conductive gel [40], similar to the type used for electrocardiogram (ECG) electrodes, to the edges of the sensors and bonding wires to the gel. Unfortunately, wires do not bond with this adhesive gel. Alligator clips were placed on the remaining adhesive conductive gel to try to distribute the clips' contacts with the sensor but the sensor's response was still very unstable. Figure 3-17 displays a picture with some of the attempts just mentioned.

The most stable solution attempted thus far involves placing a thin layer of silver ink on the sensor edges while printing on a PET film. The silver ink is then sintered, to connect the carbon and silver inks. Figure 3-18 shows a picture of the silver contacts sensor. Nonetheless, since the sensor is printed on a flexible film, it had to be mounted to a hard and stable surface to prevent any offsets from reappearing during testing. 


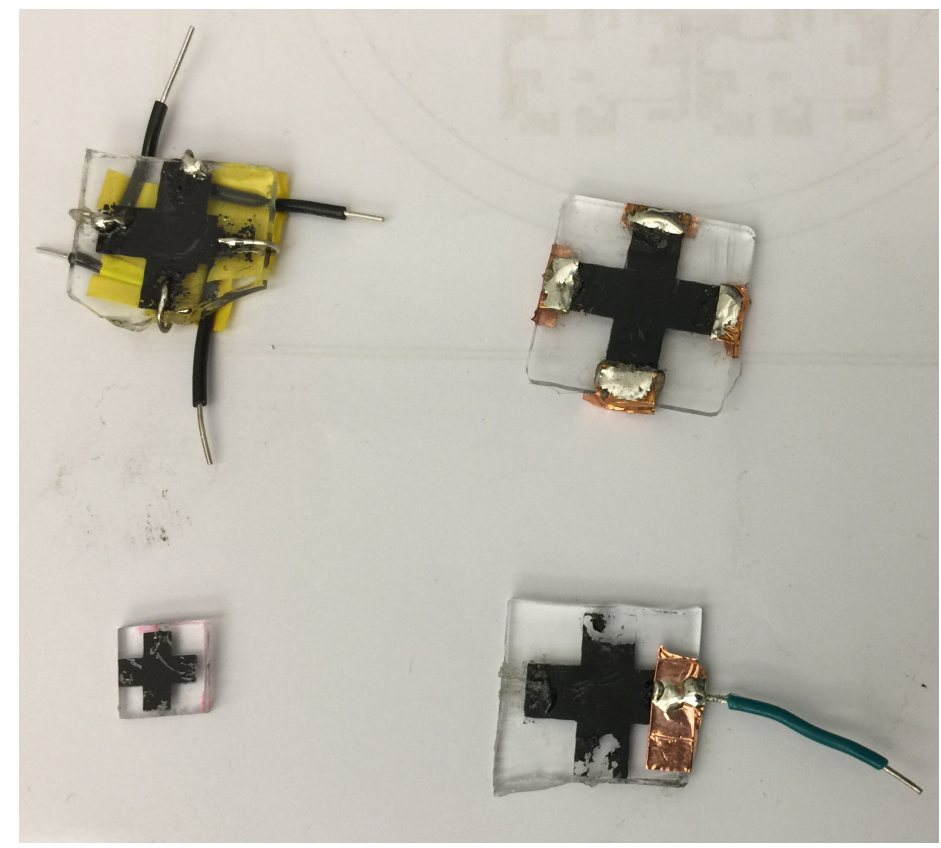

Figure 3-17. Picture showing damaged sensors from metal contact bonding attempts.

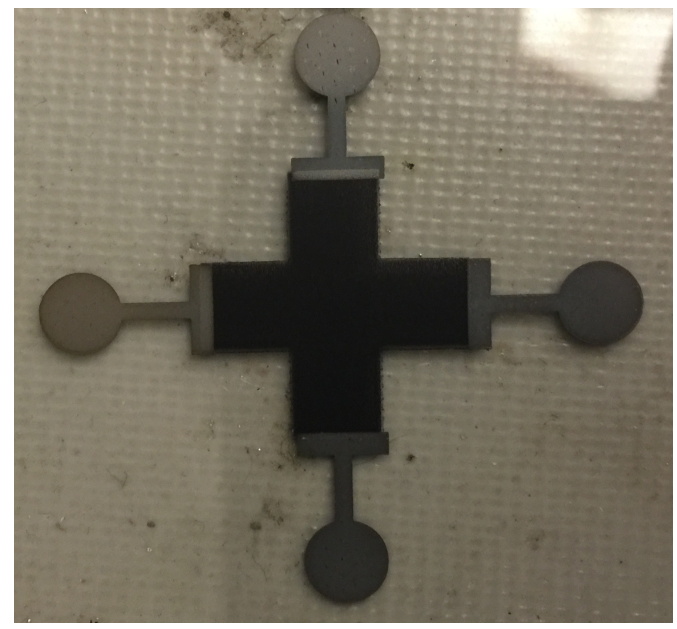

Figure 3-18. Sensor on PET film with silver contacts. This device was the most effective at eliminating the offset drift. 


\subsubsection{Sensors with Silver Contacts}

The next device tested was an $18 \mathrm{~mm}$ sensor with silver contacts biased at 100 $\mathrm{mV}$. A very low voltage was used to try to eliminate random offset fluctuations. Again, the sensor was placed in the text fixture while the magnets where moved around to produce diverse magnetic fields. The data collected is shown in Figure 3-19. Due to the roughly even symmetry in Hall voltage, it was assumed that the sensor was still malfunctioning. Hence it was decided to test a new device.

The last device tested was a $9 \mathrm{~mm}$ sensor with silver contacts. The data collected from this sensor is shown in Figure 3-20. This device also showed the rough even symmetry in Hall response. Since this device was outputting relatively consistent values, a total of 50 samples were obtained from this test alone. Further, due to the consistent response, it was decided to try the Van der Pauw method once again.

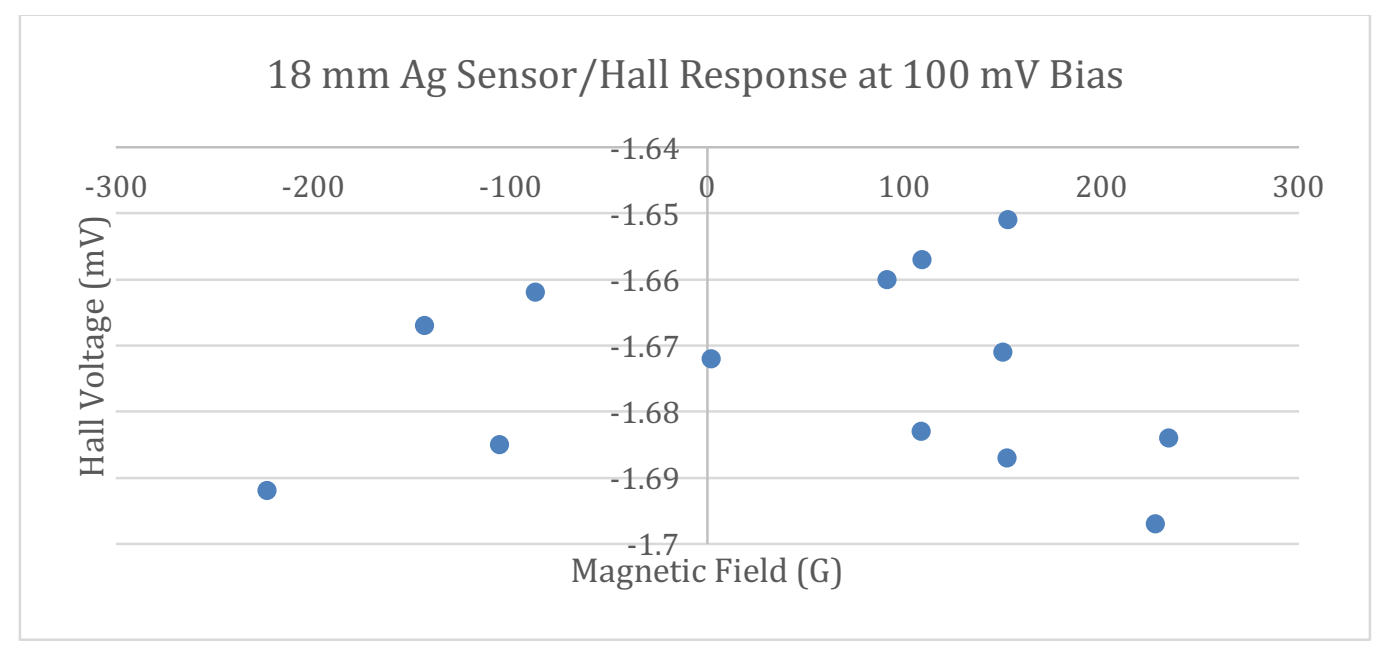

Figure 3-19. $18 \mathrm{~mm}$ sensor with silver contacts tested at $100 \mathrm{mV}$ bias. 


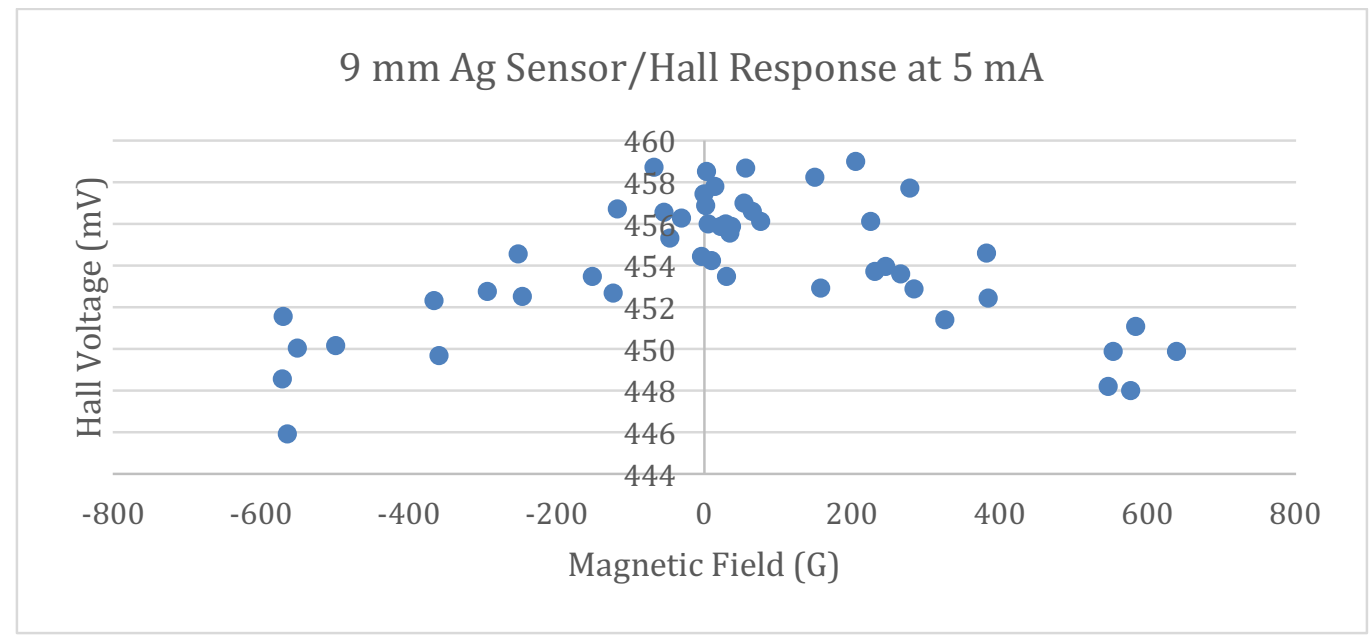

Figure 3-20. $9 \mathrm{~mm}$ sensor with silver contacts tested at $5 \mathrm{~mA}$ bias.

\subsubsection{Results from the Van der Pauw Method Revisited}

The Van der Pauw method, which was previously detailed in section 3.5, was attempted with the $9 \mathrm{~mm}$ sensor with silver contacts. The Hall voltage spinning measurements are shown in Table 3-5. These results were significantly more stable than Helmholtz results without silver contacts. Further, results from the Van der Pauw method are summarized in Table 3-6. For comparison, this table also contains previous results from the Helmholtz-Van der Pauw method calculations.

Table 3-5. Hall voltage data for the Van der Pauw method. The calculated Hall voltage from this table is $98 \mu \mathrm{V}$ at $527 \mathrm{G}$.

\begin{tabular}{cccc}
\hline Config. & $\mathrm{P}(\mathrm{mV})$ & $\mathrm{N}(\mathrm{mV})$ & $\mathrm{VH}(\mathrm{mV})$ \\
\hline 13 & 150.092 & 156.806 & -6.714 \\
31 & -149.982 & -157.476 & 7.494 \\
24 & 138.361 & 132.64 & 5.721 \\
42 & -145.978 & -140.262 & -5.716 \\
\hline
\end{tabular}


Table 3-6. Comparison of Van der Pauw method results from using the Helmholtz coil pair and the neodymium magnets with silver contacts on the sensors. $15 \mu \mathrm{m}$ is the assumed PET sensor thickness.

\begin{tabular}{ccccc}
\hline Property & $\begin{array}{c}\text { Estimated } \\
\text { Value }\end{array}$ & $\begin{array}{c}\text { Helmholtz } \\
\text { Results }\end{array}$ & $\begin{array}{c}\text { Neodymium/Ag } \\
\text { Results }\end{array}$ & Unit \\
\hline Hole mobility & $15 \times 10^{3}$ & 8644 & 8.86 & $\frac{\mathrm{cm}^{2}}{\mathrm{Vs}}$ \\
$\begin{array}{c}\text { Hole } \\
\text { concentration } \\
\begin{array}{c}\text { Sheet } \\
\text { resistance }\end{array}\end{array}$ & $5 \times 10^{18}$ & $3.82 \times 10^{17}$ & $4.47 \times 10^{18}$ & $\mathrm{~cm}^{-3}$ \\
\hline
\end{tabular}

The new hole mobility value is still significantly different than graphite. In the other hand, the updated hole concentration was significantly closer to graphite's concentration. Regardless, these Hall voltage measurements were considerably more consistent than the ones obtained from the Helmholtz coil pair experiment. This implies that the silver contacts indeed diminish the offset in the Hall sensor.

To test if this newly collected data has any relevance, the calculated material properties from this latest Van der Pauw measurements were imported into COMSOL. The simulation model cannot replicate the uncommon Hall voltage shape (Figure 3-20) from this printed sensor. However, the model should be able to somewhat approach the Van der Pauw method Hall voltage calculated $(98 \mu \mathrm{V}$ at $527 \mathrm{G}$ or $52.7 \mathrm{mT}$ ). Simulation results are shown in Figure 3-21. 


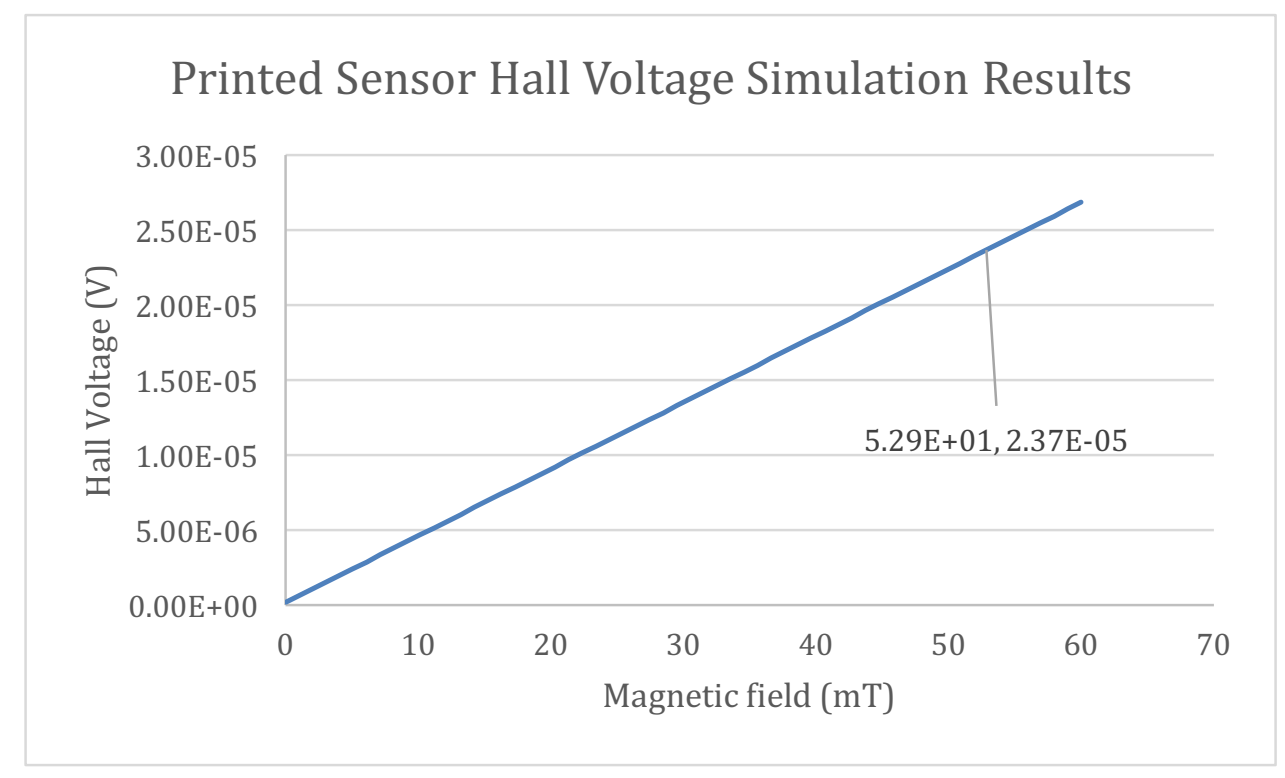

Figure 3-21. Simulation based on results obtained from the neodymium Van der Pauw method test.

The simulated data shows that at $52.9 \mathrm{mT}$, the expected Hall voltage should measure $23.7 \mu \mathrm{V}$. The measured voltage, although in the same order of magnitude, is considerably different than the simulated value. The simulation results indicate that data collected from the Van der Pauw method was still not very accurate, even after diminishing offset with the silver contacts and enhancing the field strength.

\subsection{Future Work}

As previously mentioned, the Hall sensors are sensitive to temperature. A possible solution is presented in [3], which claims that temperature drift can be fixed by the means of a temperature compensation circuit that heats up the hall device by 
injecting a large enough current into it. However, this solution seems to have been obsoleted by a method that involves conditioning the Hall voltage via a two-phase spinning current, which dynamically eliminates the offset in the adder, since the sample/holds (S/Hs) keep the Hall voltage polarity, but reverse polarity for offsets [39]. The low-frequency content from the adder contains the Hall signal, which is then passed through a filter to eliminate high-frequency noise. The block diagram for this system is shown in Figure 3-22. However, this method cannot be realized unless a stable contact with the sensor is made. Further, this method might not allow to determine material properties of the sensor.

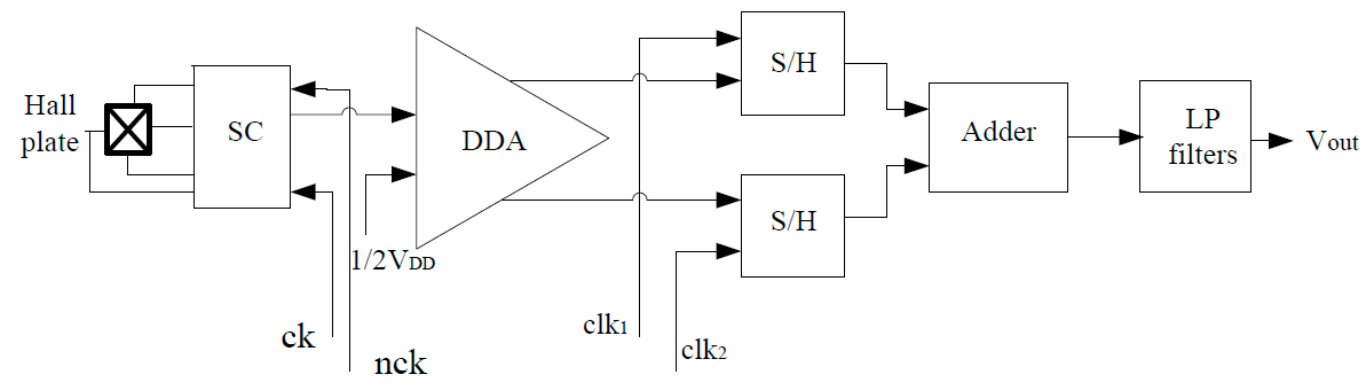

Figure 3-22. Block diagram of CMOS integrated linear Hall sensory microsystem [39].

Throughout experimentation with the conductive ink sensor, it was observed that the alligator clips introduce permanent sensor behavioral changes by puncturing the substrate or scratching the ink; therefore, these clips are not suited for probing the ink directly. The most stable known solution to this problem are the silver contacts, which have experimentally shown to diminish sensor offset significantly. To further improve sensor testing and characterization, a more robust Helmholtz coil can improve results, especially if it reaches magnetic fields in the order of 
100 mT. Additionally, a laboratory-grade Gaussmeter might improve measurement accuracy of the Hall effect.

Lastly, even though graphene is currently a high-interest material, it costs approximately $\$ 110$ per $250 \mathrm{~mL}$ [41]. Further, graphene is hard to manage since folding the atom-thick layer onto itself results in graphite. Due to its nanoparticle characteristics in dry form it can cause health issues [42]. Even though the idea of printing graphene sensors was initially explored [43], special inkjet printers can only achieve the feat of printing this material. For these reasons, carbon ink sensors, being a much cheaper alternative, should still be explored. Furthermore, a novel Hall sensor geometry might enhance the magnetic sensitivity of the carbon ink. 


\section{Semiconductor Hall Sensor}

The traditional method for fabricating Hall sensors involves the use of semiconductor devices. Most Hall sensors, are fabricated from an n-doped region surrounded by a p-doped region, as per the diagram below, in Figure 4-1. This thesis project started with the goal to build semiconductor Hall devices to test against printed sensors, but due to time constrains and measurement issues with the printed sensor, it was decided to continue working on the printed devices instead. Nonetheless, some progress on the semiconductor devices was made.

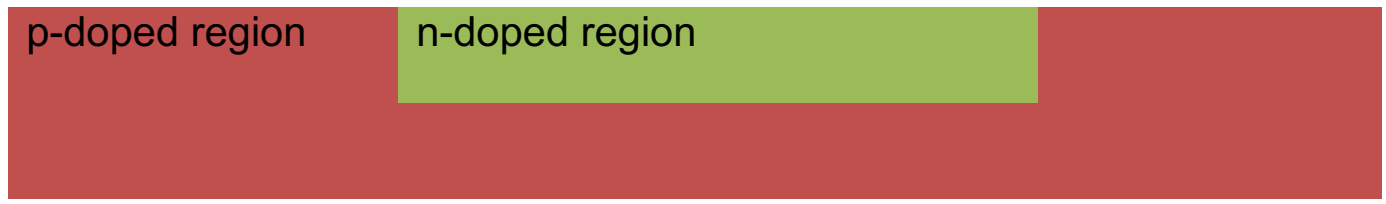

Figure 4-1. Cross-section of Hall sensor device in silicon chip.

\subsection{Motivation and Current Progress}

Even though there exists plenty of semiconductor-based Hall sensors, the ability to design and fabricate new geometries allows to make a direct comparison between printed and semiconductor sensors, ultimately improving understanding of magnetic field sensitivity, fabrication imperfections of Hall devices and feasibility of printed sensors. With this in mind, semiconductor-based sensors were designed parallel to the printed ones for performance comparison.

First, using AutoCAD, the desired sensors were designed and grouped as an individual chip. The chip was then replicated throughout the $100 \mathrm{~mm}$ silicon wafer to ensure the most amount of chips can be obtained from the wafer. Each of the 10 chips that can fit in the wafer consist of 8 different Hall devices, as seen in 
Figure 4-2. The designed Hall sensors feature the following geometries: large and small traditional crosses with and without center holes, squares with contacts on the edge of the device and contacts offset in the center of the device, and an octagon with a hole and one without. The dimensions of the sensors can be found in Figure 4-3.

The first mask containing all chips serves as a diffusion mask, that prevents dopant diffusion wherever the mask is dark, and thus prevents light from activating the positive photoresist. To facilitate access to the sensors, a simple diffusion mask will not suffice. Masks for the contact pads and vias are required. This makes a total of 3 masks for the wafer. The masks, layered together in Figure 4-4, were printed at $8,000 \mathrm{dpi}$ thanks to Dr. Keif. 


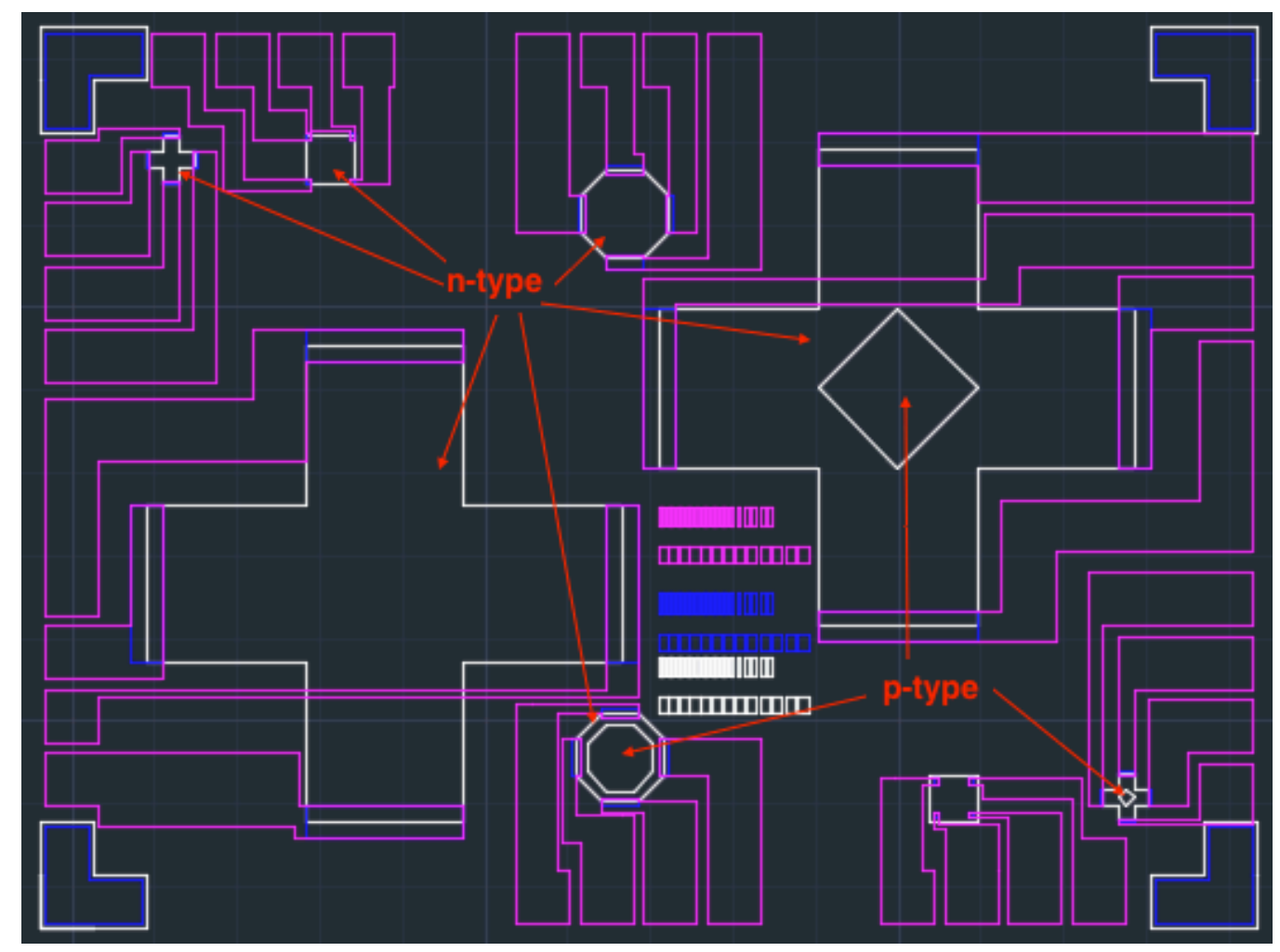

Figure 4-2. Individual chip with 8 distinct Hall devices. Mask \#1 (white) is used for dopant diffusion. Mask \#2 (blue) helps setting the metal contacts, and Mask \#3 (magenta) connects the metal contacts to vias towards the end of each chip for ease of access to each sensor. $\mathrm{N}$ - and $\mathrm{p}$-type refer to the doping polarity in that specific region.

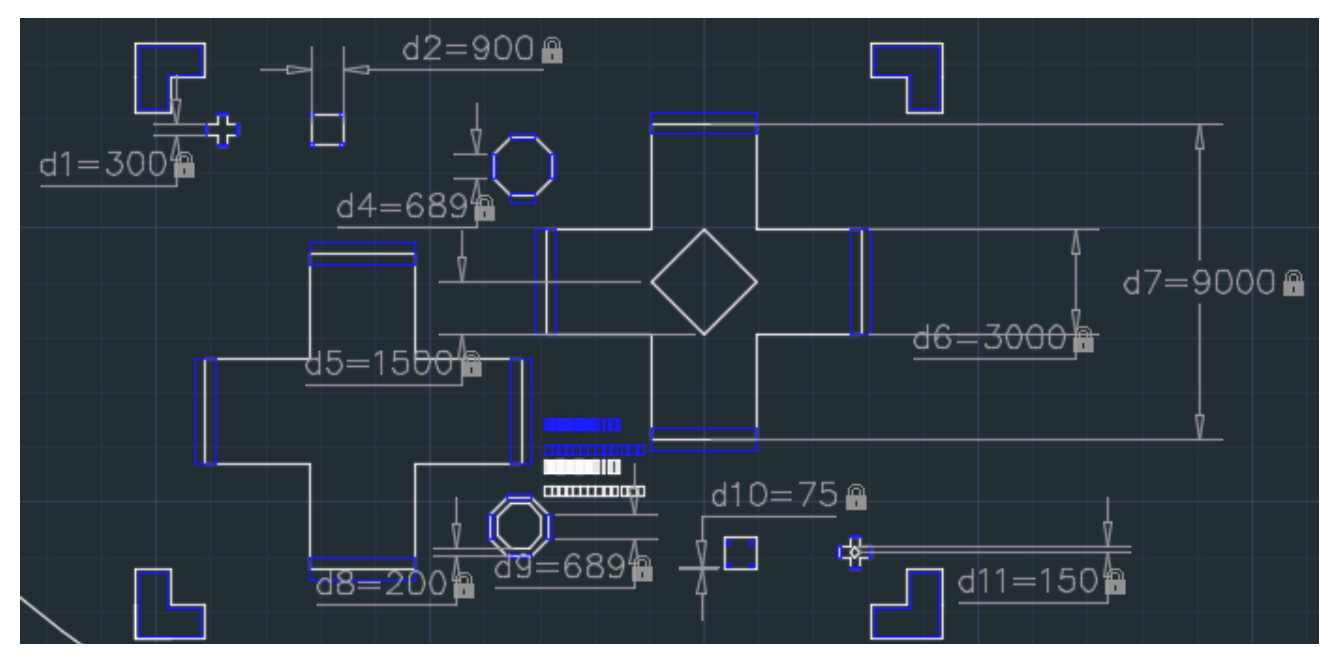

Figure 4-3. Dimensions of all designed Hall devices. d8 denotes the separation between the edge of the octagon and its hole. $\mathrm{d} 10$ denotes the horizontal and vertical separation between the sensor and its contact pads. d11 denotes the size of the internal square. 


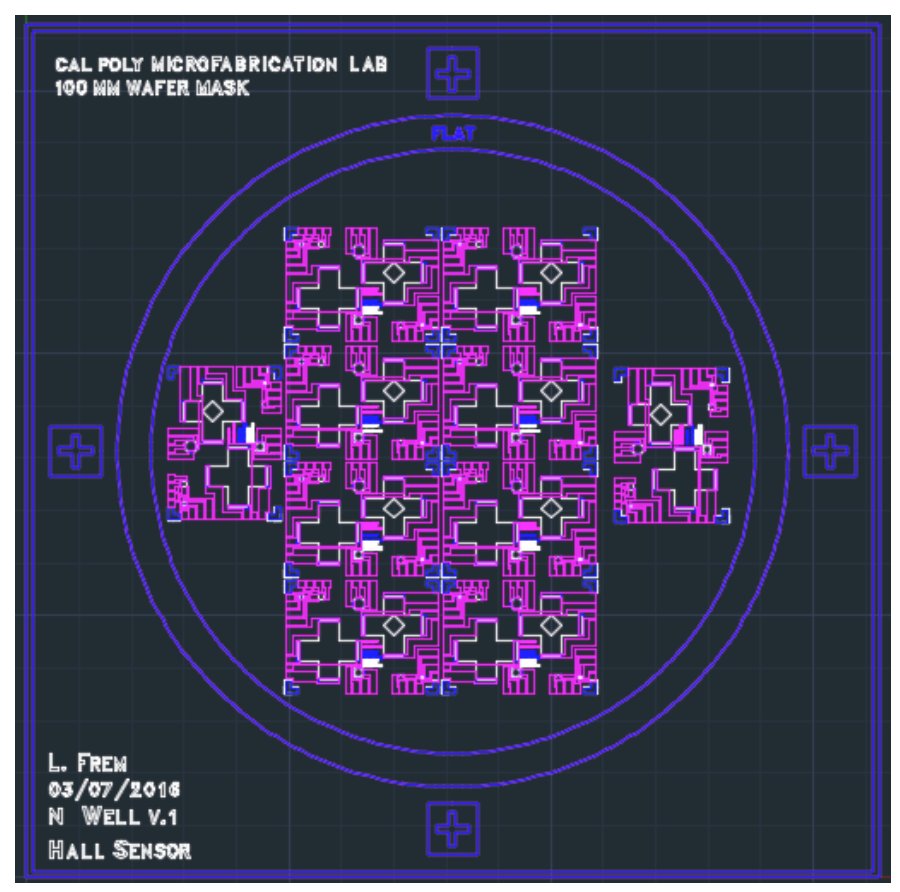

Figure 4-4. Layered exposure masks for a single wafer. Several Hall devices were designed to test performance of different geometries.

\subsection{Future Work}

The sensors were designed to test the effectiveness of novel Hall geometries. However, before fabricating any of the sensors with new geometries, these must first be simulated to test their effectiveness as Hall devices. Any sensor validated by the simulation should then be fabricated to test its performance. The Appendix presents basic procedures to fabricate any desired sensors. Furthermore, once the effective sensors are fabricated, data from testing these can further validate the magnetoconductivity and Gaussian conductivity models. 


\section{Summary of Results}

This chapter summarizes all findings throughout this project. First, the use of COMSOL was validating by replicating Sun and Kosel's Hall voltage results by sweeping a magnetic field from -5 to $+5 \mathrm{~T}$, as seen from the next two figures. This match indicates that we can use COMSOL to model Hall effect sensors, and hence proceed to model SJM's Hall sensor.

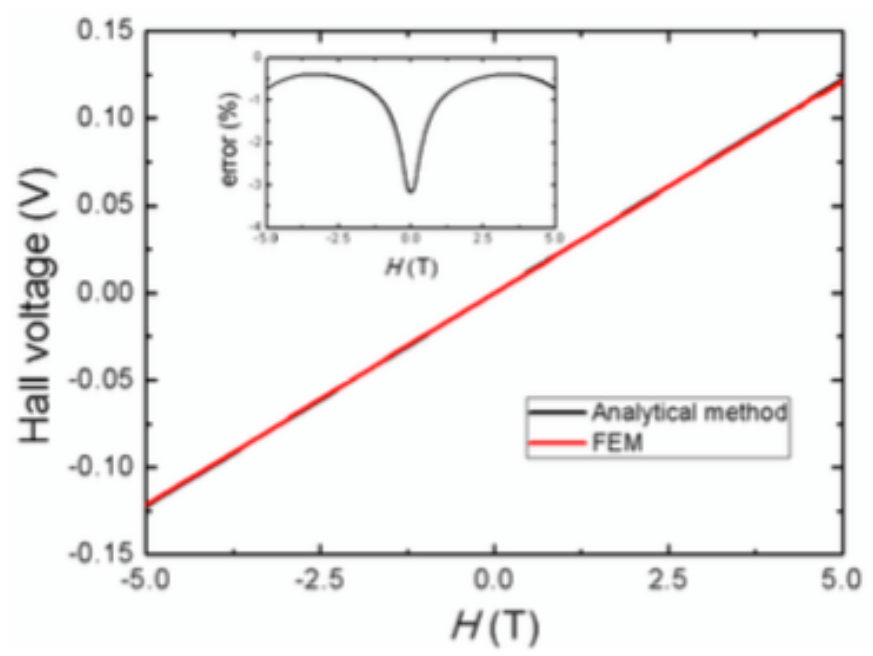

Figure 5-1. Sun and Kosel's Hall voltage as a function of the magnetic field calculated analytically and by FEM. The inset shows the error between the results from these two methods [7]. 


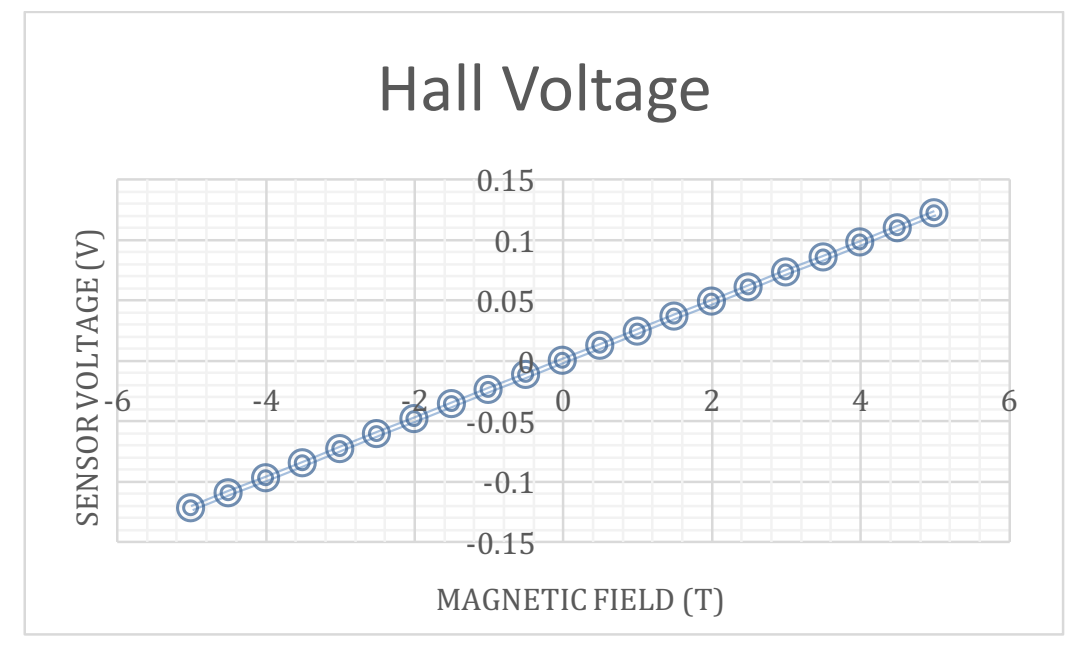

Figure 5-2. Hall voltage obtained via COMSOL FEM simulation.

After inputting sensor material parameters as reported by SJM and sweeping these values (Figure 5-3 and Figure 5-4), the material properties that matched the sensors' behavior were chosen. Furthermore, the model was enhanced to replicate voltage offset non-idealities by using the Gaussian conductivity model. A Monte Carlo analysis was performed on this model with a population of 250 samples. The results from a $15 \%$ standard deviation of the Gaussian conductivity were the ones that aligned the closest to SJM's Hall sensors' population offset and are plotted in Figure 5-5 and Figure 5-6, and Table 5-1. 


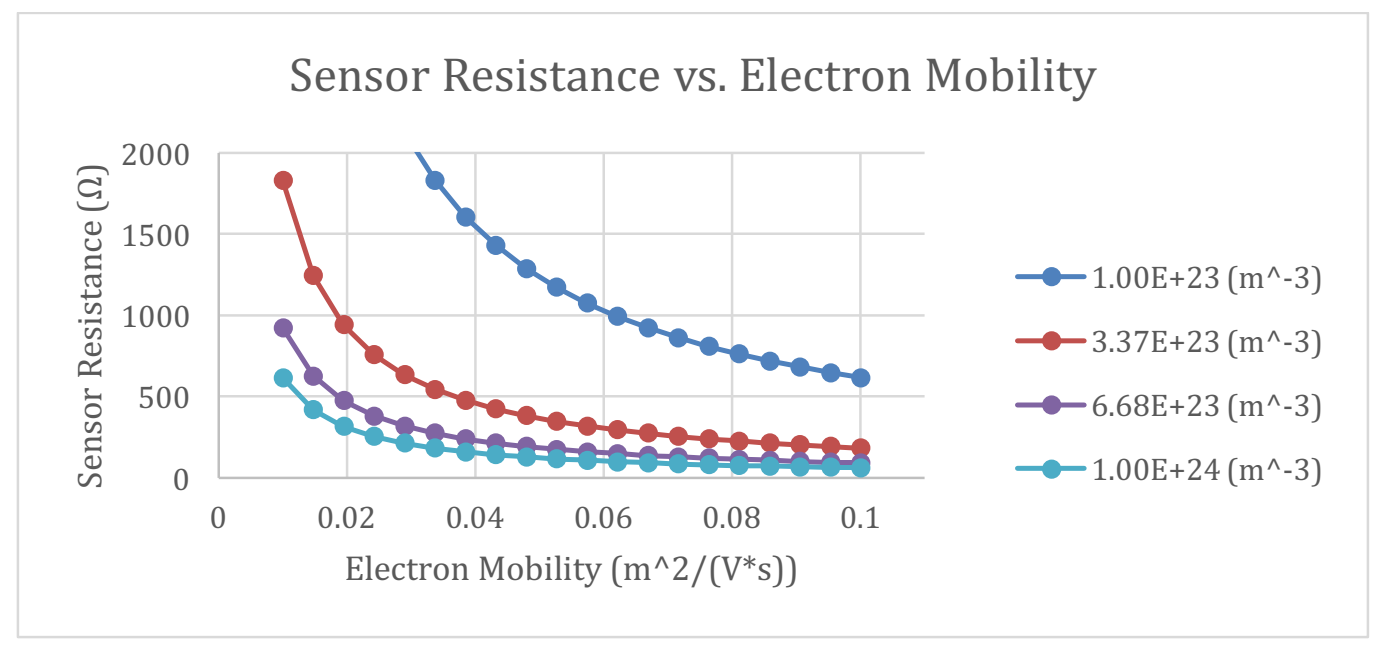

Figure 5-3. Sensor resistance vs. electron mobility with different electron density values.

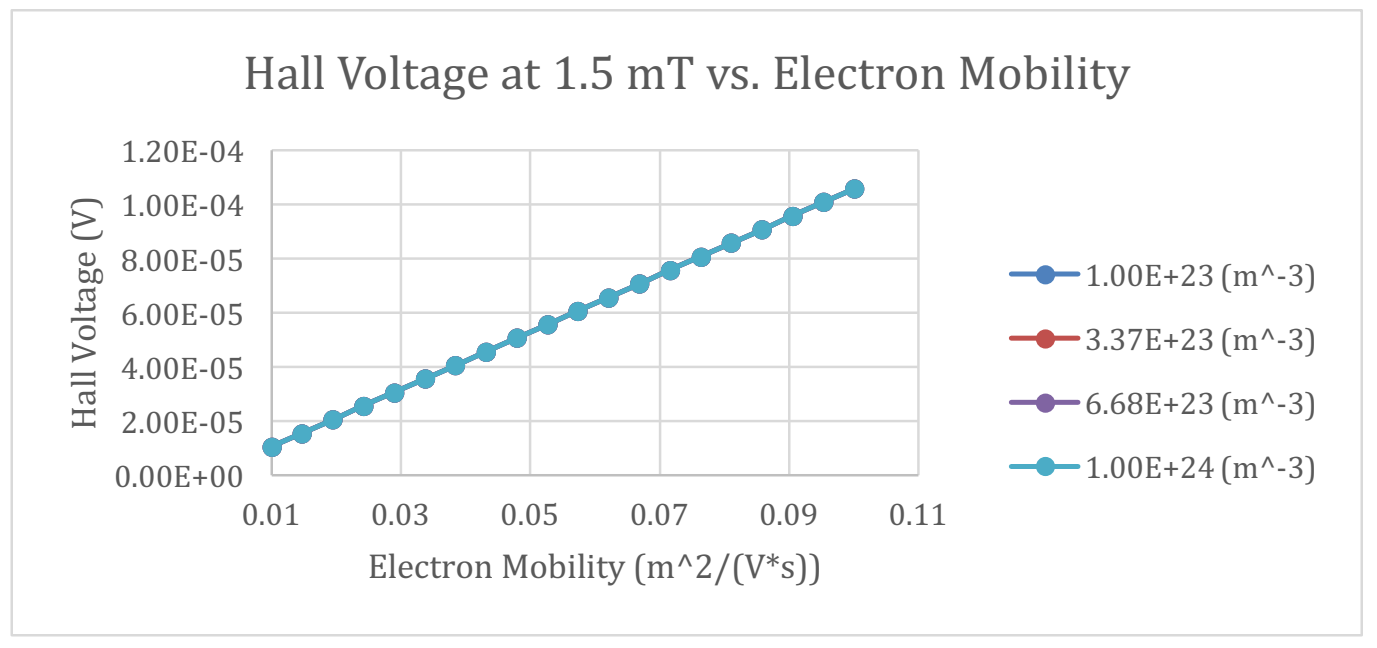

Figure 5-4. Sensor Hall voltage at constant 1.5T magnetic field vs. electron mobility with different electron density values. Target value is reached with electron mobility value of $0.0432 \mathrm{~m}^{2} /(\mathrm{V} \mathrm{s})$. Note that all electron density lines overlap, hence proving that this magnetoconductivity model disregards electron density for Hall voltage calculation. 


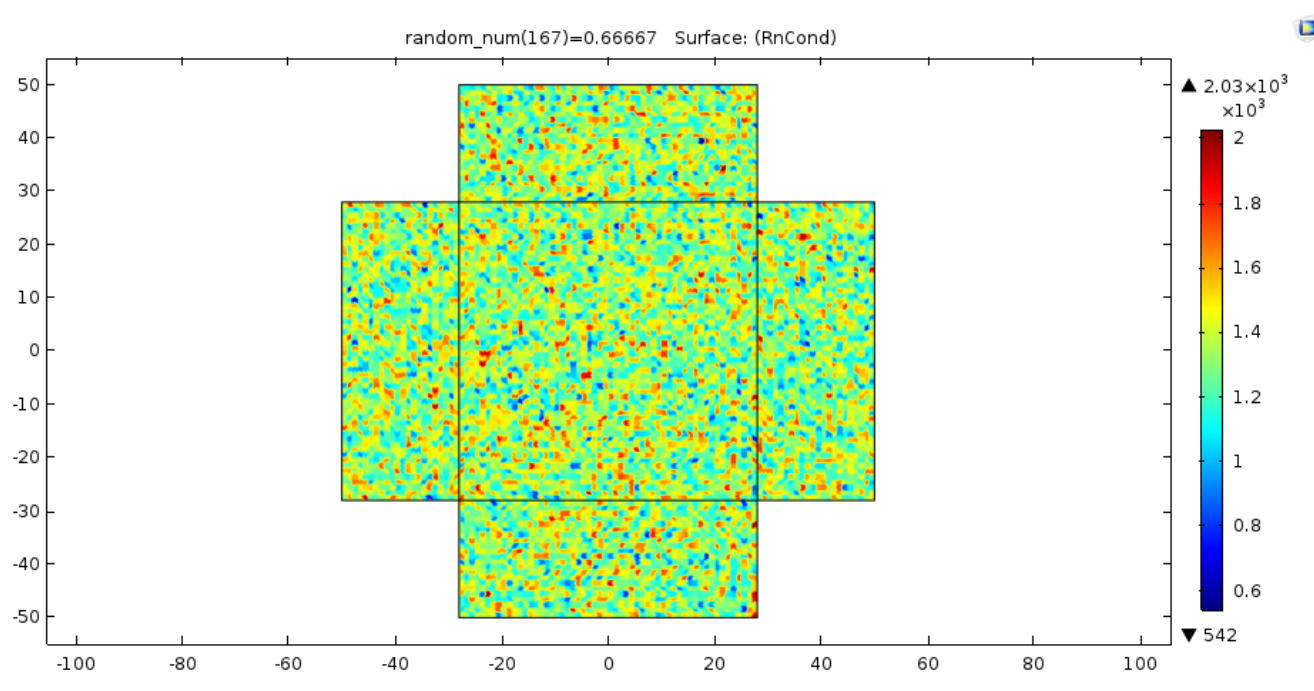

Figure 5-5. Example of conductivity model affected by discretized ion implantation imperfections. random_num(167) $=0.66667$ denotes the random seed used. A different seed produces a different conductivity distribution from the one seen above. The color bar on the right represents the conductivity value in $\mathrm{S} / \mathrm{m}$.

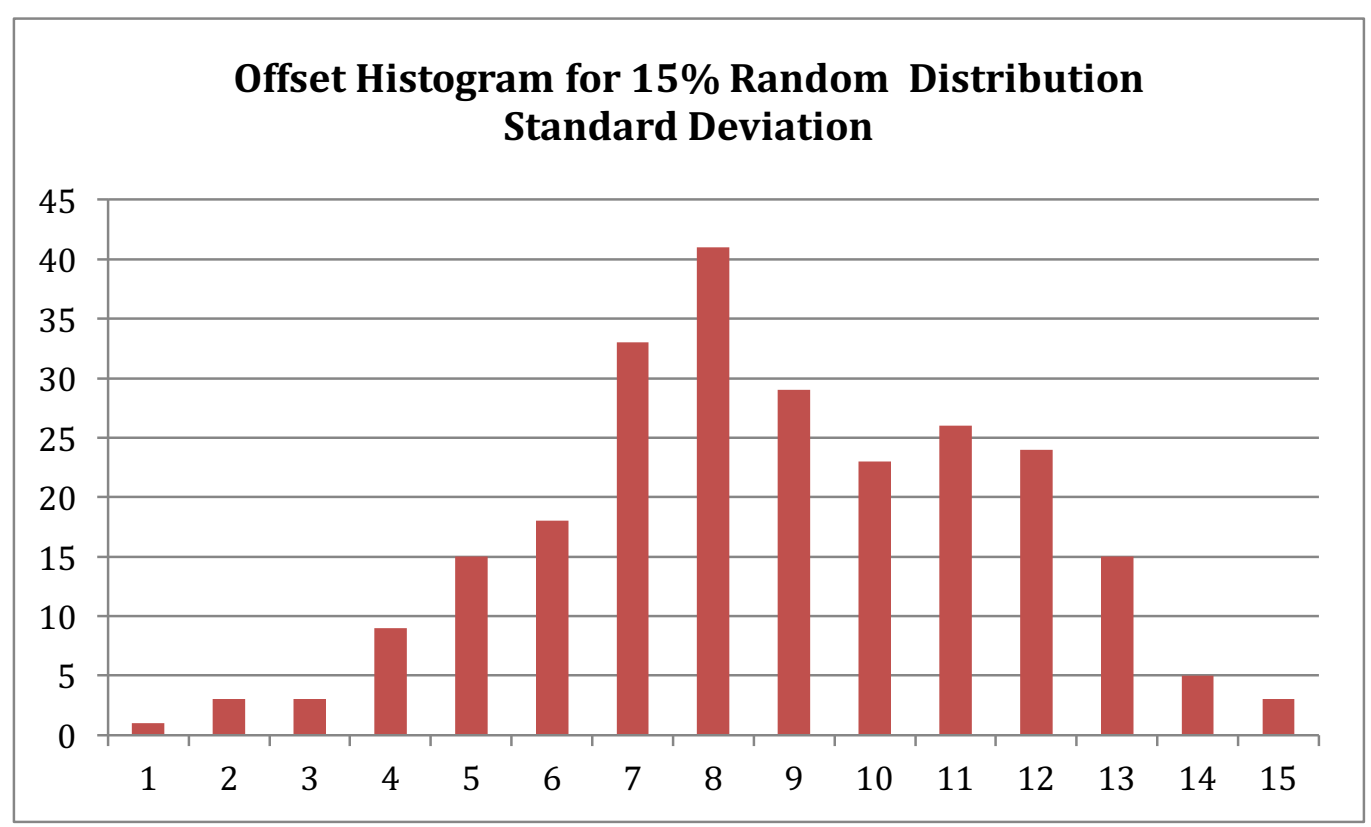

Figure 5-6. Histogram distribution for sensor offset values. The numbers in the x-axis denote bin numbers as per Table 2-2. 
Table 5-1. Histogram bin frequency distribution with respective offset values.

\begin{tabular}{|c|c|c|}
\hline \multicolumn{3}{|c|}{ Offset } \\
\hline Bin \# & Bins (V) & Frequency \\
\hline 1 & -0.00432 & 1 \\
\hline 2 & -0.0037 & 3 \\
\hline 3 & -0.00307 & 3 \\
\hline 4 & -0.00244 & 9 \\
\hline 5 & -0.00182 & 15 \\
\hline 6 & -0.00119 & 18 \\
\hline 7 & -0.00056 & 33 \\
\hline 8 & 6.47E-05 & 41 \\
\hline 9 & 0.000692 & 29 \\
\hline 10 & 0.001318 & 23 \\
\hline 11 & 0.001945 & 26 \\
\hline 12 & 0.002572 & 24 \\
\hline 13 & 0.003199 & 15 \\
\hline 14 & 0.003826 & 5 \\
\hline 15 & 0.004453 & 3 \\
\hline & More & 2 \\
\hline
\end{tabular}

Van der Pauw measurements were performed on a carbon-ink sensors, and the results are summarized in Table 5-2. The printed sensor that showed a definite magnetic response, as seen in Figure 5-7, was the $9 \mathrm{~mm}$ sensor with silver contacts. This voltage response to magnetic fields was unexpected as the research performed did not show anything close to these results. Although one should remain skeptical to these results, the carbon-ink based sensors might still be a possibility in the future. 
Table 5-2. Comparison of Van der Pauw method results from using the Helmholtz coil pair and the neodymium magnets with silver contacts on the sensors. $15 \mu \mathrm{m}$ is the assumed PET sensor thickness.

\begin{tabular}{|c|c|c|c|c|}
\hline Property & $\begin{array}{c}\text { Estimated } \\
\text { Value }\end{array}$ & $\begin{array}{c}\text { Helmholtz } \\
\text { Results }\end{array}$ & $\begin{array}{c}\text { Neodymium/Ag } \\
\text { Results }\end{array}$ & Unit \\
\hline Hole mobility & $15 \times 10^{3}$ & 8644 & 8.86 & $\frac{\mathrm{cm}^{2}}{\mathrm{Vc}}$ \\
\hline $\begin{array}{c}\text { Hole } \\
\text { concentration }\end{array}$ & $5 \times 10^{18}$ & $3.82 \times 10^{17}$ & $4.47 \times 10^{18}$ & $\mathrm{~cm}^{-3}$ \\
\hline $\begin{array}{c}\text { Sheet } \\
\text { resistance }^{\dagger}\end{array}$ & 120 & 105 & 105 & $\Omega / s q$ \\
\hline
\end{tabular}

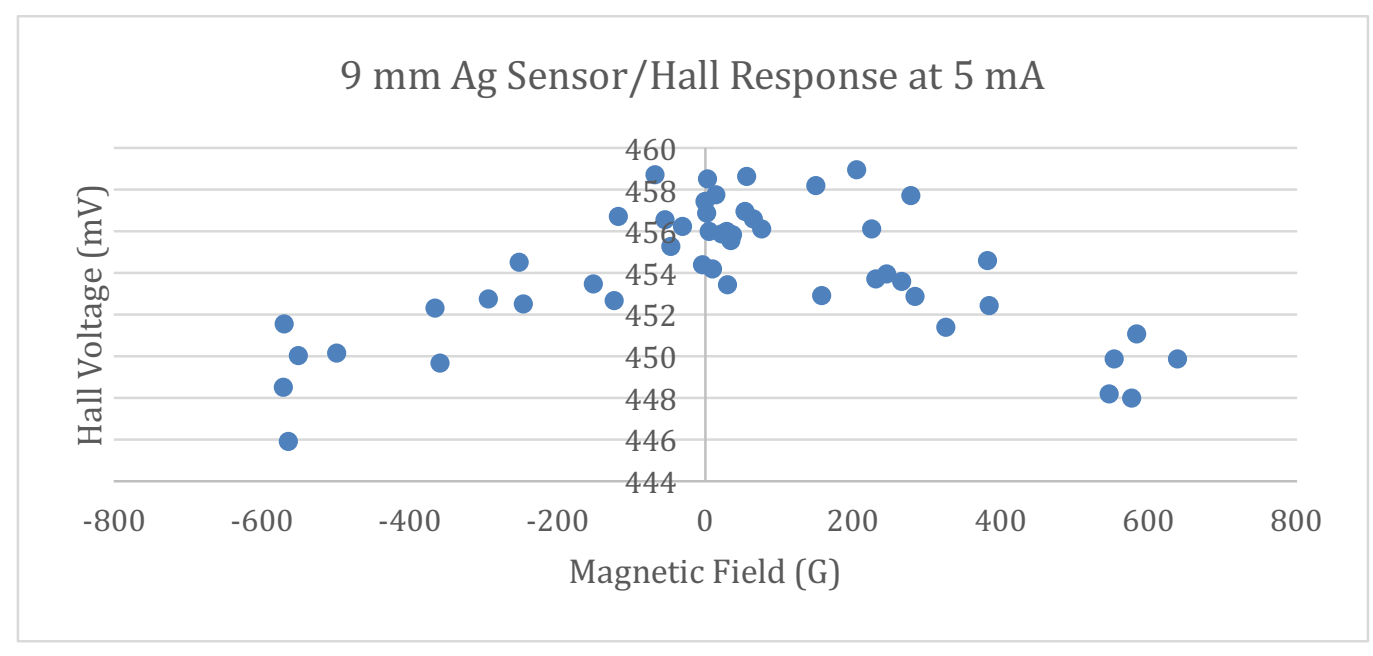

Figure 5-7. $9 \mathrm{~mm}$ sensor with silver contacts tested at $5 \mathrm{~mA}$ bias. 


\section{REFERENCES}

[1] A. Norton, "More Americans getting pacemakers," Reuters. [Online]. Available: $\quad \mathrm{http}: / / \mathrm{www}$. reuters.com/article/us-more-americans-gettingpacemakers-idUSBRE88P1LN20120926. [Accessed: 19-May-2016].

[2] "Hall Effect." [Online]. Available: http://hyperphysics.phyastr.gsu.edu/hbase/magnetic/hall.html. [Accessed: 23-May-2016].

[3] H. Blanchard, C. de Raad Iseli, and R. S. Popovic, "Compensation of the temperature-dependent offset drift of a Hall sensor," Sens. Actuators Phys., vol. 60, no. 1-3, pp. 10-13, May 1997.

[4] "Finite element method," Wikipedia, the free encyclopedia. 08-May-2016.

[5] O. de Weck and I. Y. Kim, "FEM Lecture," presented at the Engineering Design and Rapid Prototyping Lecture, Massachusetts Institute of Technology, 12-Jan-2004.

[6] "Electromagnetics Software - Computational Electromagnetics Modeling." [Online]. Available: https://www.comsol.com/acdc-module. [Accessed: 16May-2016].

[7] J. Sun and J. Kosel, "Finite-Element Modelling and Analysis of Hall Effect and Extraordinary Magnetoresistance Effect," in Finite Element Analysis - New Trends and Developments, F. Ebrahimi, Ed. InTech, 2012.

[8] H. P. Baltes and R. S. Popovic, "Integrated semiconductor magnetic field sensors," Proc. IEEE, vol. 74, no. 8, pp. 1107-1132, Aug. 1986.

[9] "Continuity equation," Wikipedia, the free encyclopedia. 29-May-2016.

[10] "Current density," Wikipedia, the free encyclopedia. 05-May-2016. 
[11] "Electric Field from Voltage." [Online]. Available: http://hyperphysics.phyastr.gsu.edu/hbase/electric/efromv.html. [Accessed: 30-May-2016].

[12] "Maxwell's equations," Wikipedia, the free encyclopedia. 28-May-2016.

[13] S. A. Campbell, Fabrication engineering at the micro- and nanoscale, Fourth edition. New York: Oxford University Press, 2013.

[14] L. Frem, "SJM's Hall Sensor Meeting Notes," SJM - Cal Poly, Online Meetings, Apr. 2016.

[15] "Ion implantation," Wikipedia, the free encyclopedia. 20-May-2016.

[16] V. Raineri, G. Galvagno, E. Rimini, S. Capizzi, A. L. Ferla, A. Carnera, and G. Feria, "Boron implants in silicon at tilt angles of $\mathrm{O}$ degrees and 7 degrees," Semicond. Sci. Technol., vol. 5, no. 10, p. 1007, 1990.

[17] "Human body temperature," Wikipedia, the free encyclopedia. 18-May-2016. [18] "Carrier Transport." [Online]. Available: http://ecee.colorado.edu/ bart/book/book/chapter2/ch2_7.htm. [Accessed: 14-May-2016].

[19] D. J. Dumin and E. C. Ross, "Temperature Dependence of the Hall Mobility and Carrier Concentration in Silicon-on-Sapphire Films." [Online]. Available: http://scitation.aip.org.ezproxy.lib.calpoly.edu/docserver/fulltext/aip/journal/ja p/41/7/1.1659376. $p d f ?$ expires=1463244710\&id=id\&accname=2119017\&che cksum=8AF44BBBEFDEEAC8760D496DF1695280. [Accessed: 14-May2016].

[20] "phonon | physics," Encyclopedia Britannica. [Online]. Available: http://www.britannica.com/science/phonon. [Accessed: 14-May-2016]. 
[21] E. Coatanéa, V. Kantola, J. Kulovesi, L. Lahti, R. Lin, and M. Zavochikova, "Printed electronics, now and future," in Bit bang - rays to the future, Helsinki, Finland: Helsinki University Print, pp. 63-102.

[22] "Printed Electronics - ALTANA AG." [Online]. Available: http://www.altana.com/innovation/printed-electronics.html. [Accessed: 21May-2016].

[23] "Printed electronics," Wikipedia, the free encyclopedia. 13-Apr-2016.

[24] "Printed, Organic \& Flexible Electronics Forecasts, Players \& Opportunities 2016-2026: IDTechEx." [Online]. Available: http://www.idtechex.com/research/reports/printed-organic-and-flexibleelectronics-forecasts-players-and-opportunities-2016-2026-000457.asp. [Accessed: 21-May-2016].

[25] C. Linder, "Density of Charge Carriers in Silver," Department of Physics and Astronomy, College of Charleston, Charleston, Apr. 2008.

[26] Gwent Group, "Curable Carbon Ink."

[27] D. Saada, "Diamond and graphite properties." [Online]. Available: http://phycomp.technion.ac.il/ david/thesis/node3.html. [Accessed: 22-May2016].

[28] K. S. Novoselov, A. K. Geim, S. V. Morozov, S. V. Dubonos, Y. Zhang, and D. Jiang, "Room-temperature electric field effect and carrier-type inversion in graphene films," ArXiv Prepr. Cond-Mat0410631, 2004. 
[29] H. O. Pierson, Handbook of carbon, graphite, diamond, and fullerenes: properties, processing, and applications. Park Ridge, N.J., U.S.A: Noyes Publications, 1993.

[30] CMS Magnetics ${ }^{\circledR}$ Powerful Neodymium Magnet $3 \times 1 / 2 \times 1 / 2$ " Grade N45 Black Epoxy Coated. .

[31] Eisco Scientific, "Eisco Scientific - PH0845HEL - Helmholtz Coils Helmholtz Coils (Each)." [Online]. Available: http://www.neobits.com/eisco_scientific_ph0845hel_helmholtz_coils_p62774 82.html?atc=gbp\&gclid=CLirl5bN8MwCFQeRfgodmcMFhw. [Accessed: 23May-2016].

[32] "Helmholtz coil," Wikipedia, the free encyclopedia. 07-May-2016.

[33] "Van der Pauw method," Wikipedia, the free encyclopedia. 23-Feb-2016.

[34] "Principles of Semiconductor Devices." [Online]. Available: http://ecee.colorado.edu/ bart/book/book/title.htm. [Accessed: 02-Jun-2016].

[35] D. J. Dumin, "Temperature Dependence of the Hall Mobility and Carrier Concentration in Silicon-on-Sapphire Films," J. Appl. Phys., vol. 41, no. 7, p. 3139, 1970.

[36] N50 1" Cube, Package of 1 Rare Earth Neodymium Magnet.

[37] "Build your own Gaussmeter." [Online]. Available: http://www.coolmagnetman.com/magmeter.htm. [Accessed: 22-May-2016]. [38] "Force between magnets," Wikipedia, the free encyclopedia. 11-Apr-2016. 
[39] H. Huang, D. Wang, and Y. Xu, "A Monolithic CMOS Magnetic Hall Sensor with High Sensitivity and Linearity Characteristics," Sensors, vol. 15, no. 10, pp. 27359-27373, Oct. 2015.

[40] Tensive Parker Labs Conductive Adhesive Gel, 50 Gram. .

[41] "The Price Of Graphene," Graphenea. [Online]. Available: http://www.graphenea.com/pages/graphene-price. [Accessed: 03-Jun-2016].

[42] R. Mertens, The Graphene Handbook (2016 edition). Lulu.com, 2016.

[43] K. R. Paton, E. Varrla, C. Backes, R. J. Smith, U. Khan, A. O’Neill, C. Boland, M. Lotya, O. M. Istrate, P. King, T. Higgins, S. Barwich, P. May, P. Puczkarski, I. Ahmed, M. Moebius, H. Pettersson, E. Long, J. Coelho, S. E. O'Brien, E. K. McGuire, B. M. Sanchez, G. S. Duesberg, N. McEvoy, T. J. Pennycook, C. Downing, A. Crossley, V. Nicolosi, and J. N. Coleman, "Scalable production of large quantities of defect-free few-layer graphene by shear exfoliation in liquids," Nat. Mater., vol. 13, no. 6, pp. 624-630, Apr. 2014.

[44] Georgia Tech, "Diffusion." [Online]. Available: http://www2.ece.gatech.edu/research/labs/vc/theory/diffusion.html. [Accessed: 01-Jun-2016].

[45] "Diffusion.book - Diffusionin siliconpdf.pdf." [Online]. Available: http://wwweng.Ibl.gov/ shuman/NEXT/MATERIALS\&COMPONENTS/Xe_damage/Diff usionin\%20siliconpdf.pdf. [Accessed: 01-Jun-2016]. 
Appendix: Semiconductor Hall Device Fabrication Procedures

The main steps to fabricate the proposed Hall sensor are outlined below. Cleaning and metrology steps were not included. Notice that these procedures are based on equipment available at Cal Poly's Microfabrication laboratory. Contact Dr. Savage or Dr. Mayer to gain access to this lab.

1. Start with a p-type silicon wafer.

\section{p-doped region}

Figure A-1. P-type silicon wafer.

2. Grow a $5,000 \AA$ silicon oxide film on the wafer by wet oxidation. This oxide film prevents dopant from penetrating into the wafer, as explained in later steps. Using Fick's first law and the ideal gas law, the Deal-Grove model [13, Ch. 4] presents the time required to grow such oxide film in a furnace adding $3,000 \AA$ to account for any issues,

$$
\begin{gathered}
t+\tau=\frac{X_{o x}^{2}}{B}+\frac{X_{o x}}{B / A} \\
t+0=\frac{(0.8 \mu m)^{2}}{0.58 \mu m^{2} / h r}+\frac{0.8 \mu m}{2.76 \mu \mathrm{m} / \mathrm{hr}}=1.39 \mathrm{hr}=84 \mathrm{~min}
\end{gathered}
$$

3. Where $t$ represents the time required to grow an oxide of thickness $X_{o x}, \tau$ is a time factor considered only when previous oxide is already present on the wafer, and is usually neglected under wet oxidation, and A and B are oxidation 
coefficients for silicon assuming $1100^{\circ} \mathrm{C}$. These coefficients were obtained from Cal Poly's Microfabrication Furnace SOP.

$\mathrm{SiO}_{2}$

p-doped region

Figure A-2. Oxide layer grown on silicon wafer.

4. Spin-on positive photoresist onto the wafer. The photoresist, wherever present, prevents etching of the oxide layer. Depending on the photoresist used, soft bake the wafer to drive off any remaining solvents.

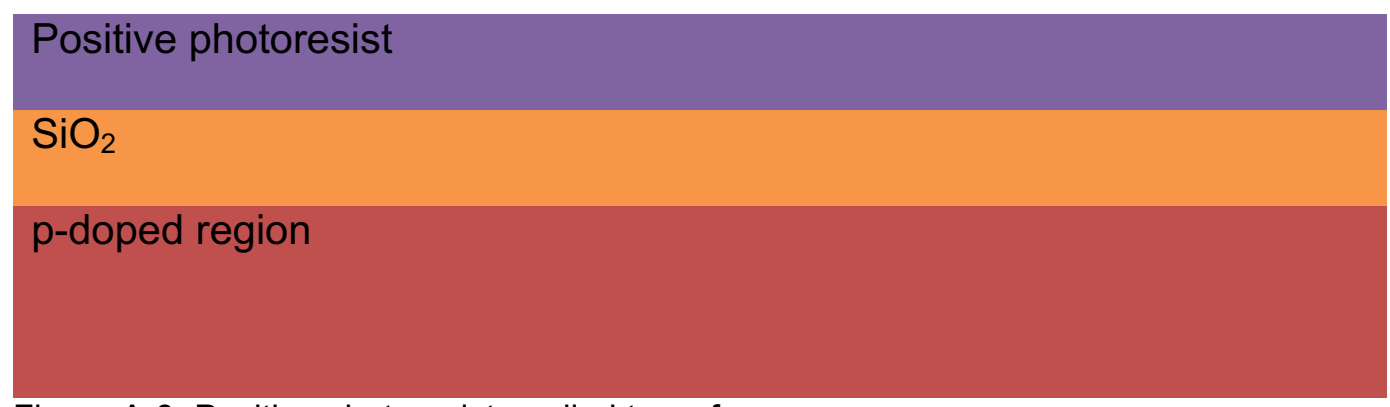

Figure A-3. Positive photoresist applied to wafer. 
5. Using the first mask, expose photoresist to UV light with a mask aligner. This makes the exposed photoresist soluble via positive photoresist developer.

UV light $\Downarrow \Downarrow \Downarrow$

\section{Mask}

Positive photoresist

$\mathrm{SiO}_{2}$

\section{p-doped region}

Figure A-4. Exposing photoresist to UV light with a mask aligner.

6. Use the developer to eliminate the exposed, and hence soluble, photoresist. This allows etching specific areas of the oxide film. Develop wafer for 4 min at $25^{\circ} \mathrm{C}$.

\section{Positive photoresist}

$\mathrm{SiO}_{2}$

\section{p-doped region}

Figure A-5. Positive photoresist developed and washed away.

7. Etch the exposed oxide film with buffered oxide etchant (BOE). Determine the time required for the etchant to reach the $p$-doped region by submerging a centimeter of a dummy wafer with an oxide film (at least $500 \AA$ thick) for one minute, and then submerging an additional centimeter of the dummy wafer. 
Repeat this procedure at least 5 times, and measure the thickness of each strip with an interferometer. The slope of the data represents the etching rate of this particular BOE solution.

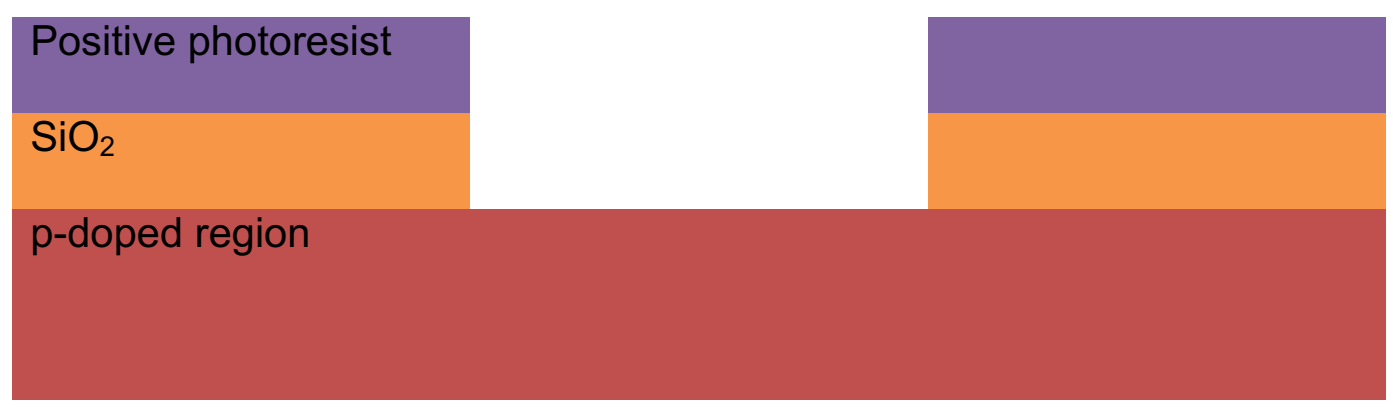

Figure A-6. Selective etching of oxide film.

8. Strip the remaining photoresist of with photoresist stripper.

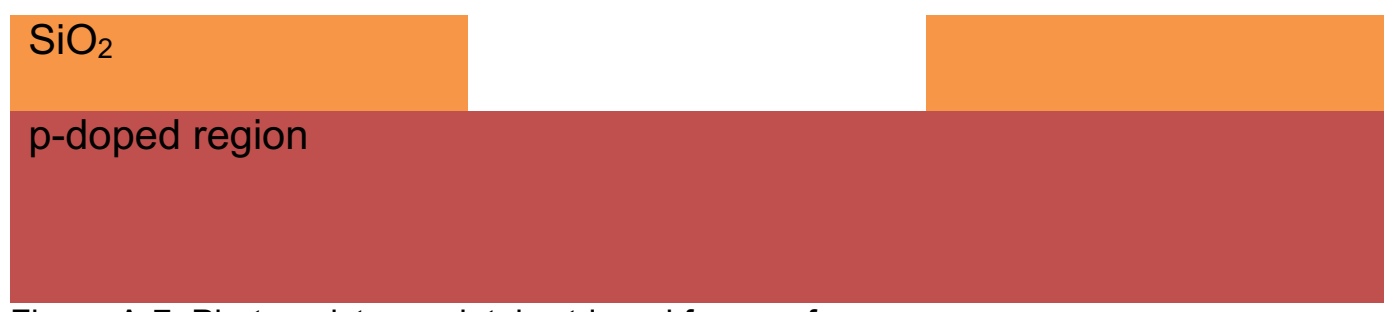

Figure A-7. Photoresist completely stripped from wafer.

9. Spin on n-type dopant onto wafer.

n-type dopant

$\mathrm{SiO}_{2}$

p-doped region

Figure A-8. n-type dopant on wafer.

10. Diffuse the dopant by placing the wafer in the furnace. The following calculations help determine time required in furnace to diffuse the dopant to a 
predefined junction depth by selecting furnace temperature. First, the neutral vacancy diffusivity [13], [44], [45] is determined by

$$
\begin{gathered}
D=D_{0} e^{-\frac{E_{a}}{k T}} \\
D=\left(10.5 \frac{\mathrm{cm}^{2}}{\mathrm{~s}}\right) e^{-\frac{3.69 \mathrm{eV}}{\left(8.617 \times 10^{-5} \mathrm{eV} / \mathrm{K}\right)(1173.15 \mathrm{~K})}}=1.474 \times 10^{-15} \frac{\mathrm{cm}^{2}}{\mathrm{~s}}
\end{gathered}
$$

Where $D_{0}$ is the diffusion coefficient for phosphorous in silicon, a temperature independent term that depends on vibrational frequency and geometry of the lattice, $E_{a}$ is the activation energy of the neutral vacancy $[13, p .48], k$ is Boltzmann's constant in $\mathrm{eV}$, and $T$ is temperature in Kelvin.

Next, assuming that a junction of $1 \mu \mathrm{m}$ is desired, the resulting junction depth $x_{j}$ for a pre-deposition diffusion [13, p. 53], [44] is calculated via

$$
x_{j}=2 \sqrt{D t} \operatorname{erfc}^{-1}\left(\frac{C_{B}}{C_{S}}\right)
$$

Solving for $t$, time in furnace in seconds,

$$
\begin{aligned}
t & =\frac{1}{D}\left(\frac{x_{j}}{2 \operatorname{erfc}^{-1}\left(\frac{C_{B}}{C_{s}}\right)}\right)^{2} \\
t & =\frac{1}{\left(1.474 \times 10^{-15} \frac{\mathrm{cm}^{2}}{\mathrm{~s}}\right)}\left(\frac{100 \times 10^{-6} \mathrm{~cm}}{2 \operatorname{erfc}^{-1}\left(\frac{1.5 \times 10^{14} \mathrm{~cm}^{-3}}{1.1 \times 10^{21} \mathrm{~cm}^{-3}}\right)}\right)^{2}=116 \mathrm{~min}
\end{aligned}
$$


In this equation, $C_{B}$ is the dopant concentration, and $C_{s}$ is the surface concentration.

n-type dopant

$\mathrm{SiO}_{2}$

p-doped region

Figure A- 9. Diffusion of n-type dopant on wafer by pre-deposition.

11. Remove dopant and oxide with hydrofluoric acid. This step finishes the Hall device on the silicon wafer. However, metal contacts are required across the edges of the sensor to reduce offset [39] in the case of the cross-shaped structures and to facilitate sensor operation and measurements.

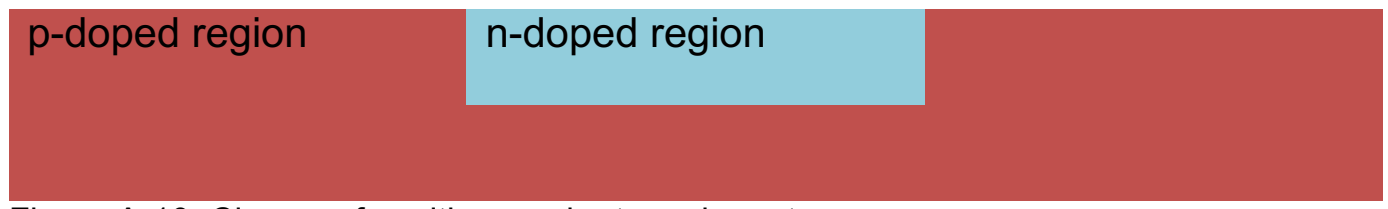

Figure A-10. Clean wafer with n- and p-type dopant.

12. Grow a $1 \mu \mathrm{m}$ oxide film.

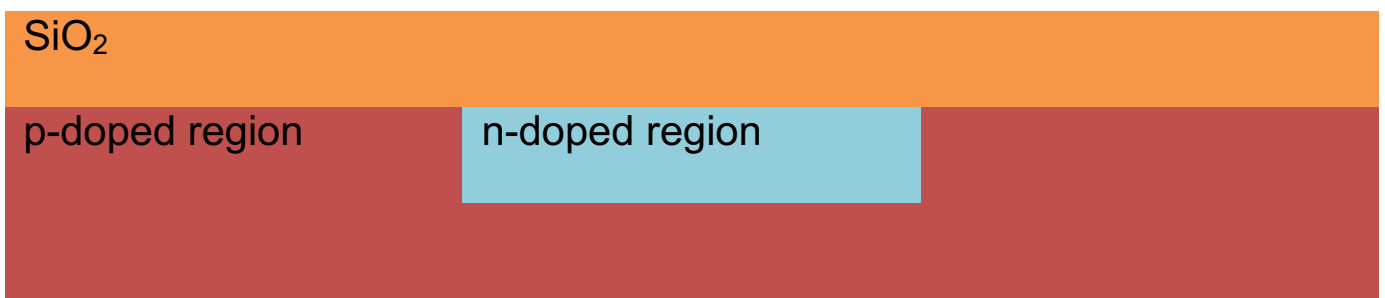

Figure A-11. Diffusion of n-type dopant on wafer by pre-deposition. 
13. Repeat lithography steps (4-8) with mask \#2.

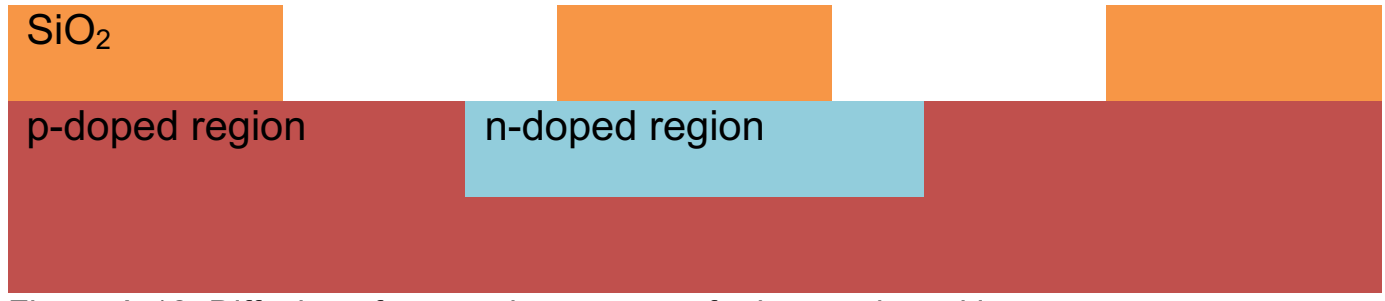

Figure A-12. Diffusion of n-type dopant on wafer by pre-deposition.

14. Sputter aluminum or any other material that might create a good contact with the sensor.

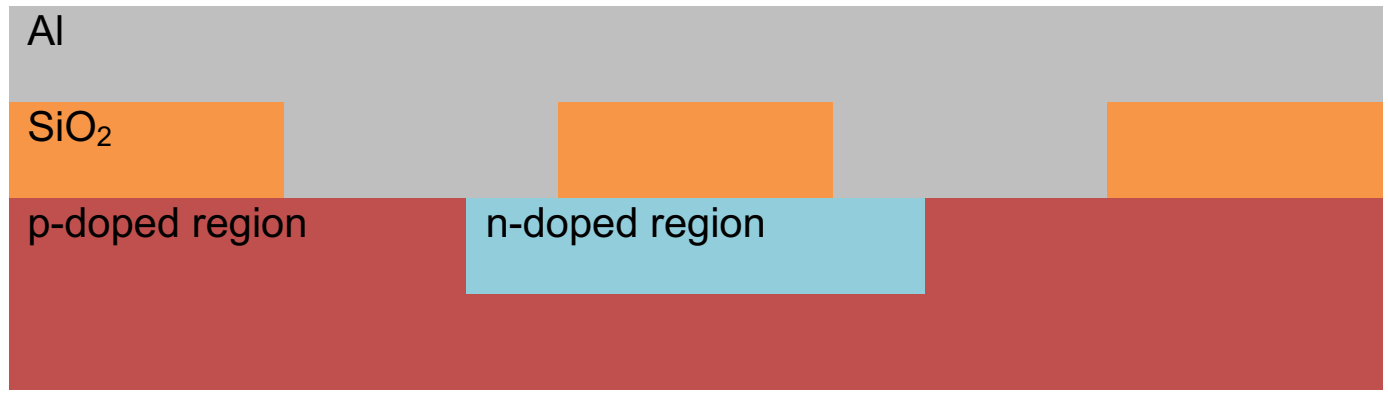

Figure A-13. Diffusion of n-type dopant on wafer by pre-deposition.

15. Repeat lithography steps (4-8) with mask \#3 and etching the aluminum.

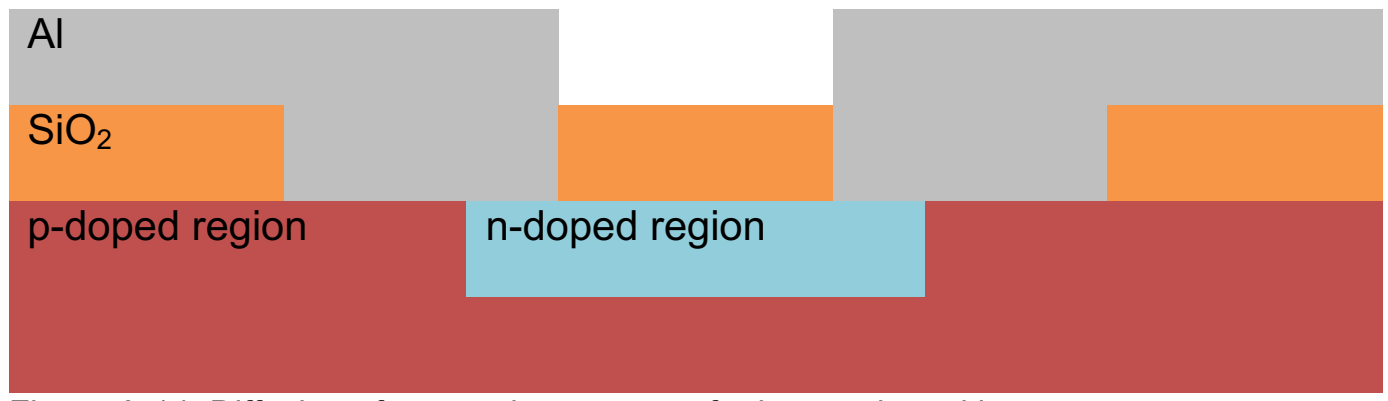

Figure A-14. Diffusion of n-type dopant on wafer by pre-deposition. 